\title{
The Cauchy Problem for Non-Linear Klein-Gordon Equations
}

\author{
Jacques C. H. Simon ${ }^{1}$ and Erik Taflin ${ }^{1,2}$ \\ 1 Département de Mathématiques, Université de Bourgogne, \\ BP 138, F-21004 Dijon Cedex, France \\ 2 Permanent address: The New Technology Division, Union des Assurances de Paris, \\ 20 ter, rue de Bezons, F-92411 Courbevoie Cedex, France
}

Received February 25, 1992; in revised form July 23, 1992

\begin{abstract}
We consider in $\mathbb{R}^{n+1}, n \geqq 2$, the non-linear Klein-Gordon equation. We prove for such an equation that there is a neighbourhood of zero in a Hilbert space of initial conditions for which the Cauchy problem has global solutions and on which there is asymptotic completeness. The inverse of the wave operator linearizes the non-linear equation. If, moreover, the equation is manifestly Poincare covariant then the non-linear representation of the Poincaré Lie algebra, associated with the non-linear Klein-Gordon equation is integrated to a non-linear representation of the Poincaré group on an invariant neighbourhood of zero in the Hilbert space. This representation is linearized by the inverse of the wave operator. The Hilbert space is, in both cases, the closure of the space of the differentiable vectors for the linear representation of the Poincare group, associated with the Klein-Gordon equation, with respect to a norm defined by the representation of the enveloping algebra.
\end{abstract}

\section{Introduction}

The problem of the existence of global solutions for the non-linear Klein-Gordon equation

$$
\left(\square+m^{2}\right) \varphi(t, x)=P\left(\varphi(t, x), \frac{\partial}{\partial t} \varphi(t, x), \nabla \varphi(t, x)\right), \quad m^{2}>0,
$$

$t \in \mathbb{R}, x \in \mathbb{R}^{n}, \varphi(t, x) \in \mathbb{C}, \nabla=\left(\partial_{1}, \ldots, \partial_{n}\right), \partial_{i}=\frac{\partial}{\partial x_{i}}, \Delta=\sum_{i=1}^{n} \partial_{i}^{2}, \square=\frac{\partial^{2}}{\partial t^{2}}-\Delta$, and

$n \geqq 1$, has been studied by various authors during the last two decades under different hypotheses on $P$ and $n$. It is difficult to give here an exhaustive description of the results already obtained and we shall only mention some of the results which, we believe, are the most significant for the case where $P$ is a $C^{\infty}$ function vanishing at zero together with its first derivatives. 
For $n=3$, the existence of global solutions was first established by Simon [6] for data given at $t=\infty$ and then by Simon, Taflin [7] for data at $t=\infty$ for coupled Klein-Gordon equations with several masses, Klainerman [4] for data at $t=0$ and Shatah [5] for data at $t=0$. The method used in the papers $[6,7]$ was that of linearization of the non-linear equation in the sense introduced in [1]. The main difficulty to solve was to establish time decrease properties for the second order term in the perturbation series of the wave operator composed with the time evolution of the linear Klein-Gordon equation. In fact, the quadratic term of the evolution group of (1.1) appears as a coboundary of the quadratic term of the wave operator. The higher order terms were then directly obtained from the YangFeldman-Källén equation (cf. [7, Eq. $\left.\left(1.1^{\prime}\right)\right]$ ) by simply using the $L^{\infty}$ estimate for free solutions and common Sobolev estimates. The construction of the wave operator gave existence of global solutions of Eq. (1.1) for small final conditions $\varphi_{0}, \dot{\varphi}_{0}$, with $\hat{\varphi}_{0}, \hat{\varphi}_{0} \in C_{0}^{\infty}\left(\mathbb{R}^{3}\right)$, where $\hat{f}$ is the Fourier transform of $f$. The method of [4] was based on a new $L^{2}-L^{\infty}$ estimate for the inhomogeneous Klein-Gordon equation which gave existence of global solutions of Eq. (1.1) for small initial conditions $\varphi_{0}, \dot{\varphi}_{0} \in C_{0}^{\infty}\left(\mathbb{R}^{3}\right)$. The result of [4] also applies to the case of systems of Klein-Gordon equations with arbitrary combinations of masses. A common drawback of references $[6,7,4]$ is that asymptotic completeness cannot be established on the sets of data that were considered. The method of article [5] was basically the same as that of [6], with in addition the use of an energy estimate.

For $n=2$, Hörmander [2] proved that the life-span $T_{\varepsilon}$ of a solution of Eq. (1.1) with initial conditions $\varphi_{0}=\varepsilon u_{0}, \dot{\varphi}_{0}=\varepsilon \dot{u}_{0}, u_{0}, \dot{u}_{0} \in C_{0}^{\infty}\left(\mathbb{R}^{3}\right), \varepsilon \geqq 0$ at $t=0$ satisfies $\varepsilon \log T_{\varepsilon} \rightarrow \infty$ as $\varepsilon \rightarrow 0$. The method in [2] is based on $L^{2}-L^{\infty}$ estimates of [4] adapted to $n=2$ and on a symbolic calculus giving approximate solutions.

In the present paper we prove that in the case $n \geqq 2$, Eq. (1.1) has global

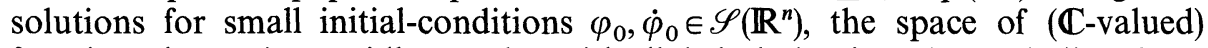
functions decreasing rapidly together with all their derivatives. As we shall see later in this introduction, it is natural, because of group theoretical reasons, to take the space $\mathscr{S}\left(\mathbb{R}^{n}\right) \oplus \mathscr{S}\left(\mathbb{R}^{n}\right)$ as the space of initial conditions for Eq. (1.1). We prove that there is a neighbourhood of zero in $\mathscr{S}\left(\mathbb{R}^{n}\right) \oplus \mathscr{S}\left(\mathbb{R}^{n}\right)$ on which we have asymptotic completeness.

To keep this article within a reasonable length, we shall impose two restrictions on $P$ :

i) $P$ is a polynomial,

ii) $P$ is covariant under Poincare transformations.

Concerning the proofs of the above result, $n=2$ represents the worst case. For this reason we only prove the results for $n=2$. However, they are valid for $n \geqq 2$ and without the restrictions i) and ii). In fact, when $n \geqq 3$ we can follow the same proof except that the norm $q_{N}$ defined by (4.10) shall contain the factor $(1+t)^{n / 2}$. When Eq. (1.1) is not Poincaré covariant the proof is still valid taking care of the fact that the equation changes under the Poincare group action. This will be discussed in the appendix. In fact, we restrict our redaction to the covariant case for purely aesthetic reasons. When $P$ is a $C^{\infty}$ function, which is not a polynomial it can be considered as the sum of a polynomial of degree 3 and a $C^{\infty}$ function with a zero of fourth order at zero. We can follow our method to obtain the scattering operator up to order 3 and then use classical methods for the rest term. In this case the scattering operator is not necessarily an analytic function of the data. 
We write Eq. (1.1) as an evolution equation by introducing the variable $a(t)$ $=\left(a_{+}(t), a_{-}(t)\right)$ :

$$
a_{\varepsilon}(t)=\dot{\varphi}(t)+\varepsilon i \omega(-i \nabla) \varphi(t), \quad \varepsilon= \pm 1,
$$

where $\omega(-i \nabla)=\left(m^{2}-\Delta\right)^{1 / 2}$ and $\dot{\varphi}(t, x)=\frac{\partial}{\partial t} \varphi(t, x)$. The inverse of transformation of (1.2) is

$$
\varphi(t)=(2 i \omega(-i \nabla))^{-1}\left(a_{+}(t)-a_{-}(t)\right), \quad \dot{\varphi}(t)=2^{-1}\left(a_{+}(t)+a_{-}(t)\right) .
$$

Equation (1.1) then reads

$$
\frac{d}{d t} a(t)=i \omega(-i \nabla)\left(a_{+}(t),-a_{-}(t)\right)+(F(a(t)), F(a(t))),
$$

where

$$
\begin{aligned}
F(a(t))= & P(\varphi(t), \dot{\varphi}(t), \nabla \varphi(t)) \\
= & P\left((2 i \omega(-i \nabla))^{-1}\left(a_{+}(t)-a_{-}(t)\right), 2^{-1}\left(a_{+}(t)+a_{-}(t)\right),\right. \\
& \left.(2 i \omega(-i \nabla))^{-1} \nabla\left(a_{+}(t)-a_{-}(t)\right)\right) .
\end{aligned}
$$

Let $\Pi=\left\{P_{\mu}, M_{\alpha \beta} \mid 0 \leqq \mu \leqq n, 0 \leqq \alpha<\beta \leqq n\right\} \quad$ be a standard basis of the Poincaré Lie algebra $\mathfrak{p}=\mathbb{R}^{n+1} \oplus \operatorname{so}(n, 1)$ in $1+n$ dimensions. $P_{0}$ is the time translation generator, $P_{i}, 1 \leqq i \leqq n$, the space translation generators, $M_{i j}, 1 \leqq i<j \leqq n$, the space rotation generators and $M_{0 j}$ the boost generators. When $n=2$ we define $R=M_{12}$ and $N_{i}=M_{0 i}, \quad i=1,2$. We define a linear representation $T^{1}$ of $\mathfrak{p}$ in $E_{\infty}=\mathscr{S}\left(\mathbb{R}^{n}\right) \oplus \mathscr{S}\left(\mathbb{R}^{n}\right)$ by:

$$
\begin{gathered}
T_{P_{0}}^{1}\left(f_{+}, f_{-}\right)=i \omega(-i \nabla)\left(f_{+},-f_{-}\right), \\
T_{P_{i}}^{1}\left(f_{+}, f_{-}\right)=\partial_{i}\left(f_{+}, f_{-}\right), \quad 1 \leqq i \leqq n, \\
T_{M_{i j}}^{1}\left(f_{+}, f_{-}\right)=m_{i j}\left(f_{+}, f_{-}\right), \quad m_{i j}=x_{i} \partial_{j}-x_{j} \partial_{i}, \\
T_{M_{0} j}^{1}\left(f_{+}, f_{-}\right)=\left(i \omega(-i \nabla) x_{j} f_{+},-i \omega(-i \nabla) x_{j} f_{-}\right), \quad 1 \leqq j \leqq n .
\end{gathered}
$$

$T^{1}$ is the differential of a continuous representation of the Poincare group $\mathscr{P}_{0}=\mathbb{R}^{n+1} \otimes S O(n, 1)$ in the space $E=L^{2}\left(\mathbb{R}^{n}, \mathbb{C}\right) \oplus L^{2}\left(\mathbb{R}^{n}, \mathbb{C}\right)$ and $E_{\infty}$ is the space of differentiable vectors for this representation (cf. [8]). Suppose given once for all an order on the set $\Pi$. Then, in the universal enveloping algebra $U(\mathfrak{p})$ of $\mathfrak{p}$, the subset $\Pi^{\prime}$ of all the products $X_{1}^{\alpha_{1}} X_{2}^{\alpha_{2}} \ldots X_{d}^{\alpha_{d}}$, where $X_{i} \in \Pi, 0 \leqq \alpha_{i}, 1 \leqq i \leqq d$ and $X_{1}<X_{2}$ $<\ldots<X_{d}$, is well known to be a basis of $U(\mathfrak{p})$. If $Y=X_{1}^{\alpha_{1}} \ldots X_{d}^{\alpha_{d}} \in \Pi^{\prime}$ we define $|Y|=|\alpha|=\sum_{1 \leqq i \leqq d} \alpha_{i}$. Let $E_{i}, i \in \mathbb{N}$ be the completion of $E_{\infty}$ with respect to the norm

$$
\|f\|_{E_{i}}=\left(\sum_{Y \in \Pi^{\prime},|Y| \leqq i}\left\|T_{Y}^{1} f\right\|_{E}^{2}\right)^{1 / 2}
$$

where $T_{Y}^{1}, Y \in U(\mathfrak{p})$ is defined by the canonical extension of $T^{1}$ to the enveloping algebra $U(\mathfrak{p})$ of $\mathfrak{p}$.

We next define the non-linear analytic representation $T$ of $\mathfrak{p}$ on $E_{\infty}$, in the sense of [1], obtained by the fact that Eq. (1.1) is manifestly covariant:

$$
T_{X}=T_{X}^{1}+\widetilde{T}_{X}, \quad X \in \mathfrak{p},
$$


where $T^{1}$ is given by (1.6) and for $f \in E_{\infty}$,

$$
\begin{gathered}
\widetilde{T}_{P_{0}}(f)=(F(f), F(f)), \\
\widetilde{T}_{P_{i}}(f)=0, \quad \widetilde{T}_{M_{i j}}(f)=0, \\
\widetilde{T}_{M_{0 j}}(f)=\left(x_{j} F(f), x_{j} F(f)\right), \quad 1 \leqq j \leqq n .
\end{gathered}
$$

The homogeneous part of $T$ of degree $l$ will be denoted by $T^{l}$.

We can extend the linear map $X \mapsto T_{X}$, from $\mathfrak{p}$ the vector space of all mappings from $E_{\infty}$ to $E_{\infty}$, to the enveloping algebra $U(\mathfrak{p})$ by defining inductively $T_{1}=I$, for 1 being the identity element in $U(\mathfrak{p}), T_{Y X}$ by

$$
T_{Y X}(f)=\left(\left(D T_{Y}\right)(f)\right)\left(T_{X}(f)\right), \text { for } Y \in U(\mathfrak{p}) \text { and } X \in \mathfrak{p} .
$$

Here $(D A)(f)$ denotes the Fréchet derivative of $A$ at $f$. In the following, when $A, B$ are differential maps we shall define $D A . B$ by $(D A . B)(f)=((D A)(f))(B(f))$. This inductive definition gives a linear map $T$ of $U(\mathfrak{p})$ into the space of polynomial operators on $E_{\infty}$. In fact, the vector field $T_{X}, X \in \mathfrak{p}$ defines a linear differential operator $\xi_{X}$ of degree at most one on the space $C^{\infty}\left(E_{\infty}\right)$, by $\xi_{X} F=D F . T_{X}$, $F \in C^{\infty}\left(E_{\infty}\right)$. The fact that $X \mapsto T_{X}$ is a non-linear representation of $p$ implies that $X \mapsto \xi_{X}$ is a linear representation of $p$ on the space of linear differential operators of degree at most one on $C^{\infty}\left(E_{\infty}\right)$. This linear continuous representation has a canonical extension $Y \mapsto \xi_{Y}$ to $U(\mathfrak{p})$ on the space of linear differential operators of arbitrary order on $C^{\infty}\left(E_{\infty}\right)$. If $\eta_{Y}, Y \in U(\mathfrak{p})$ is the part of $\xi_{Y}$ of degree not higher than one, then $Y \mapsto \eta_{Y}$ is a linear map of $U(\mathfrak{p})$ into the space of linear partial differential operators of degree at most one on $C^{\infty}\left(E_{\infty}\right)$. Let $Y \in U(\mathfrak{p})$. We write $Y=Z+a$, where $a \in \mathbb{C} \cdot \mathbf{1}$ and $Z$ has no component on $\mathbb{C} \cdot \mathbf{1}$ [relative to the natural graduation of $U(\mathfrak{p})]$. Then the previous definition of $T_{Y}$ gives $\eta_{Y} F=D F . T_{Z}+a F$, which proves that $Y \mapsto T_{Y}$ is a linear map on $U(\mathfrak{p})$.

As in (1.8) we define $\widetilde{T}_{Y}, Y \in U(\mathfrak{p})$ by

where $T_{Y}^{1}$ is the linear part of $T_{Y}$.

$$
T_{Y}=T_{Y}^{1}+\widetilde{T}_{Y},
$$

The linear map $X \mapsto \exp \left(t P_{0}\right) X \exp \left(-t P_{0}\right), t \in \mathbb{R}$ is an automorphism of $\mathfrak{p}$, leaving all the elements of the standard basis of $\mathfrak{p}$ invariant, except $M_{0 j}, j=1, \ldots, n$, for which

$$
\exp \left(t P_{0}\right) M_{0 j} \exp \left(-t P_{0}\right)=M_{0 j}+t P_{j} .
$$

For $Y \in U(\mathfrak{p})$ and $t \in \mathbb{R}$ let $Y(t) \in U(\mathfrak{p})$ be defined by

$$
Y(t)=\exp \left(t P_{0}\right) Y \exp \left(-t P_{0}\right) .
$$

If $a(t)$ is solution of (1.4) we have

$$
\frac{d}{d t} T_{Y(t)}(a(t))=T_{P_{0} Y(t)}(a(t))
$$

because $\frac{d}{d t} a(t)=T_{P_{0}}(a(t)), \frac{d}{d t} Y(t)=\left[P_{0}, Y(t)\right]$ and definition (1.10) gives:

$$
\begin{aligned}
\frac{d}{d t} T_{Y(t)}(a(t)) & =T_{\frac{d}{d t} Y(t)}(a(t))+\left(D T_{Y(t) \cdot} \cdot T_{P_{0}}\right)(a(t)) \\
& =T_{\left[P_{0}, Y(t)\right]}(a(t))+T_{Y(t) P_{0}}(a(t)) \\
& =T_{P_{0} Y(t)}(a(t)) .
\end{aligned}
$$


The evolution equation (1.4) is obtained from (1.14) with $Y=\mathbf{1}$ :

$$
\frac{d}{d t} a(t)=T_{P_{0}}(a(t))
$$

We shall now outline the method used to prove the existence of solutions for Eq. (1.4). The idea is to construct for $N$ large enough an invertible analytic map $A: \mathcal{O}_{N} \rightarrow E_{N}$, defined on a neighbourhood $\mathcal{O}_{N}$ of zero in $E_{N}$ which intertwines the linear representation $T^{1}$ and the non-linear representation $T$ of $\mathfrak{p}$ in the sense of [1], i.e.

$$
D A . T_{X}^{1}=T_{X} \circ A, \quad X \in \mathfrak{p} .
$$

The solutions of Eq. (1.4), with initial conditions $a(0)$, at $t=0$, in a sufficiently small neighbourhood $\mathcal{O}_{N}$ of zero in $E_{N}$ are then given by

$$
a(t)=A\left(V_{t} A^{-1}(a(0))\right), \quad t \geqq 0, \quad V_{t}=\exp \left(t T_{P_{0}}^{1}\right),
$$

provided that $V_{t} A^{-1}(a(0)) \in \mathcal{O}_{N}$ for $t \geqq 0$.

In this paper we have chosen $A$ to be one of the two non-linear wave operators for Eq. (1.4), namely the one which is formally defined by the Yang-FeldmanKällén equation

$$
A=I-\int_{0}^{\infty} V_{-s} \widetilde{T}_{P_{0}} \circ A \circ V_{s} d s, \quad I=\text { identity . }
$$

The main difficulty to prove the existence of a solution $A$ for (1.18) which is an injection $A: \mathcal{O}_{\infty} \rightarrow E_{\infty}$, is due to the presence of a quadratic term in $T_{P_{0}}$. We prove the existence of $A^{2}$ by using the enveloping algebra method developed in [6] namely

$$
\begin{aligned}
A^{2}= & -\left(T_{P_{0}}^{2}\left(T_{P_{0}}^{1} \otimes I+I \otimes T_{P_{0}}^{1}\right)+T_{P_{0}}^{1} T_{P_{0}}^{2}\right) \\
& \times\left(m^{2}-2 T_{P_{0}}^{1} \otimes T_{P_{0}}^{1}+2 \sum_{j=1}^{n} T_{P_{j}}^{1} \otimes T_{P_{j}}^{1}\right)^{-1},
\end{aligned}
$$

where we have used the fact that $T_{P_{j}}^{2}=0$ for $1 \leqq j \leqq n$. The operator

$$
m^{2}-2 T_{P_{0}}^{1} \otimes T_{P_{0}}^{1}+2 \sum_{j=1}^{n} T_{P_{j}}^{1} \otimes T_{P_{j}}^{1} \in L\left(E_{\infty} \hat{\otimes} E_{\infty}, E_{\infty} \hat{\otimes} E_{\infty}\right),
$$

where $\hat{\otimes}$ denotes the projective tensor product, is invertible. Using elementary facts about pseudo-differentiable operators, it is established in Theorem 3.7, that the linear map $f \mapsto A^{2}(g \otimes f)$ from $E$ to $E$ is continuous for $g \in W^{k, \infty}\left(\mathbb{R}^{n}\right) \oplus W^{k, \infty}\left(\mathbb{R}^{n}\right)^{1}$ if $k$ is sufficiently large. We then prove that $A^{2}\left(V_{t} g \otimes V_{t} f\right)$ has the following time decay properties:

$$
\begin{gathered}
\left\|A^{2}\left(V_{t} g \otimes V_{t} f\right)\right\|_{E} \leqq C_{g, f}(1+|t|)^{-n / 2}, \quad t \in \mathbb{R}, \\
\left\|A^{2}\left(V_{t} g \otimes V_{t} f\right)\right\|_{W^{0, \infty} \oplus W^{0, \infty} \leqq C_{g, f}(1+|t|)^{-n}, \quad t \in \mathbb{R} .}
\end{gathered}
$$

The higher order terms of $A$ can now be obtained directly from Eq. (1.18) by iteration, using only $L^{\infty}$ estimates for $V_{t} f$ and usual Sobolev inequalities. If we choose $N$ sufficiently large this gives, for each $a_{\infty} \in E_{N}$ sufficiently small, a solution $a(t)=A\left(V_{t} a_{\infty}\right) \in E, t \geqq 0$ of Eq. (1.4), i.e. Eq. (1.14) with $Y=1$.

${ }^{1}$ For $1 \leqq p \leqq \infty$ and $k \in \mathbb{N}$, the Sobolev space $W^{k, p}\left(\mathbb{R}^{n}\right)$ is the Banach space of functions $f: \mathbb{R}^{n} \rightarrow \mathbb{C}$, being in $L^{p}\left(\mathbb{R}^{n}\right)$ together with their first $k$ derivatives 
The next step consists to apply the above method to Eq. (1.14) with $Y \in U(\mathfrak{p})$. This implies that $T_{Y(t)}(a(t)) \in E$ and we prove that (Theorem 2.15)

$$
\|a(0)\|_{E_{N}} \leqq F_{N}\left(\sum_{Y \in \Pi^{\prime},|Y| \leqq N}\left\|T_{Y}(a(0))\right\|_{E}^{2}\right)^{1 / 2}
$$

where $F_{N}$ is a positive function bounded by a polynomial, and $a_{\infty}$ is sufficiently small in $E_{N}$. We can now conclude that $A\left(\mathcal{O}_{N}\right) \subset E_{N}$ if $N$ is sufficiently large, and that $A$ has a local inverse on a neighbourhood $\mathcal{O}_{N}^{\prime}$ of zero in $E_{N}$. This shows that Eq. (1.4) has a solution $t \mapsto a(t), t \geqq 0$ for each $a(0) \in \mathcal{O}_{N}^{\prime}$ and that $\lim _{t \rightarrow \infty}\left(V_{-t} a(t)\right)=a_{\infty} \in \mathcal{O}_{N}$. Instead of considering Eq. (1.18), we could have considered the corresponding equation for the scattering problem at $t=-\infty$, so we can conclude that there is a neighbourhood $\mathcal{O}_{N}^{0}$ of zero in $E_{N}$ such that Eq. (1.4) has a solution $t \mapsto a(t), t \in \mathbb{R}$ for each $a(0) \in \mathcal{O}_{N}^{0}$. In addition $\mathcal{O}_{N}^{0}$ can be chosen such that there are neighbourhoods $\mathcal{O}_{N}^{ \pm}$of zero in $E_{N}$ and analytic wave operators $\Omega_{ \pm}$(here $\Omega_{+}=A$ ) with the following property (asymptotic completeness): $\Omega_{\varepsilon}: \mathcal{O}_{N}^{\varepsilon} \rightarrow \mathcal{O}_{N}^{0}$ is an analytic bijection.

Finally, we state in this paragraph the main results of this article. If $\mathcal{O}\left(\right.$ resp. $\left.\mathcal{O}^{\prime}\right)$ is an open neighbourhood in a Banach space $B$ (resp. $\left.B^{\prime}\right)$, let $\mathscr{H}\left(\mathcal{O}, \mathcal{O}^{\prime}\right)$ denotes the space of analytic functions from $\mathcal{O}$ to $\mathcal{O}^{\prime}$, endowed with the topology of uniform convergence on closed bounded subsets of $\mathcal{O}$.

Theorem 1.1. For $n \geqq 2$ there exists $N_{0} \geqq 0$ and a neighbourhood $\mathcal{O}_{N_{0}}^{0}$ of zero in $E_{N_{0}}$ such that, if $\mathcal{O}_{N}^{0}=E_{N} \cap \mathcal{O}_{N_{0}}^{0}$ for $N \geqq N_{0}$ and $\mathcal{O}_{\infty}^{0}=E_{\infty} \cap \mathcal{O}_{N_{0}}^{0}$, then:

i) $T$ defined by (1.8) is a non-linear analytic Lie-algebra representation on $E_{\infty}^{0}$. For $X \in \mathfrak{p}$ and $N \geqq N_{0}, T_{X}: E_{N+1} \rightarrow E_{N}$ and $\widetilde{T}_{X}: E_{N} \rightarrow E_{N}$ are analytic maps.

ii) $T$ is the differential of a unique global non-linear analytic representation $U$ of $\mathscr{P}_{0}$, i.e. $U_{g}(\theta) \in \mathcal{O}_{N_{0}}^{0}$ for $g \in \mathscr{P}_{0}, \theta \in \mathcal{O}_{N_{0}}^{0}$ and the map $g \mapsto U_{g^{-1}}^{1} U_{g}$ is continuous from $\mathscr{P}_{0}$ into the space $\mathscr{H}\left(\mathcal{O}_{N_{0}}^{0}, E_{N_{0}}\right)$, where $U^{1}$ is the linear part of $U$.

iii) For $N \geqq N_{0}$, the map $g \mapsto U_{g-1}^{1} U_{g}$ is continuous from $\mathscr{P}_{0}$ into the space $\mathscr{H}\left(\mathcal{O}_{N}^{0}, E_{N}\right)$.

iv) $\mathcal{O}_{\infty}^{0}$ is the set of differentiable vectors of $U$.

The representation $U$ of $\mathscr{P}_{0}$ has, according to the next theorem, at least two invertible linearization operators $\Omega_{+}^{-1}$ and $\Omega_{-}^{-1}$, where $\Omega_{+}$and $\Omega_{-}$are the two wave operators for the evolution equation (1.15).

Theorem 1.2. With the notation of Theorem 1.1, $N_{0}$ can be chosen such that there exists two analytic invertible maps $\Omega_{+}: \mathcal{O}_{N_{0}}^{+} \rightarrow \mathcal{O}_{N_{0}}^{0}$ and $\Omega_{-}: \mathcal{O}_{N_{0}}^{-} \rightarrow \mathcal{O}_{N_{0}}^{0}$, where $\mathcal{O}_{N_{0}}^{+}$and $\mathcal{O}_{N_{0}}^{-}$are open neighbourhoods of zero in $E_{N_{0}}$, satisfying the following properties:

i) $U_{g} \circ \Omega_{\varepsilon}=\Omega_{\varepsilon} \circ U_{g}^{1}$, for $\varepsilon= \pm$ and $g \in \mathscr{P}_{0}$, where $U^{1}$ is the linear part of $U$.

ii) If $N \geqq N_{0}$, then $\Omega_{\varepsilon}: \mathcal{O}_{N}^{\varepsilon} \rightarrow \mathcal{O}_{N}^{0}$ is an invertible analytic map.

iii) If $h(t)=\exp \left(t P_{0}\right), t \in \mathbb{R}$ then

$$
\lim _{t \rightarrow \varepsilon \infty}\left\|U_{h(t)}\left(\Omega_{\varepsilon}(\theta)\right)-U_{h(t)}^{1} \theta\right\|_{E}=0
$$

for $\varepsilon= \pm 1$ and $\theta \in \mathcal{O}_{N_{0}}^{\varepsilon}$.

Theorem 1.1 and Theorem 1.2 give in particular the solution of the Cauchy problem at $t=0$ and solve the scattering problem for the evolution equation (1.15).

Theorem 1.3. In the situation of Theorem 1.2 the equation

$$
\frac{d}{d t} v(t)=T_{P_{0}}(v(t)), \quad v(0)=\theta \in \mathcal{O}_{N+1}^{0}, \quad N \geqq N_{0}
$$


has a unique $C^{1}$ solution $t \mapsto v(t) \in E_{N}, t \in \mathbb{R}$. Moreover,

$$
\lim _{t \rightarrow \varepsilon \infty}\left\|v(t)-V_{t} \Omega_{\varepsilon}^{-1}(\theta)\right\|_{E}=0
$$

where $V_{t}=\exp \left(t T_{P_{0}}^{1}\right)$.

Translation of the first part of Theorem 1.3 to Eq. (1.1) gives the following existence result for the non-linear Klein-Gordon equation:

Theorem 1.4. Let $P$ be a polynomial satisfying $P(0)=0, D P(0)=0$, let $n \geqq 2$ and let Eq. (1.1) be relativistic covariant. Then there are neighbourhoods $\mathcal{O}, \dot{\mathcal{O}}$ of zero in $\mathscr{S}\left(\mathbb{R}^{n}\right)$ such that for each initial conditions $\left(\varphi_{0}, \dot{\varphi}_{0}\right) \in \mathcal{O} \times \dot{\mathcal{O}}$ there is a unique solution $\varphi \in C^{\infty}\left(\mathbb{R} \times \mathbb{R}^{n}\right)$ of $E q$. (1.1) such that $\varphi(0, x)=\varphi_{0}(x)$ and $\left.\frac{\partial}{\partial t} \varphi(t, x)\right|_{t=0}=\dot{\varphi}_{0}(x)$ for $x \in \mathbb{R}^{n}$.

\section{Properties of the Non-Linear Representation}

In this paragraph we deduce estimates for $T_{Y}^{n}\left(f_{1} \otimes \ldots \otimes f_{n}\right), n \geqq 2, Y \in U(\mathfrak{p})$, and $f_{i} \in E_{\infty}$. We then deduce an explicit expression of $T_{X Y}$ for $X \in \mathfrak{p}$ and $Y \in U(\mathfrak{p})$.

Let us introduce the spaces $E_{i}^{\infty}, i \geqq 0$, as the completions of $E_{\infty}$, for the norms

and

$$
\left\|\left(f_{+}, f_{-}\right)\right\|_{E_{0}^{\infty}}=\left\|f_{+}\right\|_{L^{\infty}\left(\mathbb{R}^{2}\right)}+\left\|f_{-}\right\|_{L^{\infty}\left(\mathbb{R}^{2}\right)}
$$

$$
\|f\|_{E_{i}^{\infty}}=\sum_{Y \in \Pi^{\prime},|Y| \leqq i}\left\|T_{Y}^{1} f\right\|_{E_{0}^{\infty}}, \quad i \geqq 1 .
$$

We introduce the notation $E^{\infty}=E_{0}^{\infty}$. Occasionally, we shall use in this paragraph the notation

$$
B_{1}=\partial_{1}(\omega(-i \nabla))^{-1}, \quad B_{2}=\partial_{2}(\omega(-i \nabla))^{-1}, \quad B_{3}=(\omega(-i \nabla))^{-1}, \quad B_{4}=I .
$$

We have for $N \geqq 0$ and $j=1,2,3,4$ :

$$
\begin{aligned}
&\left\|B_{j} f\right\|_{E_{N}} \leqq C_{N}\|f\|_{E_{N}}, \\
&\left\|B_{j} f\right\|_{E_{N}^{\infty}} \leqq C_{N}\left(\sum_{i=1,2}\left\|\partial_{i} f\right\|_{E_{N}^{\infty}}+\|f\|_{E_{N}^{\infty}}\right) \leqq C_{N}^{\prime}\|f\|_{E_{N+1}^{\infty}} .
\end{aligned}
$$

Here we have used the fact that $\left\|(\omega(-i \nabla))^{-1} g\right\|_{L^{\infty}} \leqq C\|g\|_{L^{\infty}}$. By commuting the elements in the standard basis $\Pi$ of $\mathfrak{p}$, we obtain for $f=\left(f_{+}, f_{-}\right) \in E_{\infty}$ and $N \geqq 0$ :

$$
\begin{aligned}
& \|f\|_{E_{N}} \leqq C_{N}\left(\sum_{\substack{|\alpha| \leqq N \\
|\beta| \leqq N}}\left\|x^{\alpha} \partial^{\beta} f\right\|_{E}^{2}\right)^{1 / 2} \leqq C_{N}^{\prime}\|f\|_{E_{N}}, \\
& \|f\|_{E_{N}^{\infty} \leqq C_{n}} \sum_{\substack{|\alpha| \leqq N \\
|\beta| \leqq N+1}}\left\|x^{\alpha} \partial^{\beta} f\right\|_{E^{\infty}} \leqq C_{N}^{\prime}\|f\|_{E_{N+2}^{\infty}+2} .
\end{aligned}
$$

For later reference, we also note that

$$
\sum_{|\alpha| \leqq L}\left\|\partial^{\alpha} f\right\|_{E_{N}} \leqq C_{N, L}\left\|(1-\Delta)^{L / 2} f\right\|_{E_{N}} \leqq C_{N, L}^{\prime} \sum_{|\alpha| \leqq L}\left\|\partial^{\alpha} f\right\|_{E_{N}} .
$$

We denote the set

$$
\left\{(\alpha, \beta, i, \varepsilon)\left|\alpha, \beta \in \mathbb{N}^{2}, i \in\{1,2,3,4\}^{n}, \varepsilon \in\{-1,+1\}^{n},\right| \alpha|\leqq N,| \beta \mid \leqq N^{\prime}\right\},
$$

by $D\left(N, N^{\prime}, n\right)$, where $N, N^{\prime}, n \in \mathbb{N}$. 
Lemma 2.1. Let $N \geqq 0, n \geqq 2$, and $f_{1}, \ldots, f_{n} \in E_{\infty}$. Then

$$
\left\|T_{P_{0}}^{n}\left(f_{1} \otimes \ldots \otimes f_{n}\right)\right\|_{E_{N}} \leqq C_{N, n} \sum_{D(N, N, n)}\left\|x^{\alpha} \partial^{\beta}\left(\left(B_{i_{1}} f_{1, \varepsilon_{1}}\right) \ldots\left(B_{i_{n}} f_{n, \varepsilon_{n}}\right)\right)\right\|_{L^{2}\left(\mathbb{R}^{2}\right)}
$$

and

$$
\begin{aligned}
& \left\|T_{N_{j}}^{n}\left(f_{1} \otimes \ldots \otimes f_{n}\right)\right\|_{E_{N}} \\
& \quad \leqq C_{N, n} \sum_{D(N+1, N, n)}\left\|x^{\alpha} \partial^{\beta}\left(\left(B_{i_{1}} f_{1, \varepsilon_{1}}\right) \ldots\left(B_{i_{n}} f_{n, \varepsilon_{n}}\right)\right)\right\|_{L^{2}\left(\mathbb{R}^{2}\right)}, \quad j=1,2 .
\end{aligned}
$$

Proof. It follows from definition (1.9a) of $T_{P_{0}}^{n}, n \geqq 2$ that each component of $T_{P_{0}}^{n}\left(f_{1} \otimes \ldots \otimes f_{n}\right)$ is a sum of terms $C_{i, \varepsilon}\left(B_{i_{1}} f_{1, \varepsilon_{1}}\right) \ldots\left(B_{i_{n}} f_{n, \varepsilon_{n}}\right)$, where $C_{i, \varepsilon} \in \mathbb{C}$. The first of the inequalities (2.4) applied to each of these terms gives inequality (2.7). Inequality (2.8) follows in a similar way from definition (1.9c) of $T_{N_{j}}^{n}$.

We have similar inequalities for the $E_{N}^{\infty}$ estimates of $T^{n}$. As the proof is almost the same and simple, we only state the result for later reference.

Lemma 2.2. Let $N \geqq 0, n \geqq 2$, and $f_{1}, \ldots, f_{n} \in E_{\infty}$. Then

$$
\left\|T_{P_{0}}^{n}\left(f_{1} \otimes \ldots \otimes f_{n}\right)\right\|_{E_{N}^{\infty}} \leqq C_{N, n} \sum_{D(N, N+1, n)}\left\|x^{\alpha} \partial^{\beta}\left(\left(B_{i_{1}} f_{1, \varepsilon_{1}}\right) \ldots\left(B_{i_{n}} f_{n, \varepsilon_{n}}\right)\right)\right\|_{L^{\infty}\left(\mathbb{R}^{2}\right)}
$$

and

$$
\begin{aligned}
& \left\|T_{N_{j}}^{n}\left(f_{1} \otimes \ldots \otimes f_{n}\right)\right\|_{E_{N}^{\infty}} \\
& \quad \leqq C_{N, n} \sum_{D(N+1, N+1, n)}\left\|x^{\alpha} \partial^{\beta}\left(\left(B_{i_{1}} f_{1, \varepsilon_{1}}\right) \ldots\left(B_{i_{n}} f_{n, \varepsilon_{n}}\right)\right)\right\|_{L^{\infty}\left(\mathbb{R}^{2}\right)}, \quad j=1,2 .
\end{aligned}
$$

As $T_{X}, X \in \mathfrak{p}$ is a polynomial from $E_{\infty}$ to $E_{\infty}$, Proposition 10 of [1] and the next theorem show that $T$ is the differential of a unique analytic representation of the Poincaré group.

Theorem 2.3. If $n \geqq 2, N \geqq 2$, and $X$ is an element of the standard basis $\Pi$ of $\mathfrak{p}$, then

$$
\left\|T_{X}^{n}(f)\right\|_{E_{N}} \leqq C_{N, n}\|f\|_{E_{N}}^{n} .
$$

Proof. According to the definition of $T_{X}^{n}$ and Lemma 2.1,

$$
\left\|T_{X}^{n}(f)\right\|_{E_{N}} \leqq C_{N, n} \sum_{D(N+1, N, n)}\left\|x^{\alpha} \partial^{\beta}\left(\left(B_{i_{1}} f_{\varepsilon_{1}}\right) \ldots\left(B_{i_{n}} f_{\varepsilon_{n}}\right)\right)\right\|_{L^{2}\left(\mathbb{R}^{2}\right)} .
$$

Leibnitz formula for $\partial^{\beta}$ on a product implies that the terms inside the summation sign are bounded by

$$
I(\alpha, \beta, i)=C_{N, n}^{\prime} \sum_{\beta_{1}+\ldots+\beta_{n}=\beta}\left\|x^{\alpha}\left(\partial^{\beta_{1}} B_{i_{1}} f_{\varepsilon_{1}}\right) \ldots\left(\partial^{\beta_{n}} B_{i_{n}} f_{\varepsilon_{n}}\right)\right\|_{L^{2}\left(\mathbb{R}^{2}\right)} .
$$

Introduce

$$
J\left(\alpha, \beta_{1}, \ldots, \beta_{n}, i\right)=\left\|x^{\alpha}\left(\partial^{\beta_{1}} B_{i_{1}} f_{\varepsilon_{1}}\right) \ldots\left(\partial^{\beta_{n}} B_{i_{n}} f_{\varepsilon_{n}}\right)\right\|_{L^{2}\left(\mathbb{R}^{2}\right)} .
$$

Let $N=2$. If $|\beta|=\left|\beta_{1}\right|+\ldots+\left|\beta_{n}\right|=2$ and $\left|\beta_{j}\right|=2$ for some $1 \leqq j \leqq n$, then $\beta_{l}=0$ for $l \neq j$ and (using $\left.\|g\|_{L^{\infty}\left(\mathbb{R}^{2}\right)} \leqq C\|g\|_{W^{2,2}\left(\mathbb{R}^{2}\right)}\right)$

$$
J\left(\alpha, \beta_{1}, \ldots, \beta_{n}, i\right) \leqq C_{n}\left\|x^{\alpha_{j}}\left(\partial^{\beta_{j}} B_{i_{j}} f_{\varepsilon_{j}}\right)\right\|_{L^{2}} \prod_{l \neq j}\left\|x^{\alpha_{l}} f_{\varepsilon_{l}}\right\|_{W^{2,2}},
$$

where $\alpha_{1}+\ldots+\alpha_{n}=\alpha$. We choose $\alpha_{1}, \ldots, \alpha_{n}$ such that $\left|\alpha_{j}\right| \leqq 2$ and $\left|\alpha_{l}\right| \leqq 1$, which is possible as $\left|\alpha_{1}\right|+\ldots+\left|\alpha_{n}\right| \leqq 3$. Then $J\left(\alpha, \beta_{1}, \ldots, \beta_{n}, i\right) \leqq C_{n}\|f\|_{E_{2}}^{n}$, where we have used (2.3) and (2.4). If $|\beta| \leqq 2$ and $\left|\beta_{l}\right| \leqq 1$ for $1 \leqq l \leqq n$, then there exist $r, s$ such that $\left|\beta_{l}\right|=0$ 
for $l \neq r, l \neq s$. In this case

$$
J\left(\alpha, \beta_{1}, \ldots, \beta_{n}, i\right) \leqq C_{n}\left\|x^{\alpha_{r}} \partial^{\beta_{r}} B_{i_{r}} f_{\varepsilon_{r}}\right\|_{L^{4}}\left\|x^{\alpha_{s}} \partial^{\beta_{s}} B_{i_{s}} f_{\varepsilon_{s}}\right\|_{L^{4}} \prod_{\substack{l \neq r \\ l \neq s}}\left\|x^{\alpha_{l}} B_{i_{l}} f_{\varepsilon_{l}}\right\|_{W^{2,2}},
$$

where $\alpha_{1}+\ldots+\alpha_{n}=\alpha$. We choose $\alpha_{1}, \ldots, \alpha_{n}$ such that $\left|\alpha_{r}\right| \leqq 2,\left|\alpha_{s}\right| \leqq 2$, and $\left|\alpha_{l}\right|=0$ for $l \neq r, s$. The Sobolev inequality $\|g\|_{L^{4}\left(\mathbb{R}^{2}\right)} \leqq C\|g\|_{W^{1,2}\left(\mathbb{R}^{2}\right)}$ gives now $J\left(\alpha, \beta_{1}, \ldots, \beta_{n}, i\right)$ $\leqq C_{n}\|f\|_{E_{2}}^{n}$ also in this case. Thus this inequality is true for all $\alpha, \beta_{1}, \ldots, \beta_{n}$ with $|\alpha| \leqq 3$ and $\left|\beta_{1}\right|+\ldots+\left|\beta_{n}\right| \leqq 2$, which together with (2.11) and (2.12) prove that $\left\|T_{X}^{n}(f)\right\|_{E_{2}} \leqq C_{n}\|f\|_{E_{2}}^{n}$.

Let $N \geqq 3$. Then

$$
J\left(\alpha, \beta_{1}, \ldots, \beta_{n}, i\right) \leqq C_{n} \min _{\substack{1 \leqq j \leq n \\ \alpha_{1}+\ldots+\alpha_{n}=\alpha}}\left\|x^{\alpha_{j}} \partial^{\beta_{j}} B_{i_{j}} f_{\varepsilon_{j}}\right\|_{L^{2}} \prod_{l \neq j}\left\|x^{\alpha_{l}} \partial^{\beta_{l}} B_{i_{l}} f_{\varepsilon_{l}}\right\|_{W^{2,2}} .
$$

Since $N \geqq 3, n \geqq 2$, and $\left|\beta_{1}\right|+\ldots+\left|\beta_{n}\right|=|\beta| \leqq N$, we can choose $j$ in (2.13) such that $\left|\beta_{l}\right|+2 \leqq[N / 2]+2 \leqq N$ for $l \neq j$, [N/2] being the integer part of $N / 2$. We now choose $\alpha$ such that $\left|\alpha_{j}\right| \leqq N$ and $\left|\alpha_{l}\right| \leqq 1$ for $l \neq j$. Then, according to (2.4) and (2.3)

$$
\left\|x^{\alpha_{j}} \partial^{\beta_{j}} B_{i_{j}} f_{\varepsilon_{j}}\right\|_{L^{2}} \leqq C\|f\|_{E_{N}} .
$$

Commutating $x^{\alpha_{l}}$ and $1-\Delta$ for $l \neq j$ we get

$$
\left\|x^{\alpha_{l}} \partial^{\beta_{l}} B_{l_{j}} f\right\|_{W^{2,2}} \leqq C\|f\|_{E_{N}} .
$$

Inequality (2.12) now gives

$$
C_{N, n}^{\prime} \sum_{\beta_{1}+\ldots+\beta_{n}=\beta} \| x^{\alpha}\left(\partial^{\beta_{1}} B_{i_{1}} f_{\varepsilon_{1}}\right) \ldots\left(\partial^{\beta_{n}} B_{i_{n}} f_{\varepsilon_{n}}\left\|_{L^{2}\left(\mathbb{R}^{2}\right)} \leqq C_{N, n}^{\prime \prime}\right\| f \|_{E_{N}}^{n},\right.
$$

which together with inequality (2.11) proves the theorem.

We shall now make explicit the structure of $T_{X Y}$, when $X \in \mathfrak{p}$ and $Y \in U(\mathfrak{p})$. This will permit us to study Eq. (1.14).

For $L \geqq 0$ and $p \geqq 1$ a set $\mathscr{G}(L, p)$ of $p$-tuples $\eta=\left(\eta_{1}, \ldots, \eta_{p}\right)$ is defined by:

a) If $L=0$ then $\mathscr{G}(0, p)=\{(\emptyset, \ldots, \emptyset)\}$, where $\emptyset$ is the empty set.

b) If $L \geqq 1$ then

$$
\mathscr{G}(L, p)=\left\{\left(\eta_{1}, \ldots, \eta_{p}\right) \mid \eta_{i} \subset \mathbb{N}_{L}, \bigcup_{1 \leqq i \leqq p} \eta_{i}=\mathbb{N}_{L}, \eta_{i} \cap \eta_{j}=\emptyset \text { for } i \neq j\right\},
$$

where $\mathbb{N}_{L}=\{1, \ldots, L\}$.

For $q_{i}=\operatorname{card} \eta_{i} \geqq 1,1 \leqq i \leqq p$, we introduce the notation

$$
\eta_{i}=\left\{\alpha_{i, 1}, \ldots, \alpha_{i, q_{i}}\right\} \text {, where } \alpha_{i, 1}<\alpha_{i, 2}<\ldots<\alpha_{i, q_{i}} .
$$

For $L \geqq 1, X_{1}, \ldots, X_{L} \in \mathfrak{p}$ and $\eta=\left(\eta_{1}, \ldots, \eta_{p}\right) \in \mathscr{G}(L, p)$ we define $Y, Y_{1}, \ldots, Y_{p} \in U(\mathfrak{p})$ by, $Y=X_{1}, \ldots, X_{L}$,

$$
Y_{i}=X_{\alpha_{i, 1}}, \ldots, X_{\alpha_{i, q_{i}}} \text { if } \operatorname{card} \eta_{i} \geqq 1 \text { and } Y_{i}=1 \text { if } \eta_{i}=\emptyset,
$$

where 1 is the unit element in $U(\mathfrak{p})$. For $L=0$ we define $Y=1$ and $Y_{i}=1$. Let $f$ be a function of $Z_{1}, \ldots, Z_{p} \in U(\mathfrak{p})$. Then $g(\eta)=f\left(Y_{1}, \ldots, Y_{p}\right)$ defines a function of $\eta \in \mathscr{G}(L, p)$. We introduce the notation $\sum_{Y, p}^{\prime}$ by

$$
\sum_{\eta \in \mathscr{G}(L, p)} g(\eta)=\sum_{Y, p}^{\prime} f\left(Y_{1}, \ldots, Y_{p}\right)
$$


Theorem 2.4. Let $Y \in U(\mathfrak{p})$ and $X \in \mathfrak{p}$. If $Y=1$ or $Y=X_{1} X_{2}, \ldots, X_{l}, l \geqq 1$, then

$$
T_{X Y}^{n}=\sum_{\substack{1 \leqq p \\ n_{1}+\ldots+n_{p}=n}} \sum_{Y, p}^{\prime} T_{X}^{p}\left(T_{Y_{1}}^{n_{1}} \otimes \ldots \otimes T_{Y_{p}}^{n_{p}}\right) .
$$

Proof. Let $Y \in \Pi^{\prime}$. If $Y=\mathbf{1}$, then formula (2.16) is reduced to $T_{X 1}^{n}=T_{X}^{n}$. As $X \mathbf{1}=X$, (2.16) is true in this case. Suppose that formula (2.16) is true for $|Y|=L$ and let $Z \in \mathfrak{p}$. According to the definition (1.10) we then have $T_{X Y Z}=D T_{X Y} \cdot T_{Z}$, which gives, with $I_{q}=\otimes^{q} I$

$$
T_{X Y Z}^{n}=\sum_{\substack{1 \leqq k \\ 0 \leqq q \leqq n \\ 0 \leqq 1}} T_{X Y}^{k}\left(I_{q} \otimes T_{Z}^{n-k+1} \otimes I_{k-q-1}\right) .
$$

Formula (2.16) gives for $|Y|=L$ :

$$
\begin{aligned}
T_{X Y Z}^{n}= & \sum_{\substack { 1 \leqq k \leqq n \\
0 \leqq q \leqq k \\
\begin{subarray}{c}{1 \\
n_{1}+\ldots+n_{p}=k{ 1 \leqq k \leqq n \\
0 \leqq q \leqq k \\
\begin{subarray} { c } { 1 \\
n _ { 1 } + \ldots + n _ { p } = k } }\end{subarray}} \sum_{\substack{Y, p \\
n^{\prime}}} T_{X}^{p}\left(T_{Y_{1}}^{n_{1}} \otimes \ldots \otimes T_{Y_{p}}^{n_{p}}\right) \\
& \times\left(I_{q} \otimes T_{Z}^{n-k+1} \otimes I_{k-q-1}\right) .
\end{aligned}
$$

We sum over $q$ in (2.17). Then

$$
\begin{aligned}
T_{X Y Z}^{n}= & \sum_{1 \leqq k \leqq n} \sum_{\substack{1 \leqq p \leqq k \\
n_{1}+\ldots+n_{p}=k}} \sum_{Y, p}^{\prime} T_{X}^{p}\left(\left(D T_{Y_{1}}^{n_{1}} \cdot T_{Z}^{n-k+1}\right) \otimes T_{Y_{2}}^{n_{2}} \otimes \ldots \otimes T_{Y_{p}}^{n_{p}}+\ldots\right. \\
& \ldots+T_{Y_{1}}^{n_{1}} \otimes\left(D T_{Y_{2}}^{n_{2}} \cdot T_{Z}^{n-k+1}\right) \otimes \ldots \otimes T_{Y_{p}}^{n_{p}}+\ldots \\
& \vdots \\
& \left.\ldots+T_{Y_{1}}^{n_{1}} \otimes T_{Y_{2}}^{n_{2}} \otimes \ldots \otimes\left(D T_{Y_{p}}^{n_{p}} \cdot T_{Z}^{n-k+1}\right)\right) .
\end{aligned}
$$

Let

$$
C_{Y_{1}}=\sum_{1 \leqq k \leqq n} \sum_{\substack{1 \leqq p \leqq k \\ n_{1}+\ldots+n_{p}=k}} T_{X}^{p}\left(\left(D T_{Y_{1}}^{n_{1}} \cdot T_{Z}^{n-k+1}\right) \otimes T_{Y_{2}}^{n_{2}} \otimes \ldots \otimes T_{Y_{p}}^{n_{p}}\right) .
$$

We observe that $\sum_{1 \leqq k \leqq n} \sum_{1 \leqq p \leqq k}=\sum_{1 \leqq p \leqq n} \sum_{p \leqq k \leqq n}$, which gives

$$
C_{Y_{1}}=\sum_{1 \leqq p \leqq n} T_{X}^{p}\left(\sum_{p \leqq k \leqq n} \sum_{n_{1}+\ldots+n_{p}=k}\left(D T_{Y_{1}}^{n_{1}} \cdot T_{Z}^{n-k+1}\right) \otimes T_{Y_{2}}^{n_{2}} \otimes \ldots \otimes T_{Y_{p}}^{n_{p}}\right) .
$$

Let $q=n_{2}+\ldots+n_{p}$. Then

$$
\begin{aligned}
& \sum_{k=p}^{n} \sum_{n_{1}+\ldots+n_{p}=k}\left(D T_{Y_{1}}^{n_{1}} \cdot T_{Z}^{n-k+1}\right) \otimes T_{Y_{2}}^{n_{2}} \otimes \ldots \otimes T_{Y_{p}}^{n_{p}} \\
&= \sum_{k=p}^{n} \sum_{q=p-1}^{k-1}\left(D T_{Y_{1}}^{k-q} \cdot T_{Z}^{n-k+1}\right) \otimes \sum_{\substack{n_{2}+\ldots+n_{p}=q \\
n_{j} \geqq 1}} T_{Y_{2}}^{n_{2}} \otimes \ldots \otimes T_{Y_{p}}^{n_{p}} \\
&= \sum_{q=p-1}^{n-1} \sum_{k=q+1}^{n}\left(D T_{Y_{1}}^{k-q} \cdot T_{Z}^{n-k+1}\right) \otimes \sum_{n_{2}+\ldots+n_{p}=q} T_{n_{j} \geqq 1}^{n_{2}} \otimes \ldots \otimes T_{Y_{p}}^{n_{p}} \\
&= \sum_{q=p-1}^{n-1}\left(D T_{Y_{1}} \cdot T_{Z}\right)^{n-q} \otimes \sum_{n_{2}+\ldots+n_{p}=q} T_{Y_{2}}^{n_{2}} \otimes \ldots \otimes T_{Y_{p}}^{n_{p}} \\
&= \sum_{q=p-1}^{n-1} \sum_{n_{1}+\ldots+n_{p}=n} \sum_{n_{2}+\ldots+n_{p}=q} T_{Y_{1} Z}^{n-q} \otimes T_{Y_{2}}^{n_{2}} \otimes \ldots \otimes T_{Y_{p}}^{n_{p}} \\
&=
\end{aligned}
$$


This gives

$$
C_{Y_{1}}=\sum_{1 \leqq p \leqq n} \sum_{n_{1}+\ldots+n_{p}=n} T_{X}^{P}\left(T_{Y_{1} Z}^{n_{1}} \otimes T_{Y_{2}}^{n_{2}} \otimes \ldots \otimes T_{Y_{p}}^{n_{p}}\right) .
$$

This formula and the corresponding formulas for the other terms in (2.16) gives:

$$
\begin{aligned}
T_{X Y Z}^{n}= & \sum_{1 \leqq p \leqq n} \sum_{n_{1}+\ldots+n_{p}=n} \sum_{Y, p}^{\prime} T_{X}^{P}\left(T_{Y_{1} Z}^{n_{1}} \otimes T_{Y_{2}}^{n_{2}} \otimes \ldots \otimes T_{Y_{p}}^{n_{p}}\right. \\
& +T_{Y_{1}}^{n_{1}} \otimes T_{Y_{2} Z}^{n_{2}} \otimes \ldots \otimes T_{Y_{p}}^{n_{p}} \\
& \vdots \\
& \left.+T_{Y_{1}}^{n_{1}} \otimes T_{Y_{2}}^{n_{2}} \otimes \ldots \otimes T_{Y_{p} Z}^{n_{p}}\right) .
\end{aligned}
$$

Let $Y^{\prime}=Y Z$. Then $\left|Y^{\prime}\right|=L+1$. Each collection $\eta_{1}^{\prime}, \eta_{2}^{\prime}, \ldots, \eta_{p}^{\prime}$ for $Y^{\prime}$ is obtained from a collection $\eta_{1}, \eta_{2}, \ldots, \eta_{p}$ for $Y$ by defining for some $l, 1 \leqq l \leqq p$,

$$
\eta_{l}^{\prime}=\eta_{l} \cup\{L+1\} \text { and } \eta_{i}^{\prime}=\eta_{i} \text { if } i \neq l .
$$

This fact and (2.19) give

$$
T_{X Y^{\prime}}^{n}=\sum_{1 \leqq p \leqq n} \sum_{n_{1}+\ldots+n_{p}=n} \sum_{Y^{\prime}, p}^{\prime} T_{X}^{p}\left(T_{Y_{1}^{\prime}}^{n_{1}} \otimes \ldots \otimes Y_{Y_{p}^{p}}^{n_{p}}\right),
$$

which proves that (2.16) is true for $|Y|=L+1$ if it is true for $|Y|=L$. Hence by induction (2.16) is true for $|Y| \geqq 0$ as it is true for $|Y|=0$.

Corollary 2.5. Let $l \geqq 1, Y \in U(\mathfrak{p}), X \in \mathfrak{p}$, and $X_{i} \in \mathfrak{p}, 1 \leqq i \leqq p$. If $Y=\mathbf{1}$ or $Y=X_{1} X_{2}, \ldots, X_{l}$, then

$$
T_{X Y}=\sum_{p \geqq 1} \sum_{Y, p}^{\prime} T_{X}^{p}\left(T_{Y_{1}} \otimes \ldots \otimes T_{Y_{p}}\right) .
$$

Proof. According to Theorem 2.4,

$$
T_{X Y}=\sum_{n \geqq 1} \sum_{1 \leqq p \leqq n} \sum_{n_{1}+\ldots+n_{p}=n} \sum_{Y, p}^{\prime} T_{X}^{p}\left(T_{Y_{1}}^{n_{1}} \otimes \ldots \otimes T_{Y_{p}}^{n_{p}}\right)
$$

Changing the order of summation we obtain

$$
\begin{aligned}
& T_{X Y}=\sum_{p \geqq 1} \sum_{n \geqq p} \sum_{n_{1}+\ldots+n_{p}=n} \sum_{Y, p}^{\prime} T_{X}^{p}\left(T_{Y_{1}}^{n_{1}} \otimes \ldots \otimes T_{Y_{p}}^{n_{p}}\right) \\
& =\sum_{p \geqq 1} \sum_{\substack{n_{i} \geqq 1 \\
1 \leqq i \leqq p}} \sum_{Y, p}^{\prime} T_{X}^{p}\left(T_{Y_{1}}^{n_{1}} \otimes \ldots \otimes T_{Y_{p}}^{n_{p}}\right) \\
& =\sum_{p \geqq 1} \sum_{Y, p}^{\prime} T_{X}^{p}\left(T_{Y_{1}} \otimes \ldots \otimes T_{Y_{p}}\right) .
\end{aligned}
$$

Expressions (1.6a)-(1.6d), (1.9a)-(1.9c) of $T_{X}, X \in \mathfrak{p}$ and Theorem 2.4 lead to an explicit expression for $T_{Y}, Y \in U(\mathfrak{p})$, suitable for establishing estimates. For $Y=X_{1} \ldots X_{L}$, where $L \geqq 1$ and where $X_{1} \ldots X_{L} \in \Pi$, let $\mathscr{L}(Y)$ be the number of factors equal to $R$ or $N_{1}$ or $N_{2}$ in $Y$. For $Y=1$, let $\mathscr{L}(Y)=0$. For $B_{i}, 1 \leqq i \leqq 4$ defined by (2.2), let $\mathscr{B}=\left\{B_{1}, B_{2}, B_{3}, B_{4}\right\}$.

Theorem 2.6. Let $Y=X_{1} \ldots X_{L}$, where $L \geqq 1$ and $X_{1} \ldots X_{L} \in \Pi$. Let $\mathscr{P}$ be a basis of the space of differential operators on $\mathbb{R}^{2}$ with constant coefficients. If $n \geqq 1$ and $f \in E_{\infty}$, then the $\varepsilon_{0}$-th component of $T_{Y}^{n}(f)$ is:

$$
\begin{aligned}
\left(T_{Y}^{n}(f)\right)_{\varepsilon_{0}}= & \sum_{\alpha, Q, D, \varepsilon}\left(C_{0}^{(n)}(Y, \alpha, Q, D, \varepsilon)\right. \\
& \left.+C_{1}^{(n)}(Y, \alpha, Q, D, \varepsilon) i \varepsilon_{0} \omega(-i D)\right) x^{\alpha} \prod_{l=1}^{n}\left(Q_{l} D_{l} f_{\varepsilon_{l}}\right),
\end{aligned}
$$


where the sum is taken over $|\alpha| \geqq 0, \alpha \in \mathbb{N}^{2}, Q \in \mathscr{P}^{n}, D \in \mathscr{B}^{n}$, and $\varepsilon \in\{-1,1\}^{n}$. The coefficients $C_{k}^{(n)}(Y, \alpha, Q, D, \varepsilon) \in \mathbb{C}$ satisfy:

(i) Only a finite number of $C_{k}^{(n)}(Y, \alpha, Q, D, \varepsilon)$ are not equal to zero, in the sum of (2.21),

(ii) $C_{k}^{(n)}(Y, \alpha, Q, D, \varepsilon)=0$, if $\sum_{1 \leqq l \leqq n} \operatorname{deg} Q_{l}>|Y|-k-\delta_{n}$,

(iii) $C_{k}^{(n)}(Y, \alpha, Q, D, \varepsilon)=0$, if $|\alpha|>\mathscr{L}(Y)$,

where $\delta_{n}=0$ if $n=1$ and $\delta_{n}=1$ if $n \geqq 2$.

Proof. It follows from expressions (1.6a)-(1.6d) and (1.9a)-(1.9c) that the theorem is true for $M=1$. Suppose that it is true for $L \leqq L_{0}$ for some $L_{0} \geqq 1$ and let $X$ be an element of the standard basis of $\mathfrak{p}$.

Let $X=P_{j}, j=1,2$. Then formula (2.16) of Theorem 2.4 and the induction hypothesis give:

$$
\begin{aligned}
\left(T_{X Y}^{n}(f)\right)_{\varepsilon_{0}}= & \left(T_{P_{j}}^{1} T_{Y}^{n}(f)\right)_{\varepsilon_{0}} \\
= & \sum_{\alpha, P, D, \varepsilon}\left(C_{0}^{(n)}(Y, \alpha, Q, D, \varepsilon)+C_{1}^{(n)}(Y, \alpha, Q, D, \varepsilon) i \varepsilon_{0} \omega(-i \nabla)\right) \\
& \times\left(x^{\alpha} \partial_{j}+\left[\partial_{j}, x^{\alpha}\right]\right) \prod_{l=1}^{n}\left(Q_{l} D_{l} f_{\varepsilon_{l}}\right) .
\end{aligned}
$$

Let $\gamma_{1}=(1,0), \gamma_{2}=(0,1)$. Then $\left[\partial_{j}, x^{\alpha}\right]=\alpha_{j} x^{\alpha-\gamma_{j}}$. We define, for $Q^{(j)}$ $=\sum_{l}\left(Q_{1}, \ldots, Q_{l-1}, \partial_{j} Q_{l}, \ldots, Q_{n}\right)$ :

$$
\begin{aligned}
& C_{k}^{(n)}\left(P_{j} Y, \alpha, Q^{(j)}, D, \varepsilon\right) \\
& \quad=C_{k}^{(n)}(Y, \alpha, Q, D, \varepsilon)+C_{k}^{(n)}\left(Y, \alpha+\gamma_{j}, Q^{(j)}, D, \varepsilon\right)\left(\alpha_{j}+1\right)
\end{aligned}
$$

and $C_{k}^{(n)}$ is equal to zero for other values of the variables. According to the induction hypothesis, only a finite number of the coefficients $C_{k}^{(n)}\left(P_{j} Y, \alpha, Q^{(j)}, D, \varepsilon\right)$ are non-vanishing, $C_{k}^{(n)}\left(P_{j} Y, \alpha, Q^{(j)}, D, \varepsilon\right)=0$ if

$$
\sum_{1 \leqq l \leqq n} \operatorname{deg} Q_{l}^{(j)}=\sum_{1 \leqq l \leqq n} \operatorname{deg} Q_{l}+1>|Y|-k-\delta_{n}+1=\left|P_{j} Y\right|-k-\delta_{n},
$$

and $C_{k}^{(n)}\left(P_{j} Y, \alpha, Q^{(j)}, D, \varepsilon\right)=0$ if $|\alpha|>\mathscr{L}\left(P_{j} Y\right)=\mathscr{L}(Y)$. This proves that statements (i), (ii), and (iii) are true when the value of the first variable in $C_{k}^{(n)}$ is $P_{j} Y$.

Let $X=N_{j}, j=1,2$. We first consider the term $T_{N_{j}}^{1} T_{Y}^{n}$ in formula (2.16) of Theorem 2.4. For this term formula (2.21) gives:

$$
\begin{aligned}
\left(T_{N_{J}}^{1} T_{Y}^{n}(f)\right)_{\varepsilon_{0}}= & \sum_{\alpha, Q, D, \varepsilon}\left(C_{0}^{(n)}(Y, \alpha, Q, D, \varepsilon) i \varepsilon_{0} \omega(-i \nabla) x_{j} x^{\alpha} \prod_{l=1}^{n}\left(Q_{l} D_{l} f_{\varepsilon_{l}}\right)\right. \\
& \left.+C_{1}^{(n)}(Y, \alpha, Q, D, \varepsilon)\left(-x_{j}\left(m^{2}-\Delta\right)+\partial_{j}\right) x^{\alpha} \prod_{l=1}^{n}\left(Q_{l} D_{l} f_{\varepsilon_{l}}\right)\right),
\end{aligned}
$$

where $\left[\omega, x_{j}\right]=-\partial_{j} \omega^{-1}$ has been used. Since $\left[\partial_{k}, x^{\alpha}\right]=\alpha_{k} x^{\alpha-\gamma_{k}},\left[\partial_{k}^{2}, x^{\alpha}\right]$ $=\alpha_{k}\left(\alpha_{k}-1\right) x^{\alpha-2 \gamma_{k}}+\alpha_{k}^{\alpha-\gamma_{k}} \partial_{k}$ we obtain

$$
\begin{aligned}
\left(T_{N_{j}}^{1} T_{Y}^{n}(f)\right)_{\varepsilon_{0}}= & \sum_{\alpha, Q, D, \varepsilon}\left(C_{0}^{(n)}(Y, \alpha, Q, D, \varepsilon) i \varepsilon_{0} \omega(-i \nabla) x^{\alpha+\gamma_{j}} \prod_{l=1}^{n}\left(Q_{l} D_{l} f_{\varepsilon_{l}}\right)\right. \\
& +C_{1}^{(n)}(Y, \alpha, Q, D, \varepsilon)\left(x^{\alpha+\gamma_{j}}\left(-m^{2}+\Delta\right)+x^{\alpha} \partial_{j}\right. \\
& \left.+\sum_{k=1}^{2}\left(x^{\alpha-2 \gamma_{k}+\gamma_{j}} \alpha_{k}\left(a_{k}-1\right)+x^{\alpha-\gamma_{k}+\gamma_{j}} \alpha_{k} \partial_{k}\right)+x^{\alpha-\omega_{j}}\right) \\
& \left.\times \prod_{l=1}^{n}\left(Q_{l} D_{l} f_{\varepsilon_{l}}\right)\right) .
\end{aligned}
$$


We define

$$
d_{1}^{(1)}\left(1, N_{j}, Y, \alpha, Q, D, \varepsilon\right)=C_{0}^{(n)}\left(Y, \alpha-\gamma_{j}, Q, D, \varepsilon\right)
$$

and $d_{0}^{(n)}(1, X, Y, \alpha, Q, D, \varepsilon)$ by identifying the coefficients in the expression

$$
\begin{aligned}
& \sum_{Q^{\prime}}\left(C_{1}^{(n)}\left(Y, \alpha-\gamma_{j}, Q^{\prime}, D, \varepsilon\right)\left(-m^{2}+\Delta\right)+C_{1}^{(n)}\left(Y, \alpha, Q^{\prime}, D, \varepsilon\right) \partial_{j}\right. \\
& \quad+\sum_{k=1}^{2}\left(\left(\alpha_{k}+2\right)\left(\alpha_{k}+1\right) C_{1}^{(n)}\left(Y, \alpha+2 \gamma_{k}-\gamma_{j}, Q^{\prime}, D, \varepsilon\right)\right. \\
& \left.\quad+\left(\alpha_{k}+1\right) C_{1}^{(n)}\left(Y, \alpha+\gamma_{k}-\gamma_{j}, Q^{\prime}, D, \varepsilon\right) \partial_{k}\right) \\
& \left.\quad+\left(\alpha_{j}+1\right) C_{1}^{(n)}\left(Y, \alpha+\gamma_{j}, Q^{\prime}, D, \varepsilon\right)\right) \prod_{l=1}^{n}\left(Q_{l}^{\prime} g_{l}\right) \\
& =\sum_{Q} d_{0}^{(n)}\left(1, N_{j}, Y, \alpha, Q, D, \varepsilon\right) \prod_{l=1}^{n}\left(Q_{l} g_{l}\right), \quad g_{1}, \ldots, g_{n} \in \mathscr{S}\left(\mathbb{R}^{2}\right),
\end{aligned}
$$

where we have defined $C_{k}^{(n)}\left(Y, \beta, Q^{\prime}, D, \varepsilon\right)=0$ if $\beta_{i}<0$ for some $1 \leqq i \leqq n$. Expressions (2.24), (2.25), and (2.26) give:

$$
\begin{aligned}
\left(T_{N_{j}}^{1} T_{Y}^{n}(f)\right)_{\varepsilon_{0}}= & \sum_{\alpha, Q, D, \varepsilon}\left(d_{0}^{(n)}\left(1, N_{j}, Y, \alpha, Q, D, \varepsilon\right)\right. \\
& \left.+d_{1}^{(n)}\left(1, N_{j}, Y, \alpha, Q, D, \varepsilon\right) i \varepsilon_{0} \omega(-i \nabla)\right) x^{\alpha} \prod_{l=1}^{n}\left(Q_{l} D_{l} f_{\varepsilon_{l}}\right)
\end{aligned}
$$

Since there is, according to the induction hypothesis and statement (i) of the theorem, only a finite number of non-vanishing terms in the sum on the left-hand side of (2.26), there is only a finite number of coefficients $d_{0}^{(n)}\left(1, N_{j}, Y, \alpha, Q, D, \varepsilon\right)$ which are not equal to zero. By statement (ii) of the theorem it follows that $d_{0}^{(n)}\left(1, N_{j}, Y, \alpha, Q, D, \varepsilon\right)=0$ if $\sum_{1 \leqq l \leqq n} \operatorname{deg} Q_{l}>\left|N_{j} Y\right|-\delta_{n}=|Y|+1-\delta_{n}$, because the coefficient of $\left(-m^{2}+\Delta\right) \prod_{l=1}^{n}\left(Q_{l}^{\prime} g_{l}\right)$ vanishes if $\sum_{1 \leqq l \leqq n} \operatorname{deg} Q_{l}^{\prime}>|Y|-1-\delta_{n}$. If $|\alpha|-1$ $>\mathscr{L}(Y)$, then the left-hand side of $(2.26)$ is zero. Hence $d_{0}^{(n)}\left(1, N_{j}, Y, \alpha, Q, D, \varepsilon\right)=0$ if $|\alpha|>\mathscr{L}(Y)+1=\mathscr{L}\left(N_{j} Y\right)$. It follows directly from (2.25) that only a finite number of the coefficients $d_{1}^{(n)}\left(1, N_{j}, Y, \alpha, Q, D, \varepsilon\right)$ are non-zero for given $Y$ and that $d_{1}^{(n)}\left(1, N_{j}, Y, \alpha, Q, D, \varepsilon\right)=0$ if $\sum_{1 \leqq l \leqq n} \operatorname{deg} Q_{l}>|Y|-\delta_{n}=\left|N_{j} Y\right|-1-\delta_{n}$ or $|\alpha|>\mathscr{L}(Y)+1$ $=\mathscr{L}\left(N_{j} Y\right)$. To sum up, if $p=1$, and $n \geqq 1$, then

a) for only a finite number of the coefficients $d_{k}^{(n)}\left(p, N_{j}, Y, \alpha, Q, D, \varepsilon\right) \neq 0$,

b) $d_{k}^{(n)}\left(p, N_{j}, Y, \alpha, Q, D, \varepsilon\right)=0$, if $\sum_{1 \leqq l \leqq n} \operatorname{deg} Q_{l}>\left|N_{j} Y\right|-k-\delta_{n}$,

c) $d_{k}^{(n)}\left(p, N_{j}, Y, \alpha, Q, D, \varepsilon\right)=0$, if $|Y|>n \mathscr{L}\left(N_{j} Y\right)$.

We next consider the terms

$$
T_{N_{j}}^{p}\left(T_{Y_{1}}^{n_{1}} \otimes \ldots \otimes T_{Y_{p}}^{n_{p}}\right), \quad 2 \leqq p \leqq n, \quad n_{1}+\ldots+n_{p}=n,
$$

in the expression (2.16). Here $Y_{1}, \ldots, Y_{p}$ are as in Theorem 2.4. For $U \in E_{\infty}$, let $v_{0}=(2 i \omega(-\nabla i))^{-1}\left(u_{+}-u_{-}\right), v_{1}=\partial_{1} v_{0}, v_{2}=\partial_{2} v_{0}, v_{3}=2^{-1}\left(u_{+}+u_{-}\right)$. Then, according to definition $(1.9 \mathrm{c})$ of $T_{N_{j}}^{p}$,

$$
\left(T_{N_{j}}^{p}(u)\right)_{\varepsilon_{0}}=x_{j} \sum_{i} b(i) v_{i_{1}} \ldots v_{i_{n}}, \quad \varepsilon_{0}= \pm 1
$$


where $b(i) \in \mathbb{C}, i \in\{0,1,2,3\}^{n}$ and $b(i)$ is symmetric in $i_{1}, \ldots, i_{n}$. It is convenient to introduce for $Z \in U(\mathfrak{p})$ :

$$
\begin{gathered}
\left(S_{Z}(f)\right)_{0}=(2 i \omega(-i \nabla))^{-1}\left(\left(T_{Z}(f)\right)_{+}-\left(T_{Z}(f)\right)_{-}\right), \\
\left(S_{Z}(f)\right)_{1}=2^{-1}\left(\left(T_{Z}(f)\right)_{+}+\left(T_{Z}(f)\right)_{-}\right),
\end{gathered}
$$

and $S_{Z}(f)=\left(\left(S_{Z}(f)\right)_{0},\left(S_{Z}(f)\right)_{1}\right)$.

It follows from the induction hypothesis and from (2.21) that if $Z \in U(\mathfrak{p})$ is a product of elements of the standard basis of $\mathfrak{p}$ and $|Z| \leqq L_{0}$, then

$$
\left(S_{Z}^{q}(f)\right)_{0}=\sum_{\alpha, Q, D, \varepsilon} C_{1}^{q}(Z, \alpha, Q, D, \varepsilon) x^{\alpha} \prod_{l=1}^{q}\left(Q_{l} D_{l} f_{\varepsilon_{l}}\right)
$$

and

$$
\left(S_{Z}^{q}(f)\right)_{1}=\sum_{\alpha, Q, D, \varepsilon} C_{0}^{q}(Z, \alpha, Q, D, \varepsilon) x^{\alpha} \prod_{l=1}^{q}\left(Q_{l} D_{l} f_{\varepsilon_{l}}\right), \quad q \geqq 1 .
$$

As we have already proved the theorem for $T_{P_{j} Z}$, the induction hypothesis implies:

$$
\begin{aligned}
\left(\partial_{j} S_{Z}^{q}(f)\right)_{0} & =\left(S_{P \gamma_{j}, Z}^{q}(f)\right)_{0} \\
& =\sum_{\alpha, Q, D, \varepsilon} C_{1}^{(q)}\left(P^{\gamma_{j}} Z, \alpha, Q, D \varepsilon\right) x^{\alpha} \prod_{l=1}^{q}\left(Q_{l} D_{l} f_{\varepsilon_{l}}\right),
\end{aligned}
$$

where $j=1,2, \gamma_{1}=(1,0)$, and $\gamma_{2}=(0,1)$.

Let

$$
a_{0}^{(q)}=C_{1}^{(q)}, \quad a_{3}^{(q)}=C_{0}^{(q)}
$$

and

$$
a_{j}^{(q)}(Z, \alpha, Q, D, \varepsilon)=C_{1}^{(q)}\left(P^{\gamma_{j}} Z, \alpha, Q, D, \varepsilon\right), \quad j=1,2 .
$$

It is readily verified that, if $q \geqq 1$ then

$\left(\mathrm{a}^{\prime}\right)$ only a finite number of the coefficients $a_{j}^{(q)}(Z, \alpha, Q, D, \varepsilon) \neq 0$,

(b') $a_{j}^{(q)}(Z, \alpha, Q, D, \varepsilon)=0$ if $\sum_{1 \leq l \leq q} \operatorname{deg} Q_{l}>|Z|$,

(c) $a_{j}^{(q)}(Z, \alpha, Q, D, \varepsilon)=0$ if $|\alpha|>l \leq q(Z)$.

According to (2.28) we have for $p \geqq 2$ :

$$
\begin{aligned}
& \left(T_{N_{j}}^{p}\left(T_{Y_{1}}^{n_{1}}(f) \otimes \ldots \otimes T_{Y_{p}}^{n_{p}}(f)\right)\right)_{\varepsilon_{0}} \\
& =\sum x_{j} x^{\alpha^{(1)}+\ldots+\alpha^{(p)} b(i)} \prod_{k=1}^{p} a_{i_{k}}\left(Y_{k}, \alpha^{(k)}, Q^{(k)}, D^{(k)}, \varepsilon^{(k)}\right) \\
& \quad \times \prod_{l_{k}=1}^{n_{k}}\left(Q_{l_{k}}^{(k)} D_{l_{k}}^{(k)} f_{\left.\varepsilon_{l_{k}}(k)\right)},\right.
\end{aligned}
$$

where the sum is taken over $i, \alpha^{(1)}, \ldots, \alpha^{(p)}, Q^{(1)}, \ldots, Q^{(k)}, D^{(1)}, \ldots, D^{(q)}, \varepsilon^{(1)}, \ldots, \varepsilon^{(k)}$. We define for $p \geqq 2$ :

$$
d_{1}^{(n)}\left(p, N_{j}, Y, \alpha, Q, D, \varepsilon\right)=0
$$

and

$$
\begin{aligned}
& d_{0}^{(n)}\left(p, N_{j}, Y, \alpha, Q, D, \varepsilon\right) \\
& \quad=\sum_{Y, p \quad \alpha=\gamma_{j}+\alpha^{(1)}+\ldots+\alpha^{(p)}} \prod_{k=1}^{p} a_{i_{k}}\left(Y_{k}, \alpha^{(k)}, Q^{(k)}, D^{(k)}, \varepsilon^{(k)}\right),
\end{aligned}
$$


where $Q=Q^{(1)} \oplus \ldots \oplus Q^{(p)}, D=D^{(1)} \oplus \ldots \oplus D^{(p)}, \varepsilon=\left(\varepsilon_{1}^{(1)}, \ldots, \varepsilon_{n_{1}}^{(1)}, \ldots, \varepsilon_{1}^{(p)}, \ldots, \varepsilon_{n_{p}}^{(p)}\right) d_{k}^{(n)}$, $k=0,1$ so defined satisfy the properties (a), (b), and (c) for $2 \leqq p \leqq n$. This is obvious for $k=1$. Property (a) follows from ( $\left(\mathrm{a}^{\prime}\right)$ in the case $k=0$. Since

$$
\sum_{1 \leqq l \leqq n} \operatorname{deg} Q_{l}=\sum_{1 \leqq k \leqq p} \sum_{1 \leqq l \leqq n_{k}} \operatorname{deg} Q_{l}^{(k)} \quad \text { and }|Y|=\sum_{1 \leqq k \leqq p}\left|Y_{k}\right|
$$

it follows that if $\sum_{1 \leqq l \leqq n} \operatorname{deg} Q_{l}>|Y|$, there exists $q$, depending on $Y_{1}, \ldots, Y_{p}, 1 \leqq q \leqq p$ such that $\sum_{1 \leq l \leq n_{q}} \operatorname{deg} Q_{l}^{(q)}>\left|Y_{q}\right|$. But then $a_{i_{q}}\left(Y_{q}, \alpha^{(q)}, Q^{(q)}, D^{(q)}, \varepsilon^{(q)}\right)=0$, which proves that $d_{0}^{(n)}\left(p, N_{j}, Y, \alpha, Q, D, \varepsilon\right)=0$. If $p \geqq 2$ then $n \geqq 2$. Therefore, property (b) is true for $p \geqq 2$ and $k=0$. Similarly, since $|\alpha|=\sum_{1 \leqq l \leqq p}\left|\alpha_{l}\right|+1$ according to (2.36) and since $\mathscr{L}\left(N_{j} Y\right)=1+\sum_{1 \leqq l \leqq p} \mathscr{L}\left(Y_{l}\right)$, it follows that $d_{0}^{(n)}\left(p, N_{j}, Y, \alpha, Q, D, \varepsilon\right)=0$ if $|\alpha|>\mathscr{L}\left(N_{j} Y\right)$. This proves that property (c) is true $p \geqq 2$ and $k=0$. Hence the properties (a), (b), (c) are true for $n \geqq 1, p \geqq 1$. We now define, for $n \geqq 1$

$$
C_{k}^{(n)}\left(N_{j} Y, \alpha, Q, D, \varepsilon\right)=\sum_{p \geqq 1} d_{k}^{(n)}\left(p, N_{j} Y, \alpha, Q, D, \varepsilon\right) .
$$

$C_{k}^{(n)}$ so defined satisfy (2.21) by construction, with $N_{j} Y$ instead of $Y$. Since (a), (b), and (c) are true for $n \geqq 1, p \geqq 1$ it follows that $C_{k}^{(n)}$ satisfy properties (i), (ii), and (iii) of the theorem with $N_{j} Y$ instead of $Y$.

The cases $X=P_{0}$ and $X=R$ are so similar that we omit them.

This proves that the theorem is true for $L \leqq L_{0}+1$, and hence by induction for every $L \geqq 1$.

Corollary 2.7. Let $f_{1}, \ldots, f_{n} \in E_{\infty}$. Then, in the situation of Theorem 2.6

$$
\begin{aligned}
& \left(T_{Y}^{n}\left(f_{1} \otimes \ldots \otimes f_{n}\right)\right)_{\varepsilon_{0}} \\
& =\sum_{\alpha, Q, D, \varepsilon}\left(C_{0}^{(n)}(Y, \alpha, Q, D, \varepsilon)+C_{1}^{(n)}(Y, \alpha, Q, D, \varepsilon) i \varepsilon_{0} \omega(-i \nabla)\right) x^{\alpha} \\
& \quad \times \prod_{l=1}^{n}\left(Q_{l} D_{l} f_{l, \varepsilon_{l}}\right) .
\end{aligned}
$$

Differentiation in (2.21) gives this result since the coefficients are symmetric.

We now turn to the problem of proving that the linear group representation with differential $T^{1}$ and that the analytic group representation with differential $T$ do have the same differential vectors in the sense of [1] in a sufficiently small neighbourhood of zero in $E$, though $T$ is not the differential of a smooth representation.

For $N \in \mathbb{N}$, let $\alpha_{Y} \in E$ for all $Y \in U(\mathfrak{p})$ such that $Y=1$ or $Y=X_{1}, \ldots, X_{L}, 1 \leqq L \leqq N$, where $X_{1}, \ldots, X_{L} \in \Pi$. We introduce

$$
\wp_{N}(a)=\left(\sum_{|Y| \leqq N}\left\|a_{Y}\right\|_{E}^{2}\right)^{1 / 2}, \quad N \geqq 0 .
$$

We note that according to definition (1.7) of \|\|$_{E_{N}}$ we have

$$
\wp_{N}\left(T^{1}(f)\right)=\|f\|_{E_{N}}, \quad N \geqq 0 .
$$

Lemma 2.8. Let $f \in E_{\infty}, L \geqq 1$ and let $Y=X_{1}, \ldots, X_{L}$, where $X_{1}, \ldots, X_{L} \in \Pi$. If $L=1$, then

$$
\left\|\widetilde{T}_{Y}(f)\right\|_{E} \leqq C_{1}\|f\|_{E_{\mathscr{C}(Y)}}\|(1-\Delta) f\|_{E}\left(1+\|(1-\Delta) f\|_{E}\right)^{\chi_{1}} .
$$


If $L \geqq 2$ and $\mathscr{L}(Y) \leqq|Y|-1$, then

$$
\begin{aligned}
\left\|\widetilde{T}_{Y}(f)\right\|_{E} \leqq & C_{L}\left\|(1-\Delta)^{\frac{1}{2} a(y)} f\right\|_{E \mathscr{L}(Y)}\left\|(1-\Delta)^{\frac{1}{2} b(y)} f\right\|_{E} \\
& \times\left(1+\left\|(1-\Delta)^{\frac{1}{2} b(y)} f\right\|_{E}\right)^{\chi_{L}}
\end{aligned}
$$

where ( $[s]$ denoting the integer part of $s \in \mathbb{R}$ )

$$
a(Y)=|Y|-1-\mathscr{L}(Y), \quad b(Y)=\left[\frac{|Y|-1}{2}\right]+2 .
$$

The constants $C_{L}$ and $\chi_{L}, L \geqq 0$ are independent of $f$.

Proof. According to (2.21) we estimate

$$
\xi=\left\|x^{\alpha} \prod_{l=1}^{n}\left(Q_{l} D_{l} f_{\varepsilon_{l}}\right)\right\|_{L^{2}}, \quad \text { for } \sum_{1 \leqq l \leqq n} \operatorname{deg} Q_{l} \leqq|Y|-1, \quad|\alpha| \leqq \mathscr{L}(Y)
$$

and

$$
\eta=\left\|\omega(-i \nabla) x^{\alpha} \prod_{l=1}^{n}\left(Q_{l} D_{l} f_{\varepsilon_{l}}\right)\right\|_{L^{2}}, \quad \text { for } \sum_{1 \leqq l \leqq n} \operatorname{deg} Q_{l} \leqq|Y|-2, \quad|\alpha| \leqq \mathscr{L}(Y) .
$$

As $\|\omega(-i \nabla) g\|_{L^{2}} \leqq C\|g\|_{W^{1,2}}$, it follows that estimates of $\eta$ will be obtained from estimates of $\xi$. Hence we only give estimates of $\xi$. First, let $L=1$. Then $\operatorname{deg} Q_{l}=0$ for $1 \leqq l \leqq n$, so

$$
\begin{aligned}
\xi(\alpha, Q, D, \varepsilon, n) & =\left\|x^{\alpha} \prod_{l=1}^{n}\left(Q_{l} D_{l} f_{\varepsilon_{l}}\right)\right\|_{L^{2}} \\
& \leqq C_{n}\left\|x^{\alpha}\left(D_{1} f_{\varepsilon_{1}}\right)\right\|_{L^{2}} \prod_{l=2}^{n}\left\|D_{l} f_{\varepsilon_{l}}\right\|_{L^{\infty}} \\
& \leqq C_{n}\left\|x^{\alpha}\left(D_{1} f_{\varepsilon_{1}}\right)\right\|_{L^{2}}\|(1-\Delta) f\|_{E}^{n-1},
\end{aligned}
$$

where we have used $\left\|D_{l} f_{\varepsilon_{l}}\right\|_{L^{\infty}} \leqq C\left\|(1-\Delta) D_{l} f_{\varepsilon_{l}}\right\|_{L^{2}} \leqq C^{\prime}\|(1-\Delta) f\|_{E}$. It follows from (2.4) and (2.3) that

$$
\left\|x^{\alpha}\left(D_{1} f_{\varepsilon_{1}}\right)\right\|_{L^{2}} \leqq\left\|x^{\alpha}\left(D_{1} f\right)\right\|_{E} \leqq C\left\|D_{1} f\right\|_{E_{|\alpha|}} \leqq C^{\prime}\|f\|_{E_{|\alpha|}}
$$

This and (2.44) give, as $|\alpha| \leqq \mathscr{L}(Y)$ :

$$
\xi(\alpha, Q, D, \varepsilon, n) \leqq C_{n}\|f\|_{E_{\mathscr{L}(Y)}}\|(1-\Delta) f\|_{E}^{n-1},
$$

where the constant $C_{n}$ depends on $\alpha, Q, D, \varepsilon$. As already pointed out in the beginning of this proof we then also have

$$
\dot{\eta}(\alpha, Q, D, Z, n) \leqq C_{n}\|f\|_{E_{\mathscr{L}(Y)}}\|(1-\Delta) f\|_{E}^{n-1} .
$$

Let the degree of the polynomials $T_{X}, X \in \mathfrak{p}$ be bounded by $\chi_{1}+2$. It follows now, after summation in $\alpha, Q, D, \varepsilon, n$, from (2.45), (2.46), and Theorem 2.6 that

$$
\left\|\widetilde{T}_{Y}(f)\right\|_{E} \leqq C_{1}\|f\|_{E_{\mathscr{L}(Y)}}\|(1-\Delta) f\|_{E}\left(1+\|(1-\Delta) f\|_{E}\right)^{\chi_{1}}, \quad|Y|=1
$$

which proves (2.40). Secondly, let $L \geqq 2$ and let $\mathscr{L}(Y) \leqq|Y|-1$. After a permutation of $1, \ldots, n$, we have

$$
\operatorname{deg} Q_{l} \leqq \operatorname{deg} Q_{1} \leqq|Y|-1
$$


in (2.42). Since in this case $\operatorname{deg} Q_{l} \leqq\left[\frac{|Y|-1}{2}\right]$ for $2 \leqq l \leqq n$, we get from (2.42),

$$
\xi(\alpha, Q, D, \varepsilon, n) \leqq C\left\|x^{\alpha}\left(Q_{1} D_{1} f_{\varepsilon_{1}}\right)\right\|_{L^{2}} \prod_{l=2}^{n}\left\|Q_{l} D_{l} f_{\varepsilon_{l}}\right\|_{L^{\infty}} .
$$

As

$$
\xi(\alpha, Q, D, \varepsilon, n) \leqq C_{n}\left\|(1-\Delta)^{\frac{1}{2} a(Y)} f\right\|_{E_{\mathscr{C}(Y)}}\left\|(1-\Delta)^{\frac{1}{2} b(Y)} f\right\|_{E}^{n-1},
$$

where the constant $C_{n}$ depends on $\alpha, Q, D, \varepsilon$. Similarly, we have

$$
\eta(\alpha, Q, D, \varepsilon, n) \leqq C_{n}\left\|(1-\Delta)^{\frac{1}{2} a(Y)} f\right\|_{E_{\mathscr{C}(Y)}}\left\|(1-\Delta)^{\frac{1}{2} b(Y)} f\right\|_{E}^{n-1} .
$$

Let the degree of the polynomials $T_{Y},|Y|=L$ be bounded by $\chi_{L}+2$. As before, it now follows, after summation on $\alpha, Q, D, \varepsilon, n$, from (2.47), (2.48), and Theorem 2.6 that

$$
\begin{aligned}
\left\|\widetilde{T}_{Y}(f)\right\|_{E} \leqq & C_{L}\left\|(1-\Delta)^{\frac{1}{2} a(Y)} f\right\|_{E_{\mathscr{P}(Y)}}\left\|(1-\Delta)^{\frac{1}{2} b(Y)} f\right\|_{E} \\
& \times\left(1+\left\|(1-\Delta)^{\frac{1}{2} b(Y)} f\right\|_{E_{\mathscr{Q}(Y)}}\right)^{\chi_{L}}, \quad|Y|=L .
\end{aligned}
$$

This proves (2.41).

Lemma 2.9. Let $f \in E_{\infty}, L \geqq 2$ and let $Y=X_{1}, \ldots, X_{L}$, where $X_{1}, \ldots, X_{L} \in \Pi$. If $\mathscr{L}(Y)=|Y|$ then

$$
\begin{aligned}
& \left\|\widetilde{T}_{Y}(f)\right\|_{E} \\
& \quad \leqq C_{|Y|}\|f\|_{E_{|Y|-1}}\left\|(1-\Delta)^{\frac{1}{2} b^{\prime}(Y)} f\right\|_{E_{1}}\left(1+\left\|(1-\Delta)^{\frac{1}{2} b(Y)} f\right\|_{E}\right) \chi_{|Y|},
\end{aligned}
$$

where

$$
b^{\prime}(Y)=\left[\frac{|Y|-1}{2}\right]+1, \quad b(Y)=\left[\frac{|Y|-1}{2}\right]+2 .
$$

The constants $C_{|Y|}$ and $\chi_{|Y|}$ are independent of $f$.

Proof. As in the proof of Lemma 2.9 it is sufficient to estimate $\xi$ defined by (2.42). According to (2.42) we have after a permutation,

$$
\operatorname{deg} Q_{l} \leqq \operatorname{deg} Q_{1} \leqq|Y|-1, \quad 1 \leqq l \leqq n .
$$

then

$$
\begin{aligned}
\left\|Q_{l} D_{l} f_{\varepsilon_{l}}\right\|_{L^{\infty}} & \leqq C\left\|(1-\Delta) Q_{l} D_{l} f_{\varepsilon_{l}}\right\|_{L^{2}} \\
& \leqq C^{\prime}\left\|(1-\Delta) Q_{l} f\right\|_{E} \\
& \leqq C^{\prime \prime}\left\|(1-\Delta)^{\frac{1}{2} b(Y)} f\right\|_{E}, \quad 2 \leqq l \leqq n
\end{aligned}
$$

and as

$$
\begin{aligned}
\left\|x^{\alpha}\left(Q_{1} D_{1} f_{\varepsilon_{1}}\right)\right\|_{L^{2}} & \leqq\left\|\left(Q_{1} D_{1} f\right)\right\|_{E} \leqq C\left\|D_{1} Q_{1} f\right\|_{E_{|\alpha|}} \\
& \leqq C^{\prime}\left\|Q_{1} f\right\|_{E_{|\alpha|}} \leqq C^{\prime \prime}\left\|(1-\Delta)^{\frac{1}{2} a(Y)} f\right\|_{E_{\mathscr{L}_{(Y)}}}
\end{aligned}
$$

we obtain for $|Y| \geqq 2$ and $\mathscr{L}(Y) \leqq|Y|-1$ :

$$
\begin{aligned}
\xi(\alpha, Q, D, \varepsilon, n) & =\left\|x^{\alpha} \prod_{l=1}^{n}\left(Q_{l} D_{l} f_{\varepsilon_{l}}\right)\right\|_{L^{2}} \\
& \leqq\left\|x^{\beta} Q_{1} D_{1} f_{\varepsilon_{1}}\right\|_{L^{2}}\left\|x^{\gamma} Q_{2} D_{2} f_{\varepsilon_{2}}\right\|_{L^{\infty}} \prod_{l=3}^{n}\left\|Q_{l} D_{l} f_{\varepsilon_{l}}\right\|_{L^{\infty}},
\end{aligned}
$$


where $\beta+\gamma=\alpha$ and where the product over $l$ is absent if $n=2$. We choose $\beta$ and $\gamma$ such that

$$
|\beta| \leqq|Y|-1, \quad|\gamma| \leqq 1
$$

which is possible as $|\alpha| \leqq \mathscr{L}(Y)=|Y|$. It follows from (2.4), (2.50), and (2.52) that

$$
\left\|x^{\beta} Q_{1} D_{1} f_{\varepsilon_{1}}\right\|_{L^{2}} \leqq\left\|x^{\beta} Q_{1} D_{1} f\right\|_{E} \leqq C_{|Y|-1}\left\|D_{1} f\right\|_{E_{|Y|-1}} .
$$

This gives together with (2.3) (and with a new constant $C_{|Y|-1}$ ):

$$
\left\|x^{\beta} Q_{1} D_{1} f_{\varepsilon_{1}}\right\|_{L^{2}} \leqq C_{|Y|-1}\|f\|_{E_{|Y|-1}} .
$$

Similarly, we obtain, using moreover that $\|g\|_{L^{\infty}} \leqq C\|(1-\Delta) g\|_{L^{2}}$, that $\operatorname{deg} Q^{\prime} \leqq 1$, where $Q^{\prime}=\left[x^{\gamma}, 1-\Delta\right]$ and that $\operatorname{deg} Q_{2} \leqq\left[\frac{|Y|-1}{2}\right]$,

$$
\begin{aligned}
\left\|x^{\gamma} Q_{2} D_{2} f_{\varepsilon_{2}}\right\|_{L^{\infty}} & \leqq C\left\|(1-\Delta) x^{\gamma} Q_{2} D_{2} f_{\varepsilon_{2}}\right\|_{L^{2}} \\
& \leqq C\left(\left\|x^{\gamma} Q_{2}(1-\Delta) D_{2} f\right\|_{E}+\left\|Q^{\prime} Q_{2} D_{2} f\right\|_{E}\right) \\
& \leqq C^{\prime}\left\|(1-\Delta)^{\frac{1}{2} b^{\prime}(Y)} f\right\|_{E_{1}} .
\end{aligned}
$$

As $\operatorname{deg} Q_{l} \leqq\left[\frac{|Y|-1}{2}\right]$, for $l \geqq 3$ we have

$$
\left\|Q_{l} D_{l} f_{\varepsilon_{l}}\right\|_{L^{\infty}} \leqq C\left\|(1-\Delta) Q_{l} D_{l} f_{\varepsilon_{l}}\right\|_{L^{2}} \leqq C^{\prime}\left\|(1-\Delta)^{\frac{1}{2} b(Y)} f\right\|_{E}, \quad l \geqq 3 .
$$

Inequalities (2.51), (2.53), (2.54), and (2.55), give for $n \geqq 2$ :

$$
\xi(\alpha, Q, D, \varepsilon, n) \leqq C_{n}\|f\|_{E_{|Y|-1}}\left\|(1-\Delta)^{\frac{1}{2} b^{\prime}(Y)} f\right\|_{E_{1}} \prod_{l=3}^{n}\left\|(1-\Delta)^{\frac{1}{2} b(Y)} f\right\|_{E},
$$

where $C_{n}$ depends on $\alpha, Q, D, \varepsilon$ and where the product over $l$ is absent if $n=2$. As indicated in the proof of Lemma 2.8, inequality (2.56) implies that

$$
\eta(\alpha, Q, D, \varepsilon, n) \leqq C_{n}\|f\|_{E_{|Y|-1}}\left\|(1-\Delta)^{\frac{1}{2} b^{\prime}(Y)} f\right\|_{E_{1}} \prod_{l=3}^{n}\left\|(1-\Delta)^{\frac{1}{2} b(Y)} f\right\|_{E} .
$$

Let the degree of the polynomials $\widetilde{T}_{Y}$ be bounded by $\chi_{|Y|}+2$ for given $|Y|$. Inequality (2.49) now follows from (2.57), similarly as in the proof of (2.40).

Remark 2.10. It follows from the proof of Lemma 2.8 and Lemma 2.9:

i) that $\widetilde{T}_{Y}(f)$ in $(2.40)$ is well defined for $f \in F=L_{\text {loc }}^{2}\left(\mathbb{R}^{2}\right) \oplus L_{\text {loc }}^{2}\left(\mathbb{R}^{2}\right)$ (resp. $\left.E\right)$ and if

$$
\left.(1-\Delta) f \in E \quad \text { [resp. if } f \in E_{\mathscr{L}(Y)} \text { and }(1-\Delta) f \in E\right] \text {; }
$$

ii) that $\widetilde{T}_{Y}(f)$ in (2.41) is well defined for $f \in F$ (resp. $E$ ) and if

$$
\begin{gathered}
(1-\Delta)^{\frac{1}{(}(|Y|-1)} f \in E \text { and }(1-\Delta)^{\frac{1}{2} b(Y)} f \in E \\
{\left[\text { resp. if }(1-\Delta)^{\frac{1}{2} a(Y)} f \in E_{\mathscr{L}(Y)} \text { and }(1-\Delta)^{\frac{1}{2} b(Y)} f \in E\right] ;}
\end{gathered}
$$

iii) that $\widetilde{T}_{Y}(f)$ in (2.49) is well defined for $f \in F$ (resp. $E$ ) and if

$$
(1-\Delta)^{\frac{1}{2}(|Y|-1)} f \in E \text { and }(1-\Delta)^{\frac{1}{2} b(Y)} f \in E
$$

[resp. if $f \in E_{|Y|-1}$ and $(1-\Delta)^{\frac{1}{2}(b(Y)-1)} f \in E_{1}$ and $\left.(1-\Delta)^{\frac{1}{2} b(Y)} f \in E\right]$.

Corollary 2.11. Inequality (2.40) (resp. (2.41), resp. (2.49)) is true if condition (2.58) (resp. (2.59), resp. (2.60)) is satisfied. 
Lemma 2.12. Let $K>0, f \in E_{1}$ and $(1-\Delta) f \in E$. If $\|(1-\Delta) f\|_{E} \leqq K$ and if $K$ is sufficiently small, then

$$
\frac{1}{2}\|f\|_{E_{1}} \leqq \wp_{1}(T(f)) \leqq \frac{3}{2}\|f\|_{E_{1}} .
$$

Proof. It follows from (1.11) and (2.39) that

$$
\begin{gathered}
\|f\|_{E_{1}}=\wp_{1}\left(T^{1}(f)\right) \leqq \wp_{1}(T(f))+\wp_{1}(\tilde{T}(f)), \\
\wp_{1}(T(f)) \leqq\|f\|_{E_{1}}+\wp_{1}(\tilde{T}(f)) .
\end{gathered}
$$

Let $Y$ be as in Lemma 2.8, with $L=1$. Then (2.40) gives

$$
\begin{aligned}
\wp_{1}(\tilde{T}(f)) & =\left(\sum_{|Y| \leqq 1}\left\|\widetilde{T}_{Y}(f)\right\|_{E}^{2}\right)^{1 / 2} \\
& \leqq C_{1}\|(1-\Delta) f\|_{E}\left(1+\|(1-\Delta) f\|_{E}\right)^{\chi_{1}}\left(\sum_{|Y|=1}\|f\|_{\mathscr{E}_{(Y)}}^{2}\right)^{1 / 2},
\end{aligned}
$$

recalling that $\widetilde{T}_{1}=0$. Since $\mathscr{L}(Y) \leqq|Y|$, we get

$$
\wp_{1}(\widetilde{T}(f)) \leqq C\|(1-\Delta) f\|_{E}\left(1+\|(1-\Delta) f\|_{E}\right)^{\chi_{1}}\|f\|_{E_{1}} .
$$

Choosing $K$ such that $C K(1+K)^{\chi_{1}}=\frac{1}{2}$, we get

$$
\wp_{1}(\tilde{T}(f)) \leqq \frac{1}{2}\|f\|_{E_{1}},
$$

which, together with (2.62) and (2.63), proves the lemma.

Before stating the next lemma we remark that for $N \geqq 1$, it makes sense to say that $f \in E$ is such that

$$
\left(\sum_{|\alpha| \leqq N}\left(\wp_{1}\left(\partial^{\alpha} T(f)\right)\right)^{2}\right)^{1 / 2}<\infty .
$$

As a matter of fact it follows from the definition of $T_{Y}$, that $T_{P^{\gamma}}=T_{P^{\gamma}}^{1}=\partial^{\gamma}$ for $\gamma=\left(0, \gamma_{1}, \gamma_{2}\right)$ and then by (2.64) and the definition of $\wp_{1}$ that

$$
\left\|(1-\Delta)^{\frac{1}{2}(N+1)} f\right\|_{E}<\infty .
$$

It follows from (2.65) and Remark 2.10 that if $N=1$, then $\widetilde{T}_{Y}(f)$ is well defined in $F=L_{\text {loc }}^{2} \oplus L_{\text {loc }}^{2}$ for $|Y| \leqq 2$ and that if $N \geqq 2$ then $\widetilde{T}_{Y}(f)$ is well defined in $F$ for $|Y| \leqq N+2$. As $T_{Y}^{1}(f)$ is well defined in $F$ for $|Y| \leqq N+1$ it follows that $T_{Y}(f) \in F$ for $|Y| \leqq N+1$. Hence, $\partial^{\alpha} T_{Y}(f)=T_{P^{\alpha} Y}(f)$ is locally square-integrable for $|Y| \leqq 1$ and $|\alpha| \leqq N$.

Lemma 2.13. Let $f \in E$, and let $\varrho_{N+1}(f)=\left(\sum_{|\alpha| \leqq N}\left(\wp_{1}\left(\partial^{\alpha} T(f)\right)\right)^{2}\right)^{1 / 2}<\infty, N \geqq 1$. Then

$$
\left\|(1-\Delta)^{N / 2} f\right\|_{E_{1}} \leqq\left(\varrho_{N+1}(f)+\|f\|_{E_{1}}\right) C_{N},
$$

where $C_{N}$ is a polynomial in $\left\|(1-\Delta)^{\frac{1}{2}(N+1)} f\right\|_{E}$.

Proof. It follows from (2.6) and the definition of norms that

$$
\left\|(1-\Delta)^{N / 2} f\right\|_{E_{1}} \leqq C_{N} \sum_{|\alpha| \leqq N}\left\|\partial^{\alpha} f\right\|_{E_{1}} \leqq C_{N} \sum_{\substack{|\alpha| \leq N \\|Y| \leqq 1}}\left\|T_{Y}^{1} \partial^{\alpha} f\right\|_{E},
$$

where $Y$ is the unit element in $U(\mathfrak{p})$ or an element of $\Pi$. Since $|Y| \leqq 1$, we have for some constants $C(Y, \alpha, \beta)$ :

$$
\left[Y, P^{\alpha}\right]=\sum_{|\beta| \leqq|\alpha|} C(Y, \alpha, \beta) P^{\beta},
$$

where $\beta=\left(\beta_{0}, \beta_{1}, \beta_{2}\right)$ and $P^{\beta}=P_{0}^{\beta_{0}} P_{1}^{\beta_{1}} P_{2}^{\beta_{2}}$. Hence

$$
T_{Y}^{1} \partial^{\alpha}=\partial^{\alpha} T_{Y}^{1}+\sum_{|\beta| \leqq|\alpha|} C(Y, \alpha, \beta) T_{P \beta}^{1},
$$


which shows, together with (2.67), that

$$
\left\|(1-\Delta)^{N / 2} f\right\|_{E_{1}} \leqq C_{N} \sum_{\substack{|\alpha| \leqq N \\|Y| \leqq 1}}\left\|\partial^{\alpha} T_{Y}^{1} f\right\|_{E},
$$

with $C_{N}$ redefined. As $T=T^{1}+\widetilde{T}$, we obtain from definition (2.38) of the norm $\wp_{1}$ :

$$
\left\|(1-\Delta)^{N / 2} f\right\|_{E_{1}} \leqq C_{N}\left(\varrho_{N+1}(f)+\sum_{\substack{|\alpha| \leqq N \\|Y| \leqq 1}}\left\|\partial^{\alpha} \widetilde{T}_{Y}(f)\right\|_{E}\right) .
$$

It follows from the definition $(1.10)$ that $\partial^{\alpha} \widetilde{T}_{Y}(f)=\widetilde{T}_{P^{\alpha} Y}(f)$, so the last inequality gives:

$$
\left\|(1-\Delta)^{N / 2} f\right\|_{E_{1}} \leqq C_{N}\left(\varrho_{N+1}(f)+\sum_{\substack{|Z| \leqq N+1 \\ \mathscr{L}(Z) \leqq 1}}\left\|\widetilde{T}_{Z}(f)\right\|_{E}\right) .
$$

If $|Z|=0$, then $\widetilde{T}_{Z}(f)=0$. If $|Z|=1$, then it follows from Lemma 2.8 , with $L=0$, that

$$
\left\|\widetilde{T}_{Z}(f)\right\|_{E} \leqq C_{1}^{\prime}\|f\|_{E_{1}}\|(1-\Delta) f\|_{E}\left(1+\|(1-\Delta) f\|_{E}\right)^{\chi_{1}} .
$$

For $Z$ in the domain of summation in (2.69) and $|Z| \geqq 2$, we have $\mathscr{L}(Z) \leqq 1 \leqq|Z|-1$. Hence in this case Lemma 2.8 with $L \geqq 2$ give

$$
\begin{aligned}
\left\|\widetilde{T}_{Z}(f)\right\|_{E} \leqq & C_{|Z|}^{\prime}\left\|(1-\Delta)^{\frac{1}{2}(|Z|-2)} f\right\|_{E_{1}}\left\|(1-\Delta)^{\frac{1}{2} b(Z)} f\right\|_{E} \\
& \times\left(1+\left\|(1-\Delta)^{\frac{1}{2} b(Z)} f\right\|_{E}\right)^{\chi_{|Z|}}, \quad|Z| \geqq 2,
\end{aligned}
$$

where $b(Z)=[(|Z|-1) / 2]+2$. As a matter of fact

$$
\left\|(1-\Delta)^{\frac{1}{2}(|Z|-1)} f\right\|_{E} \leqq C\left\|(1-\Delta)^{\frac{1}{2}(|Z|-2)} f\right\|_{E_{1}} .
$$

Using that $b(Z) \leqq N+1$ for $N \geqq 1$ and $|Z| \leqq N+1$, we obtain (2.70) and (2.71). It follows from (2.70) and (2.71) that

$$
\sum_{\substack{|Z| \leqq N+1 \\ \mathscr{L}(Z) \leqq 1}}\left\|\widetilde{T}_{Z}(f)\right\|_{E} \leqq H_{N}\left(\left\|(1-\Delta)^{\frac{1}{2}(N+1)} f\right\|_{E}\right)\left\|(1-\Delta)^{\frac{1}{2}(N-1)} f\right\|_{E_{1}},
$$

where $H_{N}$ is polynomial with $H_{N}(0)=0$. Inequalities (2.69) and (2.72) give, with $H_{N}$ redefined by a multiplicative factor:

$$
\left\|(1-\Delta)^{N / 2} f\right\|_{E_{1}} \leqq C_{N} \varrho_{N+1}(f)+H_{N}\left\|(1-\Delta)^{\frac{1}{2}(N-1)} f\right\|_{E_{1}}, \quad N \geqq 1 .
$$

After iteration of inequality (2.73) we obtain for $N \geqq 1$ :

$$
\begin{aligned}
\left\|(1-\Delta)^{N / 2} f\right\|_{E_{1}} \leqq & F_{N}\left(\left\|(1-\Delta)^{\frac{1}{2}(N+1)} f\right\|_{E}\right) \varrho_{N+1}(f) \\
& +G_{N}\left(\left\|(1-\Delta)^{\frac{1}{2}(N+1)} f\right\|_{E}\right)\|f\|_{E_{1}},
\end{aligned}
$$

where $F_{N}$ and $G_{N}$ are polynomials and $G_{N}$ has a zero of order $N$ at zero. This proves the lemma.

Theorem 2.14. If $f \in E, N \geqq 1$ and $\wp_{N+1}(T(f))<\infty$, then

$$
\|f\|_{E_{N+1}} \leqq\left(\wp_{N+1}(T(f))+\|f\|_{E_{1}}\right) C_{N+1},
$$

where $C_{N+1}$ is a polynomial in $\wp_{N+1}(T(f))$ and $\|f\|_{E_{1}}$.

Proof. It follows from $T=T^{1}+\widetilde{T}$ and (2.39) that

$$
\|f\|_{E_{N+1}}=\wp_{N+1}\left(T^{1}(f)\right) \leqq \wp_{N+1}(T(f))+\wp_{N+1}(\tilde{T}(f)) .
$$


Similarly, as in the proof of Lemma 2.13 we obtain from Lemma 2.8 and Lemma 2.9 that

$$
\wp_{N+1}(\widetilde{T}(f)) \leqq\|f\|_{E_{N}}\left\|(1-\Delta)^{N / 2} f\right\|_{E_{1}} H_{N}\left(\left\|(1-\Delta)^{\frac{1}{2}(N+1)} f\right\|_{E}\right),
$$

where $H_{N}$ is a polynomial. Observing that for $\varrho_{N+1}$ in Lemma 2.13 we have $\varrho_{N+1}(f) \leqq \wp_{N+1}(T(f))$ we get from the last inequality and Lemma 2.13:

$$
\wp_{N+1}(\widetilde{T}(f)) \leqq\|f\|_{E_{N}}\left(\wp_{N+1}(T(f))+\|f\|_{E_{1}}\right) H_{N}\left(\left\|(1-\Delta)^{\frac{1}{2}(N+1)} f\right\|_{E}\right),
$$

where we have redefined the polynomial $H_{N}$. It follows from inequalities (2.75) and (2.76) that

$$
\begin{aligned}
\|f\|_{E_{N+1}} \leqq & \wp_{N+1}(T(f)) \\
& +H_{N}\left(\left\|(1-\Delta)^{\frac{1}{2}(N+1)} f\right\|_{E}\right)\left(\wp_{N+1}(T(f))+\|f\|_{E_{1}}\right)\|f\|_{E_{N}} \\
\leqq & \left(\wp_{N+1}(T(f))+\|f\|_{E_{1}}\right)\left(1+F_{N+1}\left(\wp_{N+1}(T(f))\|f\|_{E_{N}}\right),\right.
\end{aligned}
$$

where $F_{N+1}$ is a polynomial. Iteration of inequality (2.77) now proves the theorem.

We can now prove that the linear operators $T_{Y}^{1}, Y \in U(\mathfrak{p})$ are bounded by the nonlinear operators $T$, on a neighbourhood of zero in $E_{2}$.

Theorem 2.15. Let $f \in E, N \geqq 2, \wp_{N}(T(f))<\infty$. There is $K>0$, independent of $N$ and $f$, such that if $\|(1-\Delta) f\|_{E} \leqq K$, then

$$
\|f\|_{E_{N}} \wp_{N}(T(f)) H_{N}\left(\wp_{N}(T(f))\right),
$$

where $H_{N}$ is a polynomial independent of $f$.

Proof. According to Lemma 2.12, we choose $K>0$ sufficiently small such that $\|f\|_{E_{1}} \leqq 2 \wp_{1}(T(f)) \leqq 2 \wp_{N}(T(f))$. It follows now from Theorem 2.14 that

$$
\|f\|_{E_{N}} \leqq 3 \wp_{N}(T(f)) C_{N},
$$

where $C_{N}$ is a polynomial in $\wp_{N}(T(f))$ and $\|f\|_{E_{1}}$. We can choose $(a, b) \mapsto C_{N}(a, b)$ such that it is monotonically increasing in each variable for $a, b \geqq 0$. Let $H_{N}(a)$ $=3 C_{N}(a, 2 a)$. This proves the theorem.

It follows from Theorem 2.15 that there is a neighbourhood $O$ of zero in $E_{2}$ such that the differentiable vectors in $O$ of the nonlinear analytic group representation $U$ in $E_{2}$, defined by $T$ are the same as those of the linear group representation $S^{1}$ defined by $T^{1}$. To be more specific let $\mathscr{P}$ be the Poincaré group in $1+2$ dimensions. According to Definition 7 of [1], a differentiable vector of $U$ is an element $f \in E_{2}$ such that the map $g \mapsto U_{g}(f)$ is $C^{\infty}$ from a neighbourhood of the identity in $\mathscr{P}$ to $E_{2}$.

Corollary 2.16. There is a neighbourhood $O$ of zero in $E_{2}$ such that $O \cap E_{\infty}$ are the differentiable vectors of $U$ contained in $O$.

Proof. Let $O_{K}=\left\{g \in E_{2} ;\|g\|_{E_{2}}<K\right\}, K>0$ and let $f \in O_{K}$ be a differentiable vector of $U$. Differentiation of $g \rightarrow U_{g}(g)$ at $g=e$, the identify in $\mathscr{P}_{0}$, in the directions $X_{1}, \ldots, X_{L}, X_{i} \in \mathfrak{p}$ gives the result $T_{Y}(f), Y=X_{1} X_{2}, \ldots, X_{L}$. Since $f$ is a differentiable vector, this shows that $T_{Y}(f) \in E_{2}$ for each $Y \in U(\mathfrak{p})$. In particular, $\wp_{N}(T(f))<\infty$ for each $N \geqq 0$. Since $\|(1-\Delta) f\|_{E} \leqq\|f\|_{E_{2}}<K$ it now follows from Theorem 2.15 that $\|f\|_{E_{N}}<\infty$ for $k$ sufficiently small. Hence $f \in E_{\infty} \cap U_{K}$.

Let $f \in E_{\infty} \cap O_{K}$ and let $K$ be such that $U_{g}$ is analytic on $O_{K}$ for each $g$ in a neighbourhood of the identity in $\mathscr{P}_{0}$. It follows from Theorem 2.6 that $T_{Y}: E_{\infty} \rightarrow E_{\infty}$ for each $Y \in U(p)$, which shows that the map $g \rightarrow U_{g}(f)$ is $C^{\infty}$ at $g=e$ and hence in a neighbourhood of $e$. 


\section{The Second Order Term in the Linearization Map}

The second order term of the equation

$$
T_{X} \circ A=D A \cdot T_{X}^{1}, \quad X \in \mathfrak{p},
$$

gives as usual:

$$
T_{X}^{1} A^{2}-A^{2}\left(T_{X}^{1} \otimes I+I \otimes T_{X}^{1}\right)=-T_{X}^{2}, \quad X \in \mathfrak{p} .
$$

We will prove that there is a unique solution $A^{2} \in L\left(\hat{\otimes}^{2} E_{N}, E_{N}\right)$ if $N$ is sufficiently large and that $A^{2}\left(V_{t} f \otimes V_{t} f\right)$, where $V_{t}=\exp \left(t T_{P_{0}}^{1}\right)$ has certain decrease properties in $E$ and $E^{\infty}$ norms.

We shall denote by $\omega_{M}(k)=\left(M^{2}+|k|^{2}\right)^{1 / 2}, M>0, \omega_{m}=\omega$.

Lemma 3.1. If $M_{1}>0, M_{2}>0, \lambda>-M_{1} M_{2}$ and

$$
Q\left(p_{1}, p_{2}\right)=\lambda+\omega_{M_{1}}\left(p_{1}\right) \omega_{M_{2}}\left(p_{2}\right)-p_{1} \cdot p_{2}, \quad p_{1}, p_{2} \in \mathbb{R}^{2},
$$

then

i) $Q\left(p_{1}, p_{2}\right) \geqq \lambda+M_{1} M_{2}>0$,

ii) a) $Q\left(p_{1}, p_{2}\right) \geqq \lambda+\frac{1}{2} M_{2}^{2} \omega_{M_{1}}\left(p_{1}\right) \omega_{M_{2}}\left(p_{2}\right)^{-1}$ if $\lambda \geqq 0$,

b) $Q\left(p_{1}, p_{2}\right) \geqq \frac{M_{1} M_{2}+\lambda}{M_{1} M_{2}} \frac{1}{2} M_{2}^{2} \omega_{M_{1}}\left(p_{1}\right) \omega_{M_{2}}\left(p_{2}\right)^{-1}$ if $-M_{1} M_{2}<\lambda<0$,

iii) $\left|\nabla_{p_{1}}^{n_{1}} \nabla_{p_{2}}^{n_{2}}\left(Q\left(p_{1}, p_{2}\right)\right)^{-1}\right| \leqq C_{n_{1}, n_{2}} \omega_{M_{2}}\left(p_{2}\right)^{2 n_{1}+n_{2}+1} \omega_{M_{1}}\left(p_{1}\right)^{-\left(n_{1}+1\right)}, n_{1}, n_{2} \geqq 0$.

Proof. For statement i) it is sufficient, due to Lorentz invariance to consider the case where $p_{2}=0$. Then

$$
Q\left(p_{1}, 0\right)=\lambda+\omega_{M_{1}}\left(p_{1}\right) M_{2} \geqq \lambda+M_{1} M_{2}>0 .
$$

For statement ii) we observe that

$$
\begin{aligned}
\omega_{M_{1}}\left(p_{1}\right) \omega_{M_{2}}\left(p_{2}\right)-p_{1} \cdot p_{2} & \geqq \omega_{M_{1}}\left(p_{1}\right) \omega_{M_{2}}\left(p_{2}\right)-\left|p_{1}\right|\left|p_{2}\right| \\
& \geqq \omega_{M_{1}}\left(p_{1}\right)\left(\omega_{M_{2}}\left(p_{2}\right)-\left|p_{2}\right|\right) \\
& =\omega_{M_{1}}\left(p_{1}\right) \frac{M_{2}^{2}}{\omega_{M_{2}}\left(p_{2}\right)+\left|p_{2}\right|} \\
& \geqq \frac{1}{2} M_{2}^{2} \frac{\omega_{M_{1}}\left(p_{1}\right)}{\omega_{M_{2}}\left(p_{2}\right)},
\end{aligned}
$$

which proves the statement if $\lambda \geqq 0$. If $-M_{1} M_{2}<\lambda<0$, then, using i) and ii) with $\lambda=0$, we get

$$
\begin{aligned}
Q\left(p_{1}, p_{2}\right)= & \lambda-\frac{\lambda}{M_{1} M_{2}}\left(\omega_{M_{1}}\left(p_{1}\right) \omega_{M_{2}}\left(p_{2}\right)-p_{1} \cdot p_{2}\right) \\
& +\left(1+\frac{\lambda}{M_{1} M_{2}}\right)\left(\omega_{M_{1}}\left(p_{1}\right) \omega_{M_{2}}\left(p_{2}\right)-p_{1} \cdot p_{2}\right) \\
\geqq & \left(1+\frac{\lambda}{M_{1} M_{2}}\right)\left(\omega_{M_{1}}\left(p_{1}\right) \omega_{M_{2}}\left(p_{2}\right)-p_{1} \cdot p_{2}\right) \\
\geqq & \left(1+\frac{\lambda}{M_{1} M_{2}}\right) \frac{1}{2} M_{2}^{2} \omega_{M_{1}}\left(p_{1}\right) \omega_{M_{2}}\left(p_{2}\right)
\end{aligned}
$$


which proves statement ii) for $-M_{1} M_{2}<\lambda<0$. To prove statement iii) we first observe that, for $p_{1}, p_{2} \in \mathbb{R}^{2}, n_{1} \geqq 0, n_{2} \geqq 0$,

$$
\left|\nabla_{p_{1}}^{n_{1}} \nabla_{p_{2}}^{n_{2}} Q\left(p_{1}, p_{2}\right)\right| \leqq C_{n_{1}, n_{2}} \omega_{M_{1}}\left(p_{1}\right)^{1-n_{1}} \omega_{M_{2}}\left(p_{2}\right)^{1-n_{2}}
$$

Leibnitz rule gives:

$$
\begin{aligned}
& \left|\nabla_{p_{1}}^{n_{1}} \nabla_{p_{2}}^{n_{2}} \frac{1}{Q\left(p_{1}, p_{2}\right)}\right| \leqq C_{n_{1}, n_{2}} \sum_{r=0}^{n_{1}+n_{2}} \sum_{\substack{|i|=n_{1} \\
|j|=n_{2}}}\left(Q\left(p_{1}, p_{2}\right)\right)^{-(r+1)}\left|\nabla_{p_{1}}^{i_{1}} \nabla_{p_{2}}^{j_{1}} Q\right| \ldots\left|\nabla_{p_{1}}^{i_{r}} \nabla_{p_{2}}^{j_{r}} Q\right|, \\
& \left|\nabla_{p_{1}}^{n_{1}} \nabla_{p_{2}}^{n_{2}} \frac{1}{Q\left(p_{1}, p_{2}\right)}\right| \leqq C_{n_{1}, n_{2}} \sum_{r=0}^{n_{1}+n_{2}}\left(Q\left(p_{1}, p_{2}\right)\right)^{-(r+1)} \omega_{M_{1}}\left(p_{1}\right)^{r-n_{1}} \omega_{M_{2}}\left(p_{2}\right)^{r-n_{2}} .
\end{aligned}
$$

Statement i) and inequality (3.4) give (with a new $C_{n_{1}, n_{2}}$ ):

$$
\begin{aligned}
\left|\nabla_{p_{1}}^{n_{1}} \nabla_{p_{2}}^{n_{2}} \frac{1}{Q}\right| & \leqq C_{n_{1}, n_{2}} \sum_{r=0}^{n_{1}+n_{2}}\left(\frac{\omega_{M_{2}}\left(p_{2}\right)}{\omega_{M_{1}}\left(p_{1}\right)}\right)^{r+1} \omega_{M_{1}}\left(p_{1}\right)^{r-n_{1}} \omega_{M_{2}}\left(p_{2}\right)^{r-n_{2}} \\
& =C_{n_{1}, n_{2}} \sum_{r=0}^{n_{1}+n_{2}} \omega_{M_{1}}\left(p_{1}\right)^{-\left(n_{1}+1\right)} \omega_{M_{2}}\left(p_{2}\right)^{2 r+1-n_{2}} \\
& \leqq C_{n_{1}, n_{2}}^{\prime} \omega_{M_{2}}\left(p_{2}\right)^{2 n_{1}+n_{2}+1} \omega_{M_{1}}\left(p_{1}\right)^{-\left(n_{1}+1\right)}
\end{aligned}
$$

which proves statement iii) of the lemma.

Lemma 3.2. Let $\varepsilon, \varepsilon_{1}, \varepsilon_{2}= \pm 1, p_{1}, p_{2} \in \mathbb{R}^{2}$. Then

ii)

$$
\begin{gathered}
\left|\left(\varepsilon \omega\left(p_{1}+p_{2}\right)-\varepsilon_{1} \omega\left(p_{1}\right)-\varepsilon_{2} \omega\left(p_{2}\right)\right)^{-1}\right| \leqq C \omega\left(p_{2}\right)^{2} \\
\left|\nabla_{p_{1}}^{n_{1}} \nabla_{p_{2}}^{n_{2}}\left(\varepsilon \omega\left(p_{1}+p_{2}\right)-\varepsilon_{1} \omega\left(p_{1}\right)-\varepsilon_{2} \omega\left(p_{2}\right)\right)^{-1}\right| \\
\leqq C_{n_{1}, n_{2}} \omega\left(p_{2}\right)^{2 n_{1}+n_{2}+2} \omega\left(p_{1}\right)^{-n_{1}}
\end{gathered}
$$

Proof. As the two cases $\varepsilon=+1$ and $\varepsilon=-1$ are similar we only consider the case $\varepsilon=+1$. Let $Q$ be defined by (with $M_{1}=M_{2}=m$ in Lemma 2.1)

$$
Q\left(p_{1}, p_{2}\right)=\omega\left(p_{1}\right) \omega\left(p_{2}\right)-p_{1} \cdot p_{2}+\frac{1}{2} \varepsilon_{1} \varepsilon_{2} m^{2}, \quad \varepsilon_{1}, \varepsilon_{2}= \pm \text {. }
$$

Then, by Lemma 2.1:

$$
Q\left(p_{1}, p_{2}\right) \geqq \frac{1}{2} m^{2} \quad \text { and } \quad Q\left(p_{1}, p_{2}\right) \geqq \frac{1}{4} m^{2} \omega\left(p_{1}\right) \omega\left(p_{2}\right)^{-1}, \quad \varepsilon_{1}, \varepsilon_{2}= \pm 1 .
$$

Let

$$
R_{\varepsilon_{1}, \varepsilon_{2}}\left(p_{1}, p_{2}\right)=\left(\omega\left(p_{1}+p_{2}\right)-\varepsilon_{1} \omega\left(p_{1}\right)-\varepsilon_{2} \omega\left(p_{2}\right)\right)^{-1}
$$

Then

$$
R_{\varepsilon_{1}, \varepsilon_{2}}\left(p_{1}, p_{2}\right)=-\frac{\omega\left(p_{1}+p_{2}\right)+\varepsilon_{1} \omega\left(p_{1}\right)+\varepsilon_{2} \omega\left(p_{2}\right)}{\varepsilon_{1} \varepsilon_{2} m^{2}+2 \omega\left(p_{1}\right) \omega\left(p_{2}\right)-2 \varepsilon_{1} \varepsilon_{2} p_{1} \cdot p_{2}} \varepsilon_{1} \varepsilon_{2}
$$

So,

$$
R_{\varepsilon_{1}, \varepsilon_{2}}\left(p_{1}, p_{2}\right)=-\varepsilon_{1} \varepsilon_{2} \frac{\omega\left(p_{1}+p_{2}\right)+\varepsilon_{1} \omega\left(p_{1}\right)+\varepsilon_{2} \omega\left(p_{2}\right)}{2 Q\left(\varepsilon_{1} p_{1}, \varepsilon_{2} p_{2}\right)} .
$$

Using inequality (3.8), we get

$$
\left|R_{\varepsilon_{1}, \varepsilon_{2}}\left(p_{1}, p_{2}\right)\right| \leqq \frac{2}{m^{2}}\left(\frac{\omega\left(p_{1}+p_{2}\right) \omega\left(p_{2}\right)}{\omega\left(p_{1}\right)}+\omega\left(p_{2}\right)+\frac{\omega\left(p_{2}\right)^{2}}{\omega\left(p_{1}\right)}\right)
$$


Now, since

$$
\omega\left(k_{1}\right) \omega\left(k_{2}\right) \geqq \frac{m}{\sqrt{2}} \omega\left(k_{1}+k_{2}\right), \quad k_{1}, k_{2} \in \mathbb{R}^{2}
$$

we get

$$
\begin{aligned}
\left|R_{\varepsilon_{1}, \varepsilon_{2}}\left(p_{1}, p_{2}\right)\right| & \leqq \frac{2}{m^{2}}\left(\frac{\sqrt{2}}{m} \omega\left(p_{2}\right)^{2}+\omega\left(p_{2}\right)+\frac{\omega\left(p_{2}\right)^{2}}{\omega\left(p_{1}\right)}\right) \\
& \leqq \frac{2}{m^{2}}\left(\frac{\sqrt{2}}{m}+\frac{2}{m}\right) \omega\left(p_{2}\right)^{2} \leqq \frac{1}{m} C \omega\left(p_{2}\right)^{2}
\end{aligned}
$$

which proves statement i) of the lemma. To prove statement ii) we first note that by Leibnitz formula and (3.9):

$$
\begin{aligned}
\left|\nabla_{p_{1}}^{n_{1}} \nabla_{p_{2}}^{n_{2}} R_{\varepsilon_{1}, \varepsilon_{2}}\left(p_{1}, p_{2}\right)\right| \leqq & C_{n_{1}, n_{2}} \sum_{\substack{|i|=n_{1} \\
|j|=n_{2}}}\left|\nabla_{p_{1}}^{i_{1}} \nabla_{p_{2}}^{j_{1}}\left(2 Q\left(\varepsilon_{1} p_{1}, \varepsilon_{2} p_{2}\right)\right)^{-1}\right| \\
& \times\left|\nabla_{p_{1}}^{i_{2}} \nabla_{p_{2}}^{j_{2}}\left(\omega\left(p_{1}+p_{2}\right)-\varepsilon_{1} \omega\left(p_{1}\right)-\varepsilon_{2} \omega\left(p_{2}\right)\right)\right|,
\end{aligned}
$$

where $i=\left(i_{1}, i_{2}\right)$ and $j=\left(j_{1}, j_{2}\right)$. Using inequality

$$
\left|\nabla^{l} \omega(p)\right| \leqq C_{l}(\omega(p))^{1-l}
$$

and using (3.11) with $k_{1}=p_{1}+p_{2}, k_{2}=-p_{2}$ we get

$$
\begin{aligned}
\mid \nabla_{p_{1}}^{i_{2}} \nabla_{p_{2}}^{j_{2}}\left(\omega\left(p_{1}+p_{2}\right) \mid\right. & \leqq C_{i_{2}+j_{2}}\left(\omega\left(p_{1}+p_{2}\right)\right)^{1-i_{2}-j_{2}} \\
& \leqq C_{i_{1}, i_{2}}^{\prime}\left(\frac{\omega\left(p_{2}\right)}{\omega\left(p_{1}\right)}\right)^{i_{2}+j_{2}-1}, \quad i_{2}+j_{2} \geqq 1
\end{aligned}
$$

Hence, for $i_{2}+j_{2} \geqq 0$,

$$
\mid \nabla_{p_{1}}^{i_{2}} \nabla_{p_{2}}^{j_{2}}\left(\omega\left(p_{1}+p_{2}\right) \mid \leqq C_{i_{1}+i_{2}} \omega\left(p_{2}\right)^{1+j_{2}+j_{2}} \omega\left(p_{1}\right)^{1-i_{2}-j_{2}} .\right.
$$

For $l=1,2$ and $i_{2}+j_{2} \geqq 0$, we also have,

$$
\mid \nabla_{p_{1}}^{i_{2}} \nabla_{p_{2}}^{j_{2}}\left(\omega\left(p_{l}\right) \mid \leqq C_{i_{2}+j_{2}} \omega\left(p_{2}\right)^{1+i_{2}+j_{2}} \omega\left(p_{1}\right)^{1-i_{2}-j_{2}}\right.
$$

Statement iii) of Lemma 3.1 and inequalities (3.13), (3.14), and (3.15) give:

$$
\begin{aligned}
& \left|\nabla_{p_{1}}^{n_{1}} \nabla_{p_{2}}^{n_{2}} R_{\varepsilon_{1}, \varepsilon_{2}}\left(p_{1}, p_{2}\right)\right| \\
& \quad \leqq C_{n_{1}, n_{2}} \sum_{\substack{|i|=n_{1} \\
|j|=n_{2}}} \omega\left(p_{2}\right)^{2 i_{1}+j_{1}+1} \omega\left(p_{1}\right)^{-i_{1}-1} \omega\left(p_{2}\right)^{1+i_{2}+j_{2}} \omega\left(p_{1}\right)^{1-i_{2}-j_{2}} \\
& \quad \leqq C_{n_{1}, n_{2}} \omega\left(p_{2}\right)^{2 n_{1}+n_{2}+2} \omega\left(p_{1}\right)^{-n_{1}},
\end{aligned}
$$

which proves statement ii) of the lemma.

Introduce the functions

$$
d_{\varepsilon, \varepsilon_{1}, \varepsilon_{2}}\left(p_{1}, p_{2}\right)=\left(\varepsilon \omega\left(p_{1}+p_{2}\right)-\varepsilon_{1} \omega\left(p_{1}\right)-\varepsilon_{2} \omega\left(p_{2}\right)\right)^{-1},
$$

$\varepsilon, \varepsilon_{1}, \varepsilon_{2}= \pm 1, p_{1}, p_{2} \in \mathbb{R}^{2}$. As, according to Lemma 3.2 , the functions $d_{\varepsilon, \varepsilon_{1}, \varepsilon_{2}}$ are polynomially bounded together with their derivatives, we can define the linear functions $c_{\varepsilon, \varepsilon_{1}, \varepsilon_{2}}: \mathscr{S}\left(\mathbb{R}^{2}\right) \neq C^{\infty}\left(\mathbb{R}^{2} \times \mathbb{R}^{2}\right)$ by

$$
\left(c_{\varepsilon, \varepsilon_{1}, \varepsilon_{2}}(f)\right)(x, p)=\int e^{i k \cdot x} d_{\varepsilon, \varepsilon_{1}, \varepsilon_{2}}(p, k) \widehat{f}(k) d k \text {. }
$$


For $m \in \mathbb{R}$, let $S^{m}\left(\mathbb{R}^{2} \times \mathbb{R}^{2}\right)$ be the Fréchet space of symbols $f$ satisfying

$$
\left|\nabla_{x}^{s} \nabla_{p}^{t} f(x, p)\right| \leqq C_{s, t}(1+|p|)^{m-t}, \quad s, t \geqq 0 .
$$

Theorem 3.3. If $f \in \mathscr{S}\left(\mathbb{R}^{2}\right)$, then $c_{\varepsilon, \varepsilon_{1}, \varepsilon_{2}}(f) \in S^{0}\left(\mathbb{R}^{2} \times \mathbb{R}^{2}\right)$ and

$$
\left|\nabla_{x}^{s} \nabla_{p}^{t}\left(c_{\varepsilon, \varepsilon_{1}, \varepsilon_{2}}(f)\right)(x, p)\right| \leqq C_{t}\left(\sum_{s \leqq l \leqq 2 t+s+8}\left\|\nabla^{l} f\right\|_{L^{\infty}}^{2}\right)^{1 / 2} \omega(p)^{-t},
$$

$s, t \geqq 0, x, p \in \mathbb{R}^{2}, \varepsilon, \varepsilon_{1}, \varepsilon_{2}= \pm 1$.

Proof. For simplicity we omit the indices $\varepsilon, \varepsilon_{1}, \varepsilon_{2}$ of $d$ and $c$. For given $s, t \geqq 0$ set $a=2 t+8$. Then

$$
2 n_{1}+n_{2}+2-a \leqq-3 \text {, for } 0 \leqq n_{1} \leqq t \text { and } 0 \leqq n_{2} \leqq 3 \text {. }
$$

Lemma 3.2 now gives that

$$
\left|\nabla_{p_{1}}^{n_{1}} \nabla_{p_{2}}^{n_{2}}\left(d\left(p_{1}, p_{2}\right) \omega\left(p_{2}\right)^{-a}\right)\right| \leqq C_{t} \omega\left(p_{2}\right)^{-3} \omega\left(p_{1}\right)^{-n_{1}},
$$

$0 \leqq n_{1} \leqq t, 0 \leqq n_{2} \leqq 3$, which shows that (with a new constant $C_{t}$ )

$$
\int_{\mathbb{R}^{2}}\left|\nabla_{p_{1}}^{n_{1}} \nabla_{p_{2}}^{n_{2}}\left(d\left(p_{1}, p_{2}\right) \omega\left(p_{2}\right)^{-a}\right)\right| d p_{2} \leqq C_{t} \omega\left(p_{1}\right)^{-n_{1}},
$$

for $0 \leqq n_{1} \leqq t, 0 \leqq n_{2} \leqq 3, a=2 t+8$. Introduce

$$
G_{a}(x, p)=\int_{\mathbb{R}^{2}} e^{i k \cdot x} d(p, k) \omega(k)^{-a} d k .
$$

Let $q: \mathbb{R}^{2} \rightarrow \mathbb{C}$ be a polynomial of degree $\leqq 3$. Then, according to (3.20) (with a new constant $C_{t}$ )

$$
\begin{aligned}
& \left|q(x) \nabla_{p}^{t} G_{a}(x, p)\right|=\left|\int e^{i k \cdot x} q\left(-i \nabla_{k}\right) d(p, k) \omega(k)^{-a} d k\right| \\
& \leqq \int\left|q\left(-i \nabla_{k}\right) d(p, k) \omega(k)^{-a}\right| d k \leqq C_{t} \omega\left(p_{1}\right)^{-n_{1}} Q,
\end{aligned}
$$

where $Q$ is the absolute value of the largest coefficient of $q$. As (3.22) is true for all $q$, this gives

$$
\int\left|\nabla_{p}^{t} G_{a}(x, p)\right| d x \leqq C_{t} \omega(p)^{-t}, \quad a=2 t+8 .
$$

It follows from the definitions of $c(f)$ and $G_{a}$ that

$$
\nabla_{x}^{s} \nabla_{p}^{t}(c(f))(x, p)=(2 \pi)^{-1}\left(\left(\nabla_{p}^{t} G_{a}(\cdot, p)\right) \star\left(\nabla^{s} \omega(-i \nabla)^{a} f\right)(\cdot)\right)(x),
$$

where $\star$ is the convolution in the argument of the place of the dot. Inequality (3.23) and Young's inequality give

$$
\left|\nabla_{x}^{s} \nabla_{p}^{t}(c(f))(x, p)\right| \leqq C_{t}\left\|\nabla^{s} \omega(-i \nabla)^{a} f\right\|_{L^{\infty}} \omega(p)^{-t},
$$

for $a=2 t+8$. As $a$ is even we obtain (with a new constant $C_{t}$ )

$$
\left|\nabla_{x}^{s} \nabla_{p}^{t}(c(f))(x, p)\right| \leqq C_{t}\left(\sum_{i=s}^{2 t+s+8}\left\|\nabla^{l} f\right\|_{L^{\infty}}^{2}\right)^{1 / 2} \omega(p)^{-t}
$$

which proves the theorem.

Theorem 3.4. Let $f_{1}, f_{2} \in \mathscr{S}\left(\mathbb{R}^{2}\right)$ and let

$$
\hat{\mathrm{g}}_{\varepsilon, \varepsilon_{1}, \varepsilon_{2}}(k)=\int d_{\varepsilon, \varepsilon_{1}, \varepsilon_{2}}(p, k-p) \hat{f}_{1}(p) \hat{f}_{2}(k-p) d p, \quad \varepsilon, \varepsilon_{1}, \varepsilon_{2}= \pm 1
$$


Then

$$
\left\|g_{\varepsilon, \varepsilon_{1}, \varepsilon_{2}}\right\|_{L^{2}} \leqq C\left(\sum_{s=0}^{q}\left\|\nabla^{s} f_{1}\right\|_{L^{\infty}}^{2}\right)^{1 / 2}\left\|f_{2}\right\|_{L^{2}}
$$

and

$$
\left\|g_{\varepsilon, \varepsilon_{1}, \varepsilon_{2}}\right\|_{L^{2}} \leqq C\left(\sum_{s=0}^{q}\left\|\nabla^{s} f_{2}\right\|_{L^{\infty}}^{2}\right)^{1 / 2}\left\|f_{1}\right\|_{L^{2}},
$$

for some $q \geqq 0$ and $C \geqq 0$, which are independent of $f_{1}$ and $f_{2}$.

Remark 3.5. i) Similar estimates are true if $d$ is replaced by $\nabla_{p_{1}}^{n_{1}} \nabla_{p_{2}}^{n_{2}} d$. One only has to change $q$, and $\left\|f_{j}\right\|_{L^{2}}$ can be replaced by $\left\|(1-\nabla)^{-n_{j / 2}} f_{j}\right\|_{L^{2}}$.

ii) If $a \in C^{\infty}\left(\mathbb{R}^{2} \times \mathbb{R}^{2}\right)$ is polynomially bounded together with its derivatives and

then

$$
\hat{g}(k)=\int a(p, k-p) \hat{f}_{1}(p) \hat{f}_{2}(k-p) d p
$$

$$
\|g\|_{L^{2}} \leqq C\left(\sum_{s=0}^{q}\left\|\nabla^{s} f_{i}\right\|_{L^{\infty}}^{2}\right)^{1 / 2}\left\|(1-\nabla)^{l / 2} f_{j}\right\|_{L^{2}}, \quad(i, j)=(1,2) \text { or }(2,1)
$$

and

$$
\|g\|_{L^{\infty}} \leqq C\left(\sum_{s=0}^{q}\left\|\nabla^{s} f_{1}\right\|_{L^{\infty}}^{2}\right)^{1 / 2}\left(\sum_{s=0}^{q}\left\|\nabla^{s} f_{2}\right\|_{L^{\infty}}^{2}\right)^{1 / 2}
$$

for some $q$ and $l$.

Proof of Theorem 2.4. As the proofs of the two inequalities are analogous, we only consider the case of (3.25). We introduce

$$
g(x)=\frac{1}{2 \pi} \int_{\mathbb{R}^{2}}\left(c\left(f_{2}\right)\right)(x, p) e^{i p \cdot x} \hat{f}_{1}(p) d p,
$$

where $c$ is defined by (3.16). According to Theorem 3.3, $c\left(f_{2}\right) \in S^{-1}(\mathbb{R} \times \mathbb{R})$, so it follows from Theorem 18.1.11 of [3] that $\|g\|_{L^{2}} \leqq C^{\prime}\left\|f_{1}\right\|_{L^{2}}$, where the constant $C^{\prime}$ depends only on a finite number (independent of $f_{1}$ ) of seminorms in $S^{-1}\left(\mathbb{R}^{2} \times \mathbb{R}^{2}\right)$. This means that there exist $s_{0} \geqq 0$ and $t_{0} \geqq 0$ such that

$$
C^{\prime} \leqq \sum_{\substack{0 \leqq s \leqq s_{0} \\ 0 \leqq t \leqq t_{0}}} \sup _{x, p} \omega(p)^{t}\left|\nabla_{x}^{s} \nabla_{p}^{t}\left(c\left(f_{2}\right)\right)(x, p)\right|
$$

It now follows from inequality (3.18) that

$$
C^{\prime} \leqq C_{t_{0}}\left(\sum_{\substack{l \geqq 0 \\ 2 t_{0}+s_{0}+8 \geqq l}}\left\|\nabla^{l} f_{2}\right\|_{L^{\infty}}^{2}\right)^{1 / 2},
$$

where $C_{t_{0}}$ is independent of $f_{2}$. Inequality (3.25) follows by choosing $q=2 t_{0}+s_{0}+8$ and by defining $C=C_{t_{0}}$.

We have a similar result for the estimate of the $L^{\infty}$ norm of $g_{\varepsilon, \varepsilon_{1}, \varepsilon_{2}}$.

Theorem 3.6. Let $g_{\varepsilon, \varepsilon_{1}, \varepsilon_{2}}$ be as in Theorem 3.4. Then there are $q^{\prime}$ and $C^{\prime}$ independent of $f_{1}$ and $f_{2}$ such that

$$
\left\|g_{\varepsilon, \varepsilon_{1}, \varepsilon_{2}}\right\|_{L^{\infty}} \leqq C^{\prime}\left(\sum_{s=0}^{q^{\prime}}\left\|\nabla^{s} f_{2}\right\|_{L^{\infty}}^{2}\right)^{1 / 2}\left(\sum_{s=0}^{q^{\prime}}\left\|\nabla^{s} f_{2}\right\|_{L^{\infty}}^{2}\right)^{1 / 2},
$$

where $\varepsilon, \varepsilon_{1}, \varepsilon_{2}= \pm 1$. 
Proof. For simplicity we omit the indices $\varepsilon, \varepsilon_{1}, \varepsilon_{2}$. For $b \geqq 0$ introduce

$$
\left(F_{b}\left(f_{2}\right)\right)(x, y)=\int c\left(f_{2}\right)(x, p) e^{i p \cdot y} \omega(p)^{-b} d y .
$$

Then, according to (3.26),

$$
g(x)=\int\left(F_{b}\left(f_{2}\right)\right)(x, x-y)\left(\omega(-i \nabla)^{b} f_{1}\right)(y) d y .
$$

It follows from Theorem 3.3 that

$$
\mid \nabla_{p}^{t}\left(c\left(f_{2}\right)(x, p) \omega(p)^{-b} \mid \leqq C_{t}\left(\sum_{l=0}^{2 t+8}\left\|\nabla^{l} f_{2}\right\|_{L^{\infty}}^{2}\right)^{1 / 2} \omega(p)^{-t-b},\right.
$$

which shows that $\nabla_{p}^{t} \mathrm{c}\left(f_{2}\right)(x, p) \omega(p)^{-b}$ is $L^{1}$ in $p$, if $b \geqq 3$, and that

$$
\left|y^{\alpha}\right|\left|\left(F_{b}\left(f_{2}\right)\right)(x, y)\right| \leqq C_{|\alpha|}\left(\sum_{l=0}^{2|\alpha|+8}\left\|\nabla^{l} f_{2}\right\|_{L^{\infty}}^{2}\right)^{1 / 2}, \quad b \geqq 3,
$$

where $\alpha=\left(\alpha_{1}, \alpha_{2}\right)$. This gives

$$
\int\left|\left(F_{b}\left(f_{2}\right)\right)(x, y)\right| d y \leqq C\left(\sum_{l=0}^{14}\left\|\nabla^{l} f_{2}\right\|_{L^{\infty}}^{2}\right)^{1 / 2}, \quad b \geqq 3 .
$$

By (3.29) we have

$$
|g(x)| \leqq \int\left|\left(F_{b}\left(f_{2}\right)\right)(x, y)\right| d y\left\|\omega(-i \nabla)^{b} f_{1}\right\|_{L^{\infty}},
$$

which together with (3.31) gives

$$
|g(x)| \leqq C\left(\sum_{l=0}^{14}\left\|\nabla^{l} f_{2}\right\|_{L^{\infty}}^{2}\right)^{1 / 2}\left\|\omega(-i \nabla)^{b} f_{1}\right\|_{L^{\infty}} .
$$

Choosing $b=4$ we get inequality (3.27) with $q^{\prime}=14$.

We now turn to the resolution of Eq. (3.2). Let $f_{i}=\left(f_{i+}, f_{i-}\right) \in \mathscr{S}\left(\mathbb{R}^{2}\right) \oplus \mathscr{S}\left(\mathbb{R}^{2}\right)$, $i=1,2$. Let $D_{\varepsilon, \varepsilon_{1}, \varepsilon_{2}}$ be the map of $\mathscr{S}\left(\mathbb{R}^{4}\right) \rightarrow \mathscr{S}\left(\mathbb{R}^{4}\right)$ defined by

$$
\left(D_{\varepsilon, \varepsilon_{1}, \varepsilon_{2}} g_{1} \otimes g_{2}\right)\left(p_{1}, p_{2}\right)=i d_{\varepsilon, \varepsilon_{1}, \varepsilon_{2}}\left(p_{1}, p_{2}\right) \hat{g}_{1}\left(p_{1}\right) \hat{g}_{2}\left(p_{2}\right),
$$

$g_{1}, g_{2} \in \mathscr{S}\left(\mathbb{R}^{2}\right)$, where $d_{\varepsilon, \varepsilon_{1}, \varepsilon_{2}}$ is given by (3.16). We define $A_{\varepsilon}^{2}, \varepsilon= \pm$, on

$$
\left(\mathscr{S}\left(\mathbb{R}^{2}\right) \oplus \mathscr{S}\left(\mathbb{R}^{2}\right)\right) \hat{\otimes}\left(\mathscr{S}\left(\mathbb{R}^{2}\right) \oplus \mathscr{S}\left(\mathbb{R}^{2}\right)\right)=E_{\infty} \hat{\otimes} E_{\infty}
$$

by

$$
A_{\varepsilon}^{2}\left(f_{1} \otimes f_{2}\right)=\sum_{\varepsilon_{1}, \varepsilon_{2}= \pm 1} T_{P_{0}}^{2} D_{\varepsilon, \varepsilon_{1}, \varepsilon_{2}}\left(f_{1 \varepsilon_{1}} \otimes f_{2 \varepsilon_{2}}\right) .
$$

Let $A^{2}\left(f_{1} \otimes f_{2}\right)=\left(A_{+}\left(f_{1} \otimes f_{2}\right), A_{-}\left(f_{1} \otimes f_{2}\right)\right)$. As $T_{P_{0}}^{2} \in L\left(E_{\infty} \otimes E_{\infty}, E_{\infty}\right)$ it follows that $A^{2}\left(f_{1} \otimes f_{2}\right) \in E_{\infty}$. It follows like in [6] that $A^{2}$ is a solution of Eq. (3.2) in $L\left(E_{\infty} \hat{\otimes} E_{\infty}, E_{\infty}\right)$.

Theorem 3.7. There exist constants $q, q^{\prime}, C, C^{\prime}$ independent of $f_{1}, f_{2} \in E_{\infty}$ such that

$$
\begin{aligned}
& \left\|A^{2}\left(f_{1} \otimes f_{2}\right)\right\|_{E} \leqq C\left(\sum_{s=0}^{q}\left\|\nabla^{s} f_{1}\right\|_{E^{\infty}}^{2}\right)^{1 / 2}\left\|f_{2}\right\|_{E}, \\
& \left\|A^{2}\left(f_{2} \otimes f_{1}\right)\right\|_{E} \leqq C\left(\sum_{s=0}^{q}\left\|\nabla^{s} f_{2}\right\|_{E^{\infty}}^{2}\right)^{1 / 2}\left\|f_{1}\right\|_{E},
\end{aligned}
$$


and

$$
\left\|A^{2}\left(f_{1} \otimes f_{2}\right)\right\|_{E^{\infty}} \leqq C^{\prime}\left(\sum_{s=0}^{q^{\prime}}\left\|\nabla^{s} f_{1}\right\|_{E^{\infty}}^{2}\right)^{1 / 2}\left(\sum_{s=0}^{q^{\prime}}\left\|\nabla^{s} f_{2}\right\|_{E_{\infty}}^{2}\right)^{1 / 2} .
$$

Proof. Let us consider a term

$$
T_{P_{0}}^{2} D_{\varepsilon, \varepsilon_{1}, \varepsilon_{2}}\left(f_{1 \varepsilon_{1}} \otimes f_{2 \varepsilon_{2}}\right)
$$

in the construction (3.33). Due to the definition (1.9a) of $T_{P_{0}}^{2}$ this term is a linear combination of terms $h$ :

$$
\hat{h}(k)=\int d_{\varepsilon, \varepsilon_{1}, \varepsilon_{2}}(p, k-p) \hat{g}_{1}(p) \hat{g}_{2}(k-p),
$$

where $g_{i}$ is one of the elements

$$
\frac{1}{\omega} f_{i \varepsilon_{\imath}}, f_{i \varepsilon_{\imath}}, \frac{\partial_{j}}{\omega} f_{i \varepsilon_{i}}
$$

According to Theorem 3.4 and Theorem 3.6 we then have:

$$
\begin{aligned}
& \|h\|_{L^{2}} \leqq C\left(\sum_{s=0}^{q}\left\|\nabla^{s} g_{1}\right\|_{L^{\infty}}^{2}\right)^{1 / 2}\left\|g_{2}\right\|_{L^{2}}, \\
& \|h\|_{L^{2}} \leqq C\left(\sum_{s=0}^{q}\left\|\nabla^{s} g_{2}\right\|_{L^{\infty}}^{2}\right)^{1 / 2}\left\|g_{1}\right\|_{L^{2}},
\end{aligned}
$$

and

$$
\|h\|_{L^{\infty}} \leqq C^{\prime}\left(\sum_{s=0}^{q^{\prime}}\left\|\nabla^{s} g_{1}\right\|_{L^{\infty}}^{2}\right)^{1 / 2}\left(\sum_{s=0}^{q^{\prime}}\left\|\nabla^{s} g_{2}\right\|_{L^{\infty}}^{2}\right)^{1 / 2}
$$

with $C, C^{\prime}, q, q^{\prime}$ independent of $g_{1}$ and $g_{2}$. Since

$$
\left\|\omega(i \nabla)^{-1} r\right\|_{L^{\infty}} \leqq C\|r\|_{L^{\infty}},
$$

for $r \in \mathscr{S}\left(\mathbb{R}^{2}\right)$, we can replace $g_{i}$ by $f_{i \varepsilon_{i}}$ in the $L^{\infty}$ norms of the preceding estimates of $h$ if we replace $q$ by $q+1$. Using the fact that $\left\|g_{i}\right\|_{L^{2}} \leqq\left\|f_{i \varepsilon_{i}}\right\|_{L^{2}} \leqq\left\|f_{i}\right\|_{E}$ we obtain (with new constants $C$ and $C^{\prime}$ )

$$
\begin{aligned}
& \|h\|_{L^{2}} \leqq C\left(\sum_{s=0}^{q+1}\left\|D^{s} f_{1}\right\|_{L^{\infty}}^{2}\right)^{1 / 2}\left\|f_{2}\right\|_{E}, \\
& \|h\|_{L^{2}} \leqq C\left(\sum_{s=0}^{q+1}\left\|\nabla^{s} f_{2}\right\|_{L^{\infty}}^{2}\right)^{1 / 2}\left\|f_{1}\right\|_{E},
\end{aligned}
$$

and

$$
\|h\|_{L^{\infty}} \leqq C^{\prime}\left(\sum_{s=0}^{q^{\prime}+1}\left\|\nabla^{s} f_{1}\right\|_{L^{\infty}}^{2}\right)^{1 / 2}\left(\sum_{s=0}^{q^{\prime}+1}\left\|\nabla^{s} f_{2}\right\|_{L^{\infty}}^{2}\right)^{1 / 2} .
$$

This proves the theorem after redefining $q$.

Corollary 3.8. There are $\chi$ and $C$ independent of $f_{1}, f_{2} \in E_{\infty}$ such that

$$
\begin{aligned}
& \left\|A^{2}\left(\left(V_{t} \otimes V_{t}\right)\left(f_{1} \otimes f_{2}\right)\right)\right\|_{E} \\
& \quad \leqq C(1+t)^{-1} \min \left(\left\|f_{1}\right\|_{E}\left\|f_{2}\right\|_{E_{\chi}},\left\|f_{1}\right\|_{E_{\chi}}\left\|f_{2}\right\|_{E}\right), \quad t \geqq 0,
\end{aligned}
$$


and

$$
\left\|A^{2}\left(\left(V_{t} \otimes V_{t}\right)\left(f_{1} \otimes f_{2}\right)\right)\right\|_{E^{\infty}} \leqq C(1+t)^{-2}\left\|f_{1}\right\|_{E_{\chi}}\left\|f_{2}\right\|_{E_{\chi}}, \quad t \geqq 0 .
$$

Proof. According to [2] we have

$$
\begin{aligned}
\left\|\nabla^{s} V_{t} f_{i}\right\|_{L^{\infty}} & =\left\|V_{t} \nabla^{s} f_{i}\right\|_{L^{\infty}} \\
& \leqq C(1+t)^{-1}\left\|\nabla_{s} f_{i}\right\|_{E_{2}} \leqq C^{\prime}(1+t)^{-1}\left\|f_{i}\right\|_{E_{s+2}}
\end{aligned}
$$

The corollary now follows from Theorem 3.7.

Theorem 3.9. There exist $C_{N}$ independent of $f_{1}, f_{2} \in E_{\infty}$ and $\chi_{0}$ independent of $f_{1}, f_{2}$, $N \geqq 0$ such that

i) $\left\|A^{2}\left(f_{1} \otimes f_{2}\right)\right\|_{E_{N}} \leqq C_{N} \sum_{n_{1}+n_{2} \leqq N} \min \left(\left\|f_{1}\right\|_{E_{n_{1}}}\left\|f_{2}\right\|_{E_{n_{2}+x_{0}}}\left\|f_{1}\right\|_{E_{n_{1}+x_{0}}}\left\|f_{2}\right\|_{E_{n_{2}}}\right)$,

ii) if, moreover, $f_{1}=f_{2}=f$ then $\left\|A^{2}(f \otimes f)\right\|_{E_{N}} \leqq C_{N}\|f\|_{E_{N}}\|f\|_{E_{[N / 2]+x_{0}+1} \text {, }}$,

iii) if, moreover, $N$ is suffciently large then $\left\|A^{2}(f \otimes f)\right\|_{E_{N}} \leqq C_{N}\|f\|_{E_{N}}^{2}$.

Proof. Let $S_{X}=T_{X}^{1} \otimes I+I \otimes T_{X}^{1}$ for $X \in \mathfrak{p}$ and let $S_{Y}, Y \in \mathfrak{p}$ be the natural extension to the enveloping algebra. As

$$
T_{X}^{1} A^{2}=A^{2} S_{X}-T_{X}^{2}, \quad X \in \mathfrak{p},
$$

one proves by induction that for $Y=X_{1} \ldots X_{n}, X_{i} \in \mathfrak{p}$,

$$
\begin{aligned}
T_{Y}^{1} A^{2}= & A^{2} S_{Y}-T_{X_{1}}^{2} S_{X_{2} \ldots X_{n}} \\
& -T_{X_{1}}^{1} T_{X_{2}}^{2} S_{X_{3} \ldots X_{n}}-\ldots-T_{X_{1} \ldots X_{n-2}}^{1} T_{X_{n-1}}^{2} S_{X_{n}}-T_{X_{1} \ldots X_{n-1}}^{1} T_{X_{n}}^{1} .
\end{aligned}
$$

Hence by the definition of the space $E_{i}$ we have

$$
\begin{aligned}
\left\|T_{Y}^{1} A^{2}\left(f_{1} \otimes f_{2}\right)\right\|_{E} \leqq & \left\|A^{2} S_{Y}\left(f_{1} \otimes f_{2}\right)\right\|_{E}+\left\|T_{X_{1}}^{1} S_{X_{2} \ldots X_{n}}\left(f_{1} \otimes f_{2}\right)\right\|_{E_{0}} \\
& +\left\|T_{X_{2}}^{2} S_{X_{3} \ldots X_{n}}\left(f_{1} \otimes f_{2}\right)\right\|_{E_{1}}+\ldots \\
& \ldots+\left\|T_{X_{n-1}}^{2} S_{X_{n}}\left(f_{1} \otimes f_{2}\right)\right\|_{E_{n-2}} \\
& +T_{X_{n}}^{2}\left(f_{1} \otimes f_{2}\right) \|_{E_{n-1}} .
\end{aligned}
$$

If $Z=X_{i_{1}} \ldots X_{i_{l}}$, then there are numerical constants $C\left(Z_{1}, Z_{2}\right)$ such that

$$
S_{Z}\left(f_{1} \otimes f_{2}\right)=\sum_{|Z|=\left|Z_{1}\right|+\left|Z_{2}\right|} C\left(Z_{1}, Z_{2}\right)\left(\left(T_{Z_{1}}^{1} f_{1}\right) \otimes\left(T_{Z_{2}}^{1} f_{2}\right)\right) .
$$

Let $Y_{i}=X_{i} X_{i+1} \ldots X_{n}, 1 \leqq i \leqq n$. Then, according to (3.39) the term $\left\|T_{X_{i}}^{2} S_{Y_{\imath+1}}\left(f_{1} \otimes f_{2}\right)\right\|_{E_{i-1}}$ in (3.38) can be bounded by a sum of terms

$$
\left|C\left(Z_{1}, Z_{2}\right)\right|\left\|T_{X_{i}}^{2}\left(\left(T_{Z_{1}}^{1} f_{1}\right) \otimes\left(T_{Z_{2}}^{1} f_{2}\right)\right)\right\|_{E_{\imath-1}}, \quad \text { where }\left|Z_{1}\right|+\left|Z_{2}\right|=n-i
$$

It follows from Lemma 2.1 that

$$
\begin{aligned}
& \left\|T_{X_{i}}^{2}\left(\left(T_{Z_{1}}^{1} f_{1}\right) \otimes\left(T_{Z_{2}}^{1} f_{2}\right)\right)\right\|_{E_{i-1}} \\
& \quad \leqq C_{i-1}\left(\sum_{j_{1}+j_{2} \leqq i-1} \min \left(\left\|T_{Z_{1}}^{1} f_{1}\right\|_{E_{j_{1}}}\left\|T_{Z_{2}}^{1} f_{2}\right\|_{E_{j_{2}+3}},\left\|T_{Z_{2}}^{1} f_{2}\right\|_{E_{j_{2}}}\left\|T_{Z_{1}}^{1} f_{1}\right\|_{E_{j_{1}+3}}\right)\right) .
\end{aligned}
$$

Let $q_{i}=\left|Z_{i}\right|, i=1,2$. Then

and

$$
\left\|T_{Z_{1}}^{1} f_{1}\right\|_{E_{j_{1}}}\left\|T_{Z_{2}}^{1} f_{2}\right\|_{E_{j_{2}+3}} \leqq\left\|f_{1}\right\|_{E_{j_{1}+q_{1}}}\left\|f_{2}\right\|_{E_{j_{2}+q_{2}+3}}
$$

$$
\left\|T_{Z_{2}}^{1} f_{2}\right\|_{E_{j_{2}}}\left\|T_{Z_{1}}^{1} f_{1}\right\|_{E_{j_{1}+3}} \leqq\left\|f_{1}\right\|_{E_{j_{1}+q_{1}+3}}\left\|f_{2}\right\|_{E_{j_{2}+q_{2}}} \text {. }
$$


Inserting (3.42) and (3.43) into inequality (3.41) and then summing up the terms in (3.40) over $\mathrm{q}_{1}$ and $q_{2}$ such that $q_{1}+q_{2}=n-i$, we get

$$
\left\|T_{X_{i}}^{2} S_{Y_{i+1}}\left(f_{1} \otimes f_{2}\right)\right\|_{E_{i-1}}
$$

We obtain after a change of summation variables:

$$
\begin{aligned}
& \left\|T_{X_{i}}^{2} S_{Y_{i+1}}\left(f_{1} \otimes f_{2}\right)\right\|_{E_{i-1}} \\
& \quad \leqq C_{n_{1}+n_{2} \leqq n-1} \sum_{n_{1}} \min \left(\left\|f_{1}\right\|_{E_{n_{1}}}\left\|f_{2}\right\|_{E_{n_{2}+3}},\left\|f_{1}\right\|_{E_{n_{1}+3}}\left\|f_{2}\right\|_{E_{n_{2}}}\right),
\end{aligned}
$$

for $1 \leqq i \leqq n$, where $S_{Y_{n+1}}=$ Id. For the remaining term, $\left\|A^{2}\left(S_{Y}\left(f_{1} \otimes f_{2}\right)\right)\right\|_{E}$, on the right-hand side of (3.38), equality (3.39) gives:

$$
\left\|A^{2}\left(S_{Y}\left(f_{1} \otimes f_{2}\right)\right)\right\|_{E} \leqq C_{n} \sum_{\left|Z_{1}\right|+\left|Z_{2}\right|=n}\left\|A^{2}\left(\left(T_{Z_{1}}^{1} f_{1}\right) \otimes\left(T_{Z_{2}}^{1} f_{2}\right)\right)\right\|_{E} .
$$

Using Corollary 3.8 and denoting by $q_{i}=\left|Z_{i}\right|$, we obtain

$$
\left\|A^{2}\left(S_{Y}\left(f_{1} \otimes f_{2}\right)\right)\right\|_{E} \leqq C_{n} \sum_{q_{1}+q_{2}=n} \min \left(\left\|f_{1}\right\|_{E_{q_{1}}}\left\|f_{2}\right\|_{E_{\chi+q_{2}}}\left\|f_{1}\right\|_{E_{\chi+q_{1}}}\left\|f_{2}\right\|_{E_{q_{2}}}\right) \text {. }
$$

Choosing $\chi_{0} \geqq 3$ and $\chi_{0} \geqq \chi$, inequalities (3.38), (3.44), and (3.45) give:

$$
\left\|T_{Y}^{1} A^{2}\left(f_{1} \otimes f_{2}\right)\right\|_{E} \leqq C_{n_{n}} \sum_{n_{1}+n_{2} \leqq n} \min \left(\left\|f_{1}\right\|_{E_{n_{1}}}\left\|f_{2}\right\|_{E_{n_{2}+x_{0}}}\left\|f_{1}\right\|_{E_{n_{1}+\chi_{0}}}\left\|f_{2}\right\|_{E_{n_{2}}}\right) \text {. (3.4. }
$$

By the definition of $E_{N}$ this proves that

$$
\left\|A^{2}\left(f_{1} \otimes f_{2}\right)\right\|_{E_{N}} \leqq C_{N} \sum_{n_{1}+n_{2} \leqq N} \min \left(\left\|f_{1}\right\|_{E_{n_{1}}}\left\|f_{2}\right\|_{E_{n_{2}+x_{0}}}\left\|f_{1}\right\|_{E_{n_{1}+x_{0}}}\left\|f_{2}\right\|_{E_{n_{2}}}\right),
$$

which proves the first inequality of the theorem. If $n_{1} \geqq \frac{N}{2}$ then $n_{2} \leqq \frac{N}{2} \leqq\left[\frac{N}{2}\right]+1$ and if $n_{2} \geqq \frac{N}{2}$ then $n_{1} \leqq \frac{N}{2} \leqq\left[\frac{N}{2}\right]+1$. This proves that

$$
\min \left(\|f\|_{E_{n_{1}}}\|f\|_{E_{n_{2}+x_{0}}},\|f\|_{E_{n_{1}+x_{0}}}\|f\|_{E_{n_{2}}} \leqq\|f\|_{E_{N}}\|f\|_{E_{\left[\frac{N}{2}\right]+x_{0}+1}} .\right.
$$

Hence by (3.47) (with a new $C_{N}$ )

$$
\left\|A^{2}\left(f_{1} \otimes f_{2}\right)\right\|_{E_{N}} \leqq C_{N}\|f\|_{E_{N}}\|f\|_{E_{\left[\frac{N}{2}\right]+x_{0}+1}},
$$
which proves the second statement of the theorem. Choosing $N \geqq\left[\frac{N}{2}\right]+\chi_{0}+1$,
we get

$$
\left\|A^{2}\left(f_{1} \otimes f_{2}\right)\right\|_{E_{N}} \leqq C_{N}\|f\|_{E_{N}}^{2},
$$

which proves the third statement of the theorem.

\section{The Linearization Operator}

In order to construct a linearization operator of the nonlinear representation $T$ of $\mathfrak{p}$, we consider equality (1.14) in $E$ satisfied for a sufficiently differentiable solution $a$ 
of (1.15)

$$
\frac{d}{d t} T_{Y(t)}\left(a_{1}(t)\right)=T_{P_{0} Y(t)}\left(a_{1}(t)\right), \quad t \geqq 0,
$$

with $Y(t)$ given by (1.13) for $Y \in \Pi^{\prime}$.

Introduce, for $Y \in \Pi^{\prime}$ :

$$
a_{Y}(t)=T_{Y(t)}\left(a_{1}(t)\right) .
$$

Corollary 2.5 and (4.1) then give

$$
\frac{d}{d t} a_{Y}(t)=T_{P_{0}}^{1} a_{Y}(t)+\sum_{Y, 2}^{\prime} T_{P_{0}}^{2}\left(a_{Y_{1}}(t) \otimes a_{Y_{2}}(t)\right)+\bar{T}_{P_{0} Y}\left(a_{1}(t)\right),
$$

where

$$
\bar{T}_{P_{0} Y}\left(a_{1}(t)\right)=\sum_{n \geqq 3} \sum_{Y, n}^{\prime} T_{P_{0}}^{n}\left(a_{Y_{1}}(t) \otimes \ldots \otimes a_{Y_{n}}(t)\right) .
$$

According to the definition of the sum $\sum_{Y, p}^{\prime}$, Eqs. (4.3) and (4.4) define for given $N \geqq 0$, an evolution equation for the variables $\left\{a_{Y}\right\}_{|Y| \leqq N}$, where $Y \in \Pi^{\prime}$ and $a_{Y} \in E$ for $|Y| \leqq N$. As mentioned in the introduction, we have chosen the linearization operator to be a solution of Eq. (1.18). We therefore study the existence of solutions of (4.3) for which there is $\theta \in E_{N}$ such that

$$
\lim _{t \rightarrow \infty} V_{-t} a_{Y}(t)=\theta_{Y} \in E \text { for }|Y| \leqq N,
$$

where $\theta_{Y}=T_{Y}^{1} \theta$. To do this we first subtract from $a$ the terms of order one and two in $\theta$.

Let $\chi$ be as in Corollary 3.8. We define for $N \geqq N_{0}=2 \chi,|Y| \leqq N$ and $\theta \in E_{N}$ :

$$
a_{Y}^{(1)}(t)=V_{t} \theta_{Y}=V_{t} T_{Y}^{1} \theta, \quad a_{Y}^{(2)}(t)=\sum_{Y, 2}^{\prime} A^{2}\left(V_{t} \theta_{Y_{1}} \otimes V_{t} \theta_{Y_{2}}\right), \quad t \in \mathbb{R} .
$$

When needed, we shall write $a_{Y}^{(i)}(\theta, t), i=1,2$, to indicate the dependence of $a$ on $\theta$.

Lemma 4.1. If $N \geqq N_{0}$ and $\theta \in E_{N}$, then

$$
\wp_{N}\left(a^{(2)}(t)\right) \leqq C_{N}(1+t)^{-1}\|\theta\|_{E_{N}}^{2}, \quad t \geqq 0 .
$$

Proof. Let $|Y| \leqq N$ in (4.6). According to definition (2.15) of $Y_{1}$ and $Y_{2}$ we have $\left|Y_{1}\right|+\left|Y_{2}\right|=|Y| \leqq N$ in (4.6). a) Let $\left|Y_{1}\right| \geqq\left|Y_{2}\right|$. Then $\left|Y_{1}\right| \leqq N$ and $\left|Y_{2}\right| \leqq\left[\frac{N}{2}\right]$. It follows now from Corollary 3.8
that

$$
\begin{aligned}
\left\|A^{2}\left(V_{t} \theta_{Y_{1}} \otimes V_{t} \theta_{Y_{2}}\right)\right\|_{E} & \leqq C(1+t)^{-1}\left\|\theta_{Y_{1}}\right\|_{E}\left\|\theta_{Y_{2}}\right\|_{E_{\chi}} \\
& \leqq C_{N}^{\prime}\|\theta\|_{E_{N}}\|\theta\|_{E_{\left[\frac{N}{2}\right]+x}}(1+t)^{-1}, \quad t \geqq 0,
\end{aligned}
$$
where we have used the equality $\theta_{Y}=T_{Y}^{1} \theta$. Since $\left[\frac{N}{2}\right]+\chi \leqq \frac{N}{2}+\frac{N_{0}}{2} \leqq N$, we
obtain

$$
\left\|A^{2}\left(V_{t} \theta_{Y_{1}} \otimes V_{t} \theta_{Y_{2}}\right)\right\|_{E} \leqq C_{N}^{\prime}\|\theta\|_{E_{N}}^{2}(1+t)^{-1}, \quad t \geqq 0 .
$$


b) If $\left|Y_{1}\right| \leqq\left|Y_{2}\right|$, then we deduce similarly that (4.7) is true.

Summation over $Y_{1}, Y_{2}$ now proves the lemma.

For $N \geqq N_{0},|Y| \leqq N$ and $\theta \in E_{N}$, we introduce

$$
b_{Y}(t)=a_{Y}(t)-a_{Y}^{(1)}(t)-a_{Y}^{(2)}(t), \quad t \geqq 0 .
$$

Supposing that the map $t \mapsto a_{Y}(t) \in E$ is $C^{1}$ and using (3.2), Eq. (4.3) gives:

$$
\begin{aligned}
\frac{d}{d t} b_{Y}(t)= & T_{P_{0}}^{1} b_{Y}(t)+\sum_{Y, 2}^{\prime} T_{P_{0}}^{2}\left(a_{Y_{1}}^{(1)}(t) \otimes a_{Y_{2}}^{(2)}(t)+a_{Y_{1}}^{(2)}(t) \otimes a_{Y_{2}}^{(1)}(t)\right. \\
& +a_{Y_{1}}^{(2)}(t) \otimes a_{Y_{2}}^{(2)}(t)+a_{Y_{1}}^{(1)}(t) \otimes b_{Y_{2}}(t)+b_{Y_{1}}(t) \otimes a_{Y_{2}}^{(1)}(t) \\
& \left.+a_{Y_{1}}^{(2)}(t) \otimes b_{Y_{2}}(t)+b_{Y_{1}}(t) \otimes a_{Y_{2}}^{(2)}(t)+b_{Y_{1}}(t) \otimes b_{Y_{2}}(t)\right) \\
& +\bar{T}_{P_{0} Y}\left(b(t)+a^{(1)}(t)+a^{(2)}(t)\right), \quad t \geqq 0 .
\end{aligned}
$$

Let $\mathscr{E}_{N}, N \geqq 0$, be the Hilbert space of elements $f_{Y} \in E$ for $|Y| \leqq N, Y \in \Pi^{\prime}$ and satisfying $\partial^{\alpha} f_{Y}=f_{P^{\alpha} Y}$ for $|\alpha|+|Y| \leqq N$. The norm in $\mathscr{E}_{N}$ is $\wp_{N}$ defined by (2.38). Let $\mathscr{B}_{N}$ be the Banach space of continuous functions from $\left[0, \infty\left[\right.\right.$ to $\mathscr{E}_{N}$ with norm

$$
q_{N}(b)=\sup _{t \geqq 0}\left((1+t) \wp_{N}(b(t))\right), \quad b \in \mathscr{B}_{N} .
$$

We next introduce functions $F, G, H, U$ which will be proven to be polynomial maps of $E_{N} \times \mathscr{B}_{N}$ into $\mathscr{B}_{N}$, for $N$ sufficiently large. For $|Y| \leqq N, Y \in \Pi^{\prime}, \theta \in E_{N}$ and $t \geqq 0$, let

and let

$$
\begin{aligned}
& \left(F_{Y}(\theta, b)\right)(t)=\sum_{\substack{Y, 2 \\
\left|Y_{2}\right| \leqq|Y|-2}}^{\prime} T_{P_{0}}^{2}\left(a_{Y_{1}}^{(1)}(t) \otimes a_{Y_{2}}^{(2)}(t)+a_{Y_{1}}^{(1)}(t) \otimes b_{Y_{2}}(t)\right) \\
& +\sum_{\substack{Y, 2 \\
\left|Y_{2}\right| \leqq|Y|-2}}^{\prime} T_{P_{0}}^{2}\left(a_{Y_{1}}^{(2)}(t) \otimes a_{Y_{2}}^{(1)}(t)+b_{Y_{1}}(t) \otimes a_{Y_{2}}^{(1)}(t)\right) \\
& +\sum_{Y, 2}^{\prime} T_{P_{0}}^{2}\left(\left(a_{Y_{1}}^{(2)}(t)+b_{Y_{1}}(t)\right) \otimes\left(a_{Y_{2}}^{(2)}(t) \otimes b_{Y_{2}}(t)\right),\right. \\
& \begin{aligned}
\left(G_{Y}(\theta, b)\right)(t)= & \sum_{\substack{Y, 2 \\
\left|Y_{1}\right|>|Y|-2}}^{\prime} T_{P_{0}}^{2}\left(a_{Y_{1}}^{(1)}(t) \otimes a_{Y_{2}}^{(2)}(t)\right) \\
& +\sum_{\substack{Y, 2 \\
\left|Y_{2}\right|>|Y|-2}}^{\prime} T_{P_{0}}^{2}\left(a_{Y_{1}}^{(2)}(t) \otimes\left(a_{Y_{2}}^{(1)}(t)\right),\right.
\end{aligned}
\end{aligned}
$$

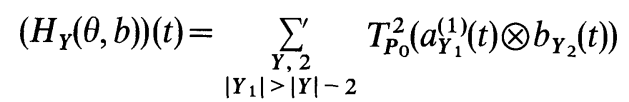

$$
\begin{aligned}
& +\sum_{\substack{Y, 2 \\
\left|Y_{2}\right|>|Y|-2}}^{\prime} T_{P_{0}}^{2}\left(b_{Y_{1}}(t)\right) \otimes\left(a_{Y_{2}}^{(1)}(t)\right),
\end{aligned}
$$

$$
\left(U_{Y}(\theta, b)\right)(t)=\bar{T}_{P_{0} Y}\left(b(t)+a^{(1)}(t)+a^{(2)}(t)\right) .
$$

Equation (4.9) now reads:

$$
\frac{d}{d t} b_{Y}(t)=T_{P_{0}}^{1} b_{Y}(t)+\left(F_{Y}(\theta, b)+G_{Y}(\theta, b)+H_{Y}(\theta, b)+U_{Y}(\theta, b)\right)(t),
$$

where $\theta \in E_{N}$ is given, $b \in \mathscr{B}_{N}$ is unknown, $|Y| \leqq N, t \geqq 0$ and $N$ is sufficiently large. 
Lemma 4.2. There exists $N_{0} \geqq 0$ such that for $N \geqq N_{0}$,

$$
\wp_{N}((F(\theta, b))(t)) \leqq C_{N}(1+t)^{-2}\left(\|\theta\|_{E_{N}}^{2}+\|\theta\|_{E_{N}}^{4}+q_{N}(b)^{2}\right),
$$

and

$$
\wp_{N}((G(\theta, b))(t)) \leqq C_{N}(1+t)^{-2}\|\theta\|_{E_{N}}^{3},
$$

where $\theta \in E_{N}$ and $b \in \mathscr{B}_{N}$.

Proof. Let $\alpha$ and $\beta$ be continuous function from $\left[0, \infty\left[\right.\right.$ into $\mathscr{E}_{N}$. Then $\alpha_{Y}(t)$ and $\beta_{Y}(t)$ are elements of $E$ for $|Y| \leqq N$. Introduce for $\left|Y_{1}\right|,\left|Y_{2}\right| \leqq N$ :

$$
\left(f_{Y_{1}, Y_{2}}(\alpha, \beta)\right)(t)=T_{P_{0}}^{2}\left(\alpha_{Y_{1}}(t) \otimes \beta_{Y_{2}}(t)\right) .
$$

According to inequality (2.7) of Lemma 2.1 we get:

$$
\begin{aligned}
\left.\| f_{Y_{1}, Y_{2}}(\alpha, \beta)\right)(t) \|_{E} & \leqq C \sum_{i, \varepsilon}\left\|\left(B_{i_{1}} \alpha_{Y_{1}}(t)\right)_{\varepsilon_{1}}\left(B_{i_{2}} \beta_{Y_{2}}(t)\right)_{\varepsilon_{2}}\right\|_{L^{2}} \\
& \leqq C^{\prime}(1+t)^{-2} \sum_{B \in \mathscr{B}} \sup _{s \geqq 0}\left((1+s)^{2}\left\|B \alpha_{Y_{1}}(s)\right\|_{E^{\infty}}\left\|\beta_{Y_{2}}(s)\right\|_{E}\right),
\end{aligned}
$$

where $\left\|B \alpha_{Y_{1}}(t)\right\|_{E^{\infty}}\left\|\beta_{Y_{2}}(t)\right\|_{E}$ can be replaced by $\left\|\alpha_{Y_{1}}(t)\right\|_{E}\left\|B \beta_{Y_{2}}(t)\right\|_{E^{\infty}}$. If $\alpha_{Y}(t)=a_{Y}^{(1)}(t)$ $=V_{t} \theta_{Y}, \theta \in E_{N}$, then using $\left\|V_{t} g\right\|_{L^{\infty}} \leqq C(1+|t|)^{-1}\|g\|_{E_{2}}$, we have

$$
\left\|B a_{Y}^{(1)}(t)\right\|_{E^{\infty}}=\left\|V_{t} B \theta_{Y}\right\|_{E^{\infty}} \leqq C(1+t)^{-1}\left\|B \theta_{Y}\right\|_{E_{2}}, \quad t \geqq 0 .
$$

Hence, it follows from (2.3) that (with a new $C$ ):

$$
\begin{aligned}
\left\|B a_{Y}^{(1)}(t)\right\|_{E^{\infty}} & \leqq C(1+t)^{-1}\left\|\theta_{Y}\right\|_{E_{2}} \\
& \leqq(1+t)^{-1}\|\theta\|_{E_{2+|Y|}}, \quad B \in \mathscr{B} .
\end{aligned}
$$

According to (4.19) and (4.20):

$$
\left\|\left(f_{Y_{1}, Y_{2}}\left(a^{(1)}, \beta\right)\right)(t)\right\|_{E} \leqq C(1+t)^{-2}\|\theta\|_{E_{2+\left|Y_{1}\right|}} q_{\left|Y_{2}\right|}(\beta),
$$

where $t \geqq 0$ and $\beta \in \mathscr{B}_{\left|Y_{2}\right|}$. Similarly, we have

$$
\left\|\left(f_{Y_{1}, Y_{2}}\left(\alpha, a^{(1)}\right)\right)(t)\right\|_{E} \leqq C(1+t)^{-2}\|\theta\|_{E_{2+\left|Y_{2}\right|}} q_{\left|Y_{1}\right|}(\alpha),
$$

for $t \geqq 0$ and $\alpha \in \mathscr{B}_{\left|Y_{1}\right|}$. Since $\alpha(t) \in \mathscr{E}_{N}$, we have by definition $\Delta \alpha_{Y}(t)=\alpha_{P_{1}^{2} Y}(t)+\alpha_{P_{2}^{2} Y}(t)$. This gives

$$
\begin{aligned}
\left\|B \alpha_{Y_{1}}(t)\right\|_{E^{\infty}} & \leqq C\left\|(1-\Delta) B \alpha_{Y_{1}}(t)\right\|_{E} \leqq C^{\prime}\left\|(1-\Delta) \alpha_{Y_{1}}(t)\right\|_{E} \\
& \leqq C^{\prime}\left(\left\|\alpha_{Y_{1}}(t)\right\|_{E}+\left\|\alpha_{P_{1}^{2} Y_{1}}(t)\right\|_{E}+\left\|\alpha_{P_{2}^{2} Y_{1}}(t)\right\|_{E}\right) .
\end{aligned}
$$

It follows from the last inequality and inequality (4.19) that

$$
\left\|\left(f_{Y_{1}, Y_{2}}(\alpha, \beta)\right)(t)\right\|_{E} \leqq C(1+t)^{-2} q_{\left|Y_{1}\right|+2}(\alpha) q_{\left|Y_{2}\right|}(\beta),
$$

for $t \geqq 0, \alpha \in \mathscr{B}_{\left|Y_{1}\right|+2}$ and $\beta \in \mathscr{B}_{\left|Y_{2}\right|}$. Similarly,

$$
\left\|\left(f_{Y_{1}, Y_{2}}(\alpha, \beta)\right)(t)\right\|_{E} \leqq C(1+t)^{-2} q_{\left|Y_{1}\right|}(\alpha) q_{\left|Y_{2}\right|+2}(\beta),
$$

for $t \geqq 0, \alpha \in \mathscr{B}_{\left|\mathrm{Y}_{1}\right|}$, and $\beta \in \mathscr{B}_{\left|\mathrm{Y}_{2}\right|+2}$.

We first consider the case of $F$. Let $\left|Y_{1}\right| \leqq|Y|-2$ as in the first sum on the righthand side of (4.11). If

$$
\alpha_{Y}(t)=a_{Y}^{(1)}(t), \quad \beta_{Y}(t)=a_{Y}^{(2)}(t)+b_{Y}(t),
$$


then (4.21) gives, as $2+\left|Y_{1}\right| \leqq|Y| \leqq N$ and $\left|Y_{2}\right| \leqq N$ :

$$
\begin{aligned}
& \sum_{\substack{Y, 2 \\
\left|Y_{1}\right| \leqq|Y|-2}}^{\prime}\left\|\left(f_{Y_{1}, Y_{2}}\left(a^{(1)}, a^{(2)}+b\right)\right)(t)\right\|_{E} \\
& \quad \leqq C_{N}(1+t)^{-2}\|\theta\|_{E_{N}} q_{N}\left(a^{(2)}+b\right) \\
& \leqq C_{N}(1+t)^{-2}\|\theta\|_{E_{N}}\left(q_{N}\left(a^{(2)}\right)+q_{N}(b)\right), \quad t \geqq 0 .
\end{aligned}
$$

Similarly, we obtain for the second sum on the right-hand side of (4.11), where $\left|Y_{2}\right| \leqq|Y|-2$ :

$$
\begin{aligned}
& \sum_{\substack{Y, 2 \\
\left|Y_{2}\right| \leqq|Y|-2}}\left\|\left(f_{Y_{1}, Y_{2}}\left(a^{(2)}+b, a^{(1)}\right)\right)(t)\right\|_{E} \\
& \quad \leqq C_{N}(1+t)^{-2}\|\theta\|_{E_{N}}\left(q_{N}\left(a^{(2)}\right)+q_{N}(b)\right), \quad t \geqq 0 .
\end{aligned}
$$

For the third sum in (4.11), we first consider the terms with $\left|Y_{1}\right| \geqq\left|Y_{2}\right|$. Then $\left|Y_{1}\right| \leqq N$ and $\left|Y_{2}\right|+2 \leqq\left[\frac{N}{2}\right]+2 \leqq N$ for $N \geqq N_{0} \geqq 3$. Let $N_{0} \geqq 3$. It now follows
from (4.25) that

$$
\begin{aligned}
& \sum_{\substack{Y, 2 \\
\left|Y_{1}\right| \geqq\left|Y_{2}\right|}}\left\|\left(f_{Y_{1}, Y_{2}}\left(a^{(2)}+b, a^{(2)}+b\right)\right)(t)\right\|_{E} \\
& \quad \leqq C_{N}(1+t)^{-2}\left(q_{N}\left(a^{(2)}+b\right)\right)^{2} \\
& \quad \leqq C_{N}(1+t)^{-2}\left(q_{N}\left(a^{(2)}\right)+q_{N}(b)\right)^{2}, \quad t \geqq 0 .
\end{aligned}
$$

The estimate (4.28) and its analog for $\left|Y_{1}\right|<\left|Y_{2}\right|$, obtained from (4.24), give for $t \geqq 0$ :

$$
\sum_{Y, 2}^{\prime}\left\|\left(f_{Y_{1}, Y_{2}}\left(a^{(2)}+b, a^{(2)}+b\right)\right)(t)\right\|_{E} \leqq C_{N}(1+t)^{-2}\left(q_{N}\left(a^{(2)}\right)+q_{N}(b)\right)^{2} .
$$

It now follows from estimates (4.26), (4.27), and (4.29) that for $N \geqq N_{0}=3$ :

$$
\begin{aligned}
& (1+t)^{2} \wp_{N}((F(\theta, b))(t) \\
& \quad \leqq C_{N}\left(\|\theta\|_{E_{N}}\left(q_{N}\left(a^{(2)}\right)+q_{N}(b)\right)+\left(q_{N}\left(a^{(2)}\right)+q_{N}(b)\right)^{2}\right) \\
& \quad \leqq C_{N}^{\prime}\left(\|\theta\|_{E_{N}} q_{N}\left(a^{(2)}\right)+\|\theta\|_{E_{N}} q_{N}(b)+\left(q_{N}\left(a^{(2)}\right)\right)^{2}+\left(q_{N}(b)\right)^{2}\right) .
\end{aligned}
$$

Inequality (4.30) and Lemma 4.1 give (with a new $C_{N}$ ):

$$
\begin{aligned}
(1+t)^{2} \wp_{N}((F(\theta, b))(t)) & \leqq C_{N}\left(\|\theta\|_{E_{N}}^{3}+\|\theta\|_{E_{N}}^{4}+\|\theta\|_{E_{N}} q_{N}(b)+\left(q_{N}(b)\right)^{2}\right) \\
& \leqq C_{N}^{\prime}\left(\|\theta\|_{E_{N}}^{2}+\|\theta\|_{E_{N}}^{3}+\|\theta\|_{E_{N}}^{4}+\left(q_{N}(b)\right)^{2}\right) .
\end{aligned}
$$

This proves inequality (4.16).

We now turn to the proof of inequality (4.17). First, let $\left|Y_{2}\right|>|Y|-2$ as in the second sum on the right-hand side of (4.12) and let $\alpha=a^{(2)}$ and $\beta=a^{(1)}$. It then follows from inequality (4.19) that

$$
\begin{aligned}
& \sum_{\substack{Y, 2 \\
\left|Y_{2}\right|>|Y|-2}}^{\prime} \|\left(\left(f_{Y_{1}, Y_{2}}\left(a^{(2)}, a^{(1)}\right)\right)(t) \|_{E}\right. \\
& \quad \leqq C_{N}(1+t)^{-2} \sum_{B \in \mathscr{B}} \sup _{s \geqq 0}\left((1+s)^{2}\left\|B a_{Y_{1}}^{(2)}(s)\right\|_{E^{\infty}}\|\theta\|_{E_{\left|Y_{2}\right|}}\right) .
\end{aligned}
$$

According to (2.3) we have

$$
\left\|B a_{Y_{1}}^{(2)}(s)\right\|_{E^{\infty}} \leqq C\left(\sum_{i=1,2}\left\|\partial_{i} a_{Y_{1}}^{(2)}(s)\right\|_{E^{\infty}}+\left\|a_{Y_{1}}^{(2)}(s)\right\|_{E^{\infty}}\right) .
$$


Using equality $\partial_{i} A^{(2)}(f \otimes g)=A^{(2)}\left(\left(\partial_{i} f\right) \otimes g\right)+A^{(2)}\left(f \otimes \partial_{i} g\right)$ it follows from (4.32), the definition (4.6) of $a^{(2)}$ and Corollary 3.8 that for $\left|Y_{1}\right| \leqq 1$ :

$$
\left\|B a_{Y_{1}}^{(2)}(s)\right\|_{E^{\infty}} \leqq C(1+s)^{-2} \sum_{Z, 2}^{\prime} \sum_{i=1,2}\left(\left\|\partial_{i} \theta_{Z_{1}}\right\|_{E_{\chi_{0}}}\left\|\theta_{Z_{2}}\right\|_{E_{\chi_{0}}}+\left\|\theta_{Z_{1}}\right\|_{E_{\chi_{0}}}\left\|\partial_{i} \theta_{Z_{2}}\right\|_{E_{\chi_{0}}}\right),
$$

for some $\chi_{0} \geqq 0$. Hence, for $\left|Y_{1}\right| \leqq 1$ :

$$
\left\|B a_{Y_{1}}^{(2)}(s)\right\|_{E} \leqq C(1+s)^{-2}\|\theta\|_{E_{N_{0}}}^{2},
$$

where $N_{0}$ is redefined such that it also satisfies $N_{0} \geqq \chi_{0}+2$. Since $N_{0} \leqq N,\left|Y_{2}\right| \leqq N$, $\left|Y_{1}\right| \leqq 1$ we obtain from (4.31) and (4.33),

$$
\sum_{\substack{Y, 2 \\\left|Y_{2}\right|>|Y|-2}}^{\prime}\left\|\left(f_{Y_{1}, Y_{2}}\left(a^{(2)}, a^{(1)}\right)\right)(t)\right\|_{E} \leqq C_{N}(1+t)^{-2}\|\theta\|_{E_{N}}^{3}, \quad N \geqq N_{0} .
$$

Similarly, we obtain that

$$
\sum_{\substack{Y, 2 \\\left|Y_{1}\right|>|Y|-2}}^{\prime}\left\|\left(f_{Y_{1}, Y_{2}}\left(a^{(1)}, a^{(2)}\right)\right)(t)\right\|_{E} \leqq C_{N}(1+t)^{-2}\|\theta\|_{E_{N}}^{3}, \quad N \geqq N_{0} .
$$

Inequalities (4.33) and (4.35) prove that (4.17) is true, which proves the lemma.

Lemma 4.3. If $N \geqq 0$, then

$$
\wp_{N}((H(\theta, b))(t)) \leqq C_{N}(1+t)^{-2}\|\theta\|_{E_{N+2}} q_{N}(b),
$$

for $\theta \in E_{N+2}$ and $b \in \mathscr{B}_{N}$.

Proof. According to (4.21)

$$
\left\|\left(f_{Y_{1}, Y_{2}}\left(a^{(1)}, b\right)\right)(t)\right\|_{E} \leqq C(1+t)^{-2}\|\theta\|_{E_{2+\left|Y_{1}\right|}} q_{\left|Y_{2}\right|}(b) .
$$

Hence, since $\left|Y_{1}\right|,\left|Y_{2}\right| \leqq N$, we get

$$
\sum_{\substack{Y, 2 \\\left|Y_{1}\right|>|Y|-2}}^{\prime}\left\|\left(f_{Y_{1}, Y_{2}}\left(a^{(1)}, b\right)\right)(t)\right\|_{E} \leqq C(1+t)^{-2}\|\theta\|_{E_{N+2}} q_{N}(b) .
$$

Similarly, we obtain

$$
\sum_{\substack{Y, 2 \\\left|Y_{1}\right|>|Y|-2}}^{\prime}\left\|\left(f_{Y_{1}, Y_{2}}\left(b, a^{(1)}\right)\right)(t)\right\|_{E} \leqq C_{N}(1+t)^{-2}\|\theta\|_{E_{N+2}} q_{N}(b) .
$$

Inequalities (4.37) and (4.38) prove the lemma.

Lemma 4.4. There exist $N_{0} \geqq 0$ and $\chi_{N} \geqq 0$ such that

$$
\wp_{N}((U(\theta, b))(t)) \leqq C_{N}(1+t)^{-2}\left(\|\theta\|_{E_{N}}^{2}+\|\theta\|_{E_{N}}^{4}+q_{N}(b)^{2}\right)\left(1+\|\theta\|_{E_{N}}+q_{N}(b)\right)^{\chi_{N}},
$$

for $t \geqq 0, N \geqq N_{0}, \theta \in E_{N}$ and $b \in \mathscr{B}_{N}$.

Proof. Let $Y \in \Pi^{\prime},|Y| \leqq N, n \geqq 3$ and let $T_{P_{0}}^{n}\left(a_{Y_{1}}(t) \otimes \ldots \otimes a_{Y_{n}}(t)\right)$ be a term in (4.4). Then there is $v$ such that $\left|Y_{v}\right| \geqq\left|Y_{j}\right|$ for $1 \leqq j \leqq n$. It follows that $\left|Y_{j}\right| \leqq\left[\frac{|Y|}{2}\right] \leqq\left[\frac{N}{2}\right]$, for $j \neq v$. Hence $\left|Y_{j}\right|+2 \leqq N$ for $N \geqq N_{0} \geqq 3$ and $j \neq v$. Inequality (2.7) of Lemma 2.1 gives:

$$
\left\|T_{P_{0}}^{n}\left(a_{Y_{1}}(t) \otimes \ldots \otimes a_{Y_{n}}(t)\right)\right\|_{E} \leqq C_{N} \sum_{i}\left\|B_{i_{v}} a_{Y_{v}}(t)\right\|_{E} \prod_{l \neq v}\left\|B_{i_{l}} a_{Y_{l}}(t)\right\|_{E^{\infty}}
$$


where $i \in\{1,2,3,4\}^{n}$. According to (4.20), (4.23) with $a^{(2)}=\alpha$ and Lemma 4.1 we have:

$$
\left\|B_{i_{l}} a_{Y_{l}}^{(1)}(t)\right\|_{E^{\infty}} \leqq C(1+t)^{-1}\|\theta\|_{E_{\left|Y_{1}\right|+2}} \leqq C(1+t)^{-1}\|\theta\|_{E_{N}},
$$

and

$$
\left\|B_{i_{l}} a_{Y_{l}}^{(2)}(t)\right\|_{E^{\infty}} \leqq C \wp_{\left|Y_{l}\right|+2}\left(a^{(2)}(t)\right) \leqq C \wp_{N}\left(a^{(2)}(t)\right) \leqq C(1+t)^{-1}\|\theta\|_{E_{N}}^{2},
$$

where $N_{0}$ has been chosen sufficiently large. Similarly, using (4.23) we obtain for $l \neq v$,

$$
\left\|B_{i_{l}} b_{Y_{l}}(t)\right\|_{E^{\infty}} \leqq C(1+t)^{-1} q_{N}(b) .
$$

Since $\left\|B_{i_{v}} a_{Y_{v}}(t)\right\|_{E} \leqq C\left\|a_{Y_{v}}(t)\right\|_{E}$, we have for $a=a^{(1)}+a^{(2)}+b$ :

$$
\begin{aligned}
\left\|B_{i_{v}} a_{Y_{v}}(t)\right\|_{E} & \leqq C\left(\|\theta\|_{E_{N}}+(1+t)^{-1}\|\theta\|_{E_{N}}^{2}+(1+t)^{-1} q_{N}(b)\right) \\
& \leqq C\left(\|\theta\|_{E_{N}}+\|\theta\|_{E_{N}}^{2}+q_{N}(b)\right), \quad N \geqq N_{0},
\end{aligned}
$$

where $N_{0}$ has been chosen sufficiently large. Here we have used Lemma 4.1 for the term $a^{(2)}$. Inequalities (4.39) to (4.43) give:

$$
\begin{aligned}
& \left\|T_{P_{0}}^{n}\left(a_{Y_{1}}(t) \otimes \ldots \otimes a_{Y_{n}}(t)\right)\right\|_{E} \\
& \quad \leqq C_{n}(1+t)^{-(n-1)}\left(\|\theta\|_{E_{N}}+\|\theta\|_{E_{N}}^{2}+q_{N}(b)\right)^{n}, \quad t \geqq 0, \quad N \geqq N_{0} .
\end{aligned}
$$

Since $T_{P_{0}}$ is a polynomial, we obtain from (4.4) and (4.44):

$$
\begin{aligned}
& \left\|\bar{T}_{P_{0} Y}\left(a^{(1)}(t)+a^{(2)}(t)+b(t)\right)\right\|_{E} \\
& \quad \leqq \sum_{n \geqq 3} \sum_{Y, n}^{\prime}\left\|T_{P_{0}}^{n}\left(a_{Y_{1}}(t) \otimes \ldots \otimes a_{Y_{n}}(t)\right)\right\|_{E} \\
& \quad \leqq C_{N}(1+t)^{-2}\left(\|\theta\|_{E_{N}}^{2}+\|\theta\|_{E_{N}}^{4}+q_{N}(b)^{2}\right)\left(1+\|\theta\|_{E_{N}}+q_{N}(b)\right)^{\chi_{N}},
\end{aligned}
$$

where $\chi_{N}$ is sufficiently large and $N \geqq N_{0}$. This proves the lemma.

There is a loss of two degrees in the scale of the seminorms in the estimate of $H(\theta, b)$ in Lemma 4.3. This makes it impossible to prove the existence of solutions $b$ of Eq. (4.15) directly by using the method of Picard in a Banach space. However, as we shall see, the properties of $A^{2}$ permit us to overcome this difficulty by a transformation of Eq. (4.15).

For $N$ sufficiently large, let $\theta \in E_{N}$ and let $t \mapsto b(t) \in \mathscr{E}_{N}, t \geqq 0$ be a $C^{1}$ solution of Eq. (4.15). Let $T_{P_{0}}^{2}\left(a_{Y_{1}}^{(1)}(t) \otimes b_{Y_{2}}(t)\right)$ be a term in the first sum of (4.13). Then, it follows from Eqs. (3.2) and (4.15) that:

$$
\begin{aligned}
& \frac{d}{d t} V_{-t} A^{2}\left(V_{t} \theta_{Y_{1}} \otimes b_{Y_{2}}(t)\right) \\
&= V_{-t}\left(-T_{P_{0}}^{1} A^{2}\left(V_{t} \theta_{Y_{1}} \otimes b_{Y_{2}}(t)\right)\right. \\
&+A^{2}\left(\left(T_{P_{0}}^{1} \otimes I+I \otimes T_{P_{0}}^{1}\right)\left(V_{t} \theta_{Y_{1}} \otimes b_{Y_{2}}(t)\right)\right. \\
&+A^{2}\left(V_{t} \theta_{Y_{1}} \otimes\left(F_{Y_{2}}(\theta, b)+G_{Y_{2}}(\theta, b)+H_{Y_{2}}(\theta, b)+U_{Y_{2}}(\theta, b)\right)(t)\right) \\
&= V_{-t} T_{P_{0}}^{2}\left(V_{t} \theta_{Y_{1}} \otimes b_{Y_{2}}(t)\right) \\
&+V_{-t} A^{2}\left(V_{t} \theta_{Y_{1}} \otimes\left(F_{Y_{2}}(\theta, b)+G_{Y_{2}}(\theta, b)+H_{Y_{2}}(\theta, b)+U_{Y_{2}}(\theta, b)\right)(t)\right) .
\end{aligned}
$$


Hence

$$
\begin{aligned}
V_{-t} & T_{P_{0}}^{2}\left(V_{t} \theta_{Y_{1}} \otimes b_{Y_{2}}(t)\right) \\
= & \frac{d}{d t} V_{-t} A^{2}\left(V_{t} \theta_{Y_{1}} \otimes b_{Y_{2}}(t)\right) \\
& -V_{t} A^{2}\left(V_{t} \theta_{Y_{1}} \otimes\left(F_{Y_{2}}(\theta, b)+G_{Y_{2}}(\theta, b)+H_{Y_{2}}(\theta, b)+U_{Y_{2}}(\theta, b)\right)(t)\right) .
\end{aligned}
$$

Similarly, we get for the terms in the second sum of (4.13):

$$
\begin{aligned}
V_{-t} & T_{P_{0}}^{2}\left(b_{Y_{1}}(t) \otimes V_{t} \theta_{Y_{2}}\right) \\
= & \frac{d}{d t} V_{-t} A^{2}\left(b_{Y_{1}}(t) \otimes V_{t} \theta_{Y_{2}}\right) \\
& -V_{-t} A^{2}\left(\left(F_{Y_{1}}(\theta, b)+G_{Y_{2}}(\theta, b)+H_{Y_{1}}(\theta, b)+U_{Y_{2}}(\theta, b)\right)(t) \otimes V_{t} \theta_{Y_{2}}\right) .
\end{aligned}
$$

Introduce, for $t \geqq 0$ :

and

$$
\begin{aligned}
\left(\mathscr{J}_{Y}(\theta, b)\right)(t)= & \sum_{\substack{Y, 2 \\
\left|Y_{1}\right|>|Y|-2}}^{\prime} A^{2}\left(a_{Y_{1}}^{(1)}(t) \otimes b_{Y_{2}}(t)\right) \\
& +\sum_{\substack{Y, 2 \\
\left|Y_{2}\right|>|Y|-2}} A^{2}\left(b_{Y_{1}}(t) \otimes a_{Y_{2}}^{(1)}(t)\right), \quad t \geqq 0,
\end{aligned}
$$

$$
\begin{aligned}
& (h(\theta, b))(t) \\
& =-\sum_{\substack{Y, 2 \\
\left|Y_{1}\right|>|Y|-2}}^{\prime} A^{2}\left(a_{Y_{1}}^{(1)}(t) \otimes\left(F_{Y_{2}}(\theta, b)+G_{Y_{2}}(\theta, b)+H_{Y_{2}}(\theta, b)+U_{Y_{2}}(\theta, b)\right)(t)\right) \\
& \quad-\sum_{\substack{Y, 2 \\
\left|Y_{2}\right|>|Y|-2}}^{\sum^{\prime}} A^{2}\left(\left(F_{Y_{1}}(\theta, b)+G_{Y_{1}}(\theta, b)+H_{Y_{1}}(\theta, b)+U_{Y_{1}}(\theta, b)\right)(t) \otimes a_{Y_{2}}^{(1)}(t)\right)
\end{aligned}
$$

According to (4.45) and (4.46) we then have:

$$
V_{-t}\left(H_{Y}(\theta, b)\right)(t)=V_{-t}(h(\theta, b))(t)+\frac{d}{d t} V_{-t}\left(\mathscr{J}_{Y}(\theta, b)\right)(t), \quad t \geqq 0 .
$$

Substitution of (4.50) into (4.15) and then integration in $t$ give:

$$
\begin{aligned}
b_{Y}(t)= & \left(\mathscr{J}_{Y}(\theta, b)\right)(t) \\
& -\int_{t}^{\infty} V_{t-s}\left(F_{Y}(\theta, b)+G_{Y}(\theta, b)+h_{Y}(\theta, b)+U_{Y}(\theta, b)\right)(s) d s,
\end{aligned}
$$

where $\theta \in E_{N}, b \in \mathscr{B}_{N},|Y| \leqq N, t \geqq 0$, and $N$ is sufficiently large.

Introduce

$$
\begin{aligned}
\left(K_{Y}(\theta, b)\right)(t)= & \left(\mathscr{J}_{Y}(\theta, b)\right)(t) \\
& -\int_{t}^{\infty} V_{t-s}\left(F_{Y}(\theta, b)+G_{Y}(\theta, b)+h_{Y}(\theta, b)+U_{Y}(\theta, b)\right)(s) d s .
\end{aligned}
$$

Equation (4.50) then reads

$$
b=K(\theta, b),
$$

where $b \in \mathscr{B}_{N}, \theta \in E_{N}$, and $N \geqq N_{0}$ for some $N_{0} \geqq 0$. 
Theorem 4.5. There exists $N_{0} \geqq 0$ such that $K$ is a polynomial map from $E_{N} \times \mathscr{B}_{N}$ into $\mathscr{B}_{N}$ and

$$
q_{N}(K(\theta, b)) \leqq C_{N}\left(\|\theta\|_{E_{N}}^{2}+q_{N}(b)^{2}\right)\left(1+\|\theta\|_{E_{N}}+q_{N}(b)\right)^{\chi_{N}},
$$

for $N \geqq N_{0}$ and some constants $C_{N}$ and $\chi_{N}$.

Proof. The proof of this theorem is similar to the proof of the preceding lemmas in many details. For this reason we will omit several details.

Let $\left|Y_{1}\right|>|Y|-2$. Then, in the first sum on the right-hand side of (4.47) and (4.48), we have $\left|Y_{2}\right| \leqq 1$ and $\left|Y_{1}\right| \leqq|Y| \leqq N$. Inequality (3.35) of Theorem 3.7 gives:

$$
\| A^{2}\left(a_{Y_{1}}^{(1)}(t) \otimes b_{Y_{2}}(t)\left\|_{E} \leqq C\right\| a_{Y_{1}}^{(1)}(t)\left\|_{E} \sum_{|\alpha| \leqq q}\right\| \partial^{\alpha} b_{Y_{2}}(t) \|_{E^{\infty}} .\right.
$$

Since $b \in \mathscr{B}_{N}$ we have $\partial^{\alpha} b_{Y_{2}}=b_{P^{\alpha} Y_{2}}$. The Sobolev inequality $\|f\|_{E^{\infty}} \leqq C \sum_{|\alpha| \leqq 2}\left\|\partial^{\alpha} f\right\|_{E}$ and $\left\|a_{Y_{1}}^{(1)}(t)\right\|_{E} \leqq\|\theta\|_{E_{N}}$ now give:

$$
\left\|A^{2}\left(a_{Y_{1}}^{(1)}(t) \otimes b_{Y_{2}}(t)\right)\right\|_{E} \leqq C(1+t)^{-1}\|\theta\|_{E_{N}} q_{N_{0}}(b),
$$

for $t \geqq 0, N \geqq N_{0}$, where we have chosen $N_{0} \geqq 3+q$. Similarly, we obtain

$$
\begin{aligned}
&\left\|A^{2}\left(a_{Y_{1}}^{(1)}(t) \otimes\left(F_{Y_{2}}(\theta, b)+G_{Y_{2}}(\theta, b)+H_{Y_{2}}(\theta, b)+U_{Y_{2}}(\theta, b)\right)(t)\right)\right\|_{E} \\
& \leqq C\|\theta\|_{E_{N}}\left(\wp_{N_{0}}((F(\theta, b))(t))+\wp_{N_{0}}((G(\theta, b))(t))\right. \\
& \quad+\wp_{N_{0}}((H(\theta, b))(t))+\wp_{N_{0}}((U(\theta, b))(t)) .
\end{aligned}
$$

Choosing $N_{0}$ sufficiently large and using the fact that $\|\theta\|_{E_{N_{0}+2} q_{N_{0}}}(b)$ $\leqq \frac{1}{2}\left(\|\theta\|_{E_{N_{0}+2}}^{2}+q_{N_{0}}(b)^{2}\right)$, it follows from Lemmas 4.2, 4.3, and 4.4 that

$$
\begin{aligned}
& \wp_{N_{0}}((F(\theta, b))(t))+\wp_{N_{0}}((G(\theta, b))(t))+\wp_{N_{0}}((H(\theta, b))(t))+\wp_{N_{0}}((U(\theta, b))(t)) \\
& \leqq C_{N_{0}}(1+t)^{-2}\left(\|\theta\|_{E_{N_{0}+2}}^{2}+\|\theta\|_{E_{N_{0}}}^{2}+q_{N_{0}}(b)^{2}\right)\left(1+\|\theta\|_{E_{N_{0}}}+q_{N_{0}}(b)\right)^{\chi_{N_{0}}} .
\end{aligned}
$$

This inequality and inequality (4.55) give after redefinition of $N_{0}$ and $\chi_{N_{0}}$ :

$$
\begin{aligned}
&\left\|A^{2}\left(a_{Y_{1}}^{(1)}(t) \otimes\left(F_{Y_{2}}(\theta, b)+G_{Y_{2}}(\theta, b)+H_{Y_{2}}(\theta, b)+U_{Y_{2}}(\theta, b)\right)(t)\right)\right\|_{E} \\
& \leqq C_{N_{0}}(1+t)^{-2}\|\theta\|_{E N}\left(\|\theta\|_{E_{N_{0}}}^{2}+\|\theta\|_{E_{N_{0}}}^{4}+q_{N_{0}}(b)^{2}\right) \\
& \times\left(1+\|\theta\|_{E_{N_{0}}}+q_{N_{0}}(b)\right)^{\chi_{N_{0}}}, \quad N \geqq N_{0}, \quad t \geqq 0 .
\end{aligned}
$$

Inequality (4.54) and its analog for $\left|Y_{2}\right|>|Y|-2$ give:

$$
q_{N}(\mathscr{J}(\theta, b)) \leqq C_{N}\|\theta\|_{E_{N}} q_{N_{0}}(b), \quad N \geqq N_{0} .
$$

Inequality (4.56) and its analog for $\left|Y_{2}\right|>|Y|-2$ give:

$$
\begin{aligned}
\wp_{N}((h(\theta, b))(t)) \leqq & C_{N}(1+t)^{-2}\|\theta\|_{E_{N}}\left(\|\theta\|_{E_{N_{0}}}^{2}+\|\theta\|_{E_{N_{0}}}^{4}+q_{N_{0}}(b)^{2}\right) \\
& \times\left(1+\|\theta\|_{E_{N_{0}}}+q_{N_{0}}(b)\right)^{\chi_{N_{0}}}, \quad t \geqq 0, \quad N \geqq N_{0} .
\end{aligned}
$$

It follows from the definition (4.52) of $K$ that

$$
\begin{aligned}
& q_{N}(K(\theta, b)) \leqq q_{N}(\mathscr{J}(\theta, b)) \\
& \quad+\frac{1}{2} \sup _{t \geqq 0}\left((1+t)^{2} \wp_{N}((F(\theta, b)+G(\theta, b)+h(\theta, b)+U(\theta, b))(t)) .\right.
\end{aligned}
$$

Lemmas 4.2 and 4.4 and inequalities (4.57), (4.58), (4.59) give:

$$
q_{N}(K(\theta, b)) \leqq C_{N}\left(\|\theta\|_{E_{N}}^{2}+q_{N}(b)^{2}\right)\left(1+\|\theta\|_{E_{N}}+q_{N}(b)\right)^{\chi_{N}}, \quad N \geqq N_{0},
$$

where $\chi_{N}$ is redefined. This proves the theorem. 
Theorem 4.6. There exists $N_{0} \geqq 0$ such that if $N \geqq N_{0}$, then there exists an open neighbourhood $\mathcal{O}_{N}$ of zero in $E_{N}$ such that the equation $b=K(\theta, b)$ has a unique solution $b(\theta) \in \mathscr{B}_{N}$ for each $\theta \in \mathcal{O}_{N}$. The function $b: \mathcal{O}_{N} \rightarrow \mathscr{B}_{N}$ is analytic and has a zero of order three at $\theta=0$. We choose $\mathcal{O}_{i}, i \geqq N_{0}$ such that $\mathcal{O}_{i+1} \subset \mathcal{O}_{i}$.

Proof. According to Theorem 4.5, $K: E_{N} \times \mathscr{B}_{N} \rightarrow \mathscr{B}_{N}, N \geqq N_{0}$ is an analytic map with a zero of order at least two at the point $(\theta, b)=(0,0)$. The map $R$ defined by $R(\theta, b)=b-K(\theta, b)$ is then an analytic map from $E_{N} \times \mathscr{B}_{N}$ to $\mathscr{B}_{N}$ and $D_{2} R(0,0)$ is the identity map on $\mathscr{B}_{N}$. Here $D_{2}$ denotes the derivative with respect to the second argument. Since $E_{N}$ and $\mathscr{B}_{N}$ are Banach spaces it follows from the implicit mapping theorem that there exists a neighbourhood $\mathcal{O}_{N}$ of zero in $E_{N}$ for which the equation $R(\theta, b)=0$ has a unique analytic solution $b: \mathcal{O}_{N} \rightarrow \mathscr{B}_{N} . K(\theta, b)$ considered as a polynomial in $b$ has an expansion

$$
K(\theta, b)=\sum_{n \geqq 1} k_{n}(\theta, b)+k_{0}(\theta),
$$

where $b \mapsto k_{n}(\theta, b)$ is a monomial of degree $n$ from $\mathscr{B}_{N}$ into itself. It follows from the definition of $K$ that the polynomial $\theta \mapsto k_{0}(\theta)$ has a zero of order three at $\theta=0$ and that the polynomial $\theta \mapsto k_{1}(\theta, b)$ has a zero of order at least one. Since the unique solution $\theta \mapsto b(\theta)$ of equation $b(\theta)=K(\theta, b(\theta))$ satisfies $b(\theta)=0$, it now follows by identification of the $n$-homogeneous parts, $n \geqq 1$ of $b(\theta)=K(\theta, b(\theta))$, that $\theta \mapsto b(\theta)$ has a zero of order three at $\theta=0$. Finally, we redefine $\mathcal{O}_{N}$ by $\mathcal{O}_{N} \cap \mathcal{O}_{N-1} \cap \ldots \cap \mathcal{O}_{N_{0}}$. This proves the theorem.

For $N$ sufficiently large, we can now deduce the existence of $C^{1}$ solutions $t \mapsto a(t) \in \mathscr{E}_{N}, t \geqq 0$ with given scattering data at $t=\infty$, of Eq. (4.3). To indicate the $\theta$ dependence of $a(t)$ we shall write $(a(\theta))(t)$. We introduce for $N \geqq 0$ the Banach space $\mathscr{A}_{N}^{+}$of continuous functions $f: \mathbb{R}^{+} \rightarrow \mathscr{E}_{N}$ with norm

$$
\|f\|_{\mathscr{A}_{N}^{+}}=\sup _{t \geqq 0} \wp_{N}(f(t)), \quad N \geqq 0 .
$$

Theorem 4.7. Let $\mathcal{O}_{N}$ and $b$ be as in Theorem 4.6. There exists $N_{0} \geqq 0$ such that if $N \geqq N_{0}$ and $a(\theta)=a^{(1)}(\theta)+a^{(2)}(\theta)+b(\theta), \theta \in \mathcal{O}_{N}$, then

i) $a: \mathcal{O}_{N} \rightarrow \mathscr{A}_{N}^{+}$is an analytic map,

ii) $\lim _{t \rightarrow \infty} \wp_{N}\left((a(\theta))(t)-\left(a^{(1)}(\theta)\right)(t)\right)=0$ for $\theta \in \mathcal{O}_{N}$,

iii) if $\theta \in \mathcal{O}_{N+1}$, then $t \mapsto(a(\theta))(t) \in \mathscr{E}_{N}, t \geqq 0$ is the unique $C^{1}$ solution of Eq. (4.3) satisfying the condition ii). In addition $\dot{a}(\theta) \in \mathscr{A}_{N}^{+}$, where $(\dot{a}(\theta))(t)=\frac{d}{d t}(a(\theta))(t)$.

Proof. We define $N_{0}$ which is larger than that of Lemma 4.1 and that of Theorem 4.6.

It follows trivially from definition (4.6) of $a^{(1)}$ that it is analytic from $E_{N}$ to $\mathscr{A}_{N}^{+}$. According to Lemma 4.1 the map $\theta \mapsto a^{(2)}(\theta)$ is analytic from $E_{N}$ to $\mathscr{A}_{N}^{+}$and according to Theorem 4.5 the map $\theta \mapsto b(\theta)$ is analytic from $\mathcal{O}_{N}$ to $\mathscr{B}_{N}$ and hence $a$ fortiori to $\mathscr{A}_{N}^{+}$. This proves statement i).

Since $a-a^{(1)}=a^{(2)}+b$, Lemma 4.1 and Theorem 4.6 give for $\theta \in \mathcal{O}_{N}$ :

$$
\begin{aligned}
\wp_{N}\left((a(\theta))(t)-\left(a^{(1)}(\theta)\right)(t)\right) & \leqq \wp_{N}\left(\left(a^{(2)}(\theta)\right)(t)\right)+\wp_{N}((b(\theta))(t)) \\
& \leqq(1+t)^{-1}\left(C_{N}\|\theta\|_{E_{N}}^{2}+q_{N}(b(\theta))\right), \quad t \geqq 0 .
\end{aligned}
$$

This proves statement ii), because $b(\theta) \in \mathscr{B}_{N}$ according to Theorem 4.6. 
Let $\theta \in \mathcal{O}_{N+1}$. It follows from (4.6) that $\dot{a}_{Y}^{(i)}(\theta)=a_{P_{0} Y}^{(i)}(\theta)$. Hence it follows as in the proof of statement i) that $\dot{a}^{(i)}(\theta) \in \mathscr{A}_{N}^{+}$. According to Theorem $4.6, b(\theta) \in \mathscr{B}_{N+1}$ which shows that the right-hand side of Eq. (4.15) is a continuous mapping in $t$ from $\left[0, \infty\left[\right.\right.$ to $\mathscr{E}_{N} . b(\theta)$ is a solution of Eq. (4.52) so, by construction, it is also a solution of Eq. (4.15). This proves that $b(\theta) \in \mathscr{A}_{N}^{+}$. The function $t \mapsto(a(\theta))(t) \in \mathscr{E}_{N}$ is then by construction a $C^{1}$ solution of Eq. (4.3). The uniqueness of this solution follows from the uniqueness of $b$ in Theorem 4.6. This proves statement iii).

The next theorem will permit us to solve the Cauchy problem of Eq. (4.3) with $Y=1$ at $t=0$.

Theorem 4.8. There exists $N_{0} \geqq 2$ such that for $N \geqq N_{0}, \theta \mapsto\left(a_{1}(\theta)\right)(0)$ is an invertible analytic map from $\mathcal{O}_{N}$ onto $\mathcal{O}_{N}^{\prime}$, where $\mathcal{O}_{N}$ and $\mathcal{O}_{N}^{\prime}$ are open neighbourhoods of zero in $E_{N}$. Further $\mathcal{O}_{N+1} \subset \mathcal{O}_{N}$.

Proof. We can choose $N_{0}$ in Theorem 4.7 such that $N_{0} \geqq 2$. According to Theorem 4.7 the map $\theta \mapsto(a(\theta))(0)$ is analytic from $\mathcal{O}_{N}$ to $\mathscr{E}_{N}$. We choose $\mathcal{O}_{N_{0}}$ small enough so that $\wp_{N_{0}}((a(\theta))(t)) \leqq K, t \geqq 0$, where $K$ is given by Theorem 2.15. Since $\left\|(1-\Delta)\left(a_{1}(\theta)\right)(0)\right\|_{E} \leqq \wp_{N_{0}}((a(\theta))(0))$ and $\wp_{N}((a(\theta))(0))<\infty$, Theorem 2.15 gives that $\left\|\left(a_{1}(\theta)\right)(0)\right\|_{E_{N}}<\infty$. This proves that $\theta \mapsto\left(a_{1}(\theta)\right)(0)$ is an analytic map from $\mathcal{O}_{N}$ to $E_{N}$. Denote this map by $A: \mathcal{O}_{N} \rightarrow E_{N}$. We have $D A(0)=I, I=$ identity in $E_{N}$. By the inverse mapping theorem there exists an open neighbourhood $\mathcal{O}_{N}^{\prime}$ of zero on which $A^{-1}$ exists and is analytic. We redefine $\mathcal{O}_{N}$ such that $A: \mathcal{O}_{N} \rightarrow \mathcal{O}_{N}^{\prime}$ is an analytic bijection. The last property is true if $\mathcal{O}_{N+1}$ is redefined by $\mathcal{O}_{N+1} \cap \mathcal{O}_{N}$ and $\mathcal{O}_{N+1}^{\prime}$ by $\mathcal{O}_{N+1}^{\prime} \cap \mathcal{O}_{N}^{\prime}$.

Theorem 4.7 and Theorem 4.8 are the main tools we need to solve the Cauchy problem for Eq. (1.15) with data at $t=0$. Let us introduce the Banach space $\mathscr{A}_{N}$ of continuous functions $f: \mathbb{R} \rightarrow \mathscr{E}_{N}$, with norm

$$
\|f\|_{\mathscr{A}_{N}}=\sup _{t \in \mathbb{R}} \wp_{N}(f(t)), \quad N \geqq 0 .
$$

Introduce also the equation

$$
\left(\dot{u}_{Y}(\theta)\right)(t)=T_{P_{0} Y(t)}\left(u_{1}(\theta)\right)(t), \quad t \in \mathbb{R}
$$

with data

$$
\left(u_{Y}(0)\right)(0)=T_{Y}(\theta) \in E, \quad|Y| \leqq N,
$$

where $\left(u_{Y}(\theta)\right)(t)=T_{Y(t)}\left(\left(u_{1}(\theta)\right)(t),\left(\dot{u}_{Y}(\theta)\right)(t)=\frac{d}{d t}\left(u_{Y}(\theta)\right)(t)\right.$, and $Y \in \Pi^{\prime}$. It follows like in (4.3) and (4.4) that Eq. (4.61) is an evolution equation for the unknown $(u(\theta))(t) \in \mathscr{E}_{N}$.

Theorem 4.9. There exist $N_{0} \geqq 0$, open neighbourhoods $\mathcal{O}_{N_{0}}^{0}, \mathcal{O}_{N_{0}}^{+}, \mathcal{O}_{N_{0}}^{-}$of zero in $E_{N_{0}}$, analytic maps $u: \mathcal{O}_{N_{0}}^{0} \rightarrow \mathscr{A}_{N_{0}}, \Omega_{+}: \mathcal{O}_{N_{0}}^{+} \rightarrow \mathcal{O}_{N_{0}}^{0}, \Omega_{-}: \mathcal{O}_{N_{0}}^{-} \rightarrow \mathcal{O}_{N_{0}}^{0}$ and for $N \geqq N_{0}$ open neighbourhoods $\mathcal{O}_{N}^{0}, \mathcal{O}_{N}^{+}, \mathcal{O}_{N}^{-}$of zero in $E_{N}$, with $\mathcal{O}_{N+1}^{0} \subset \mathcal{O}_{N}^{0}, \mathcal{O}_{N+1}^{+} \subset \mathcal{O}_{N}^{+}, \mathcal{O}_{N+1}^{-} \subset \mathcal{O}_{N}^{-}$ such that:

i) $u: \mathcal{O}_{N}^{0} \rightarrow \mathscr{A}_{N}$ is analytic,

ii) The maps $\Omega_{+}: \mathcal{O}_{N}^{+} \rightarrow \mathcal{O}_{N}^{0}, \Omega_{-}: \mathcal{O}_{N}^{-} \rightarrow \mathcal{O}_{N}^{0}$ are analytic bijections and

$$
\lim _{( \pm) t \rightarrow \infty} \wp_{N}\left((u(\theta))(t)-V_{t} T^{1} \Omega_{( \pm)}^{-1}(\theta)\right)=0 \quad \text { for } \quad \theta \in \mathcal{O}_{N}^{0}
$$

iii) $t \mapsto(u(\theta))(t) \in \mathscr{E}_{N}, \theta \in \mathcal{O}_{N+1}^{0}$ is the unique $C^{1}$ solution of Eq. (4.61) with initial conditions (4.62), 
iv) if $\theta \in \mathcal{O}_{N}^{0}$, then $(u(\theta))(t) \in E_{N}$ for $t \in \mathbb{R}$ and the map $t \mapsto V_{-t}\left(u_{1}(\theta)\right)(t)$ defines a continuous function in $t$ from $\mathbb{R}$ into the space of analytic functions from $\mathcal{O}_{N}^{0}$ into $E_{N}$.

Proof. Let us choose $N_{0}^{+}$which is not smaller than $N_{0}$ of Theorem 4.7 or Theorem 4.8. For $N \geqq N_{0}^{+}$we define $\mathcal{O}_{N}^{+}$as the intersection of $\mathcal{O}_{N}$ from Theorem 4.7 and Theorem 4.8. It then follows from Theorem 4.8 that $\theta \mapsto \Omega_{+}(\theta)=\left(a_{1}(\theta)\right)(0)$ is an invertible analytic map from $\mathcal{O}_{N}^{+}$onto $\mathcal{O}_{+, N}^{0}, N \geqq N_{0}^{+}$, where $\mathcal{O}_{+, N}^{0}=\Omega_{+}\left(\mathcal{O}_{N}^{+}\right)$. For $a(\theta)$ given by Theorem 4.7 we denote $a^{+}(\theta)=a(\theta)$.

There is an analog of Theorem 4.7 for Eq. (4.1) with $t \leqq 0$, obtained by considering instead of (4.5) solutions $a^{-}(\theta)$ satisfying

$$
\lim _{t \rightarrow-\infty} V_{-t}\left(a_{Y}^{-}(\theta)\right)(t)=\theta_{Y}=T_{Y} \theta \in E \quad \text { for } \quad|Y| \leqq N .
$$

As above we then obtain $N_{0}^{-}, \mathcal{O}_{N}^{-}$for $N \geqq N_{0}^{-}$and the invertible analytic map $\Omega_{-}$ from $\mathcal{O}_{N}^{-}$onto $\mathcal{O}_{-, N}^{0}$, where $\Omega_{-}(\theta)=\left(a_{1}^{-}(\theta)\right)(0)$.

We define $N_{0}=\max \left(N_{0}^{+}, N_{0}^{-}\right), \mathcal{O}_{N}^{0}=\mathcal{O}_{+, N}^{0} \cap \mathcal{O}_{-, N}^{0}$ and we redefine $\mathcal{O}_{N}^{+}$and $\mathcal{O}_{N}^{-}$by $\mathcal{O}_{N}^{+}=\Omega_{+}^{-1}\left(\mathcal{O}_{N}^{0}\right)$ and $\mathcal{O}_{N}^{-}=\Omega_{-}^{-1}\left(\mathcal{O}_{N}^{0}\right)$, which are open subsets of the old ones. They are neighbourhoods of zero in $E_{N}$ since $\Omega_{( \pm)}(0)=0$.

For $\theta \in \mathcal{O}_{N_{0}}^{0}$ we now define $u(\theta)$ by

$$
(u(\theta))(t)=\left(a^{+}\left(\Omega_{+}^{-1}(\theta)\right)\right)(t) \quad \text { for } \quad t \geqq 0
$$

and

$$
(u(\theta))(t)=\left(a^{-}\left(\Omega_{-}^{-1}(\theta)\right)\right)(t) \quad \text { for } \quad t<0 .
$$

Since $\left(a^{+}(\theta)\right)(0)=T\left(\Omega_{+}(\theta)\right), \theta \in \mathcal{O}_{N}^{+}$and $\left(a^{-}(\theta)\right)(0)=T\left(\Omega_{-}(\theta)\right), \theta \in \mathcal{O}_{N}^{-}$we have $\left(a^{+}\left(\Omega_{+}^{-1}(\theta)\right)\right)(0)=\left(a^{-}\left(\Omega_{+}^{-1}(\theta)\right)\right)(0)=T(\theta)$ in $\mathscr{E}_{N}$ for $\theta \in \mathcal{O}_{N}^{0}$. This proves that $t \mapsto(u(\theta))(t)$ is continuous at $t=0$ and that $(u(\theta))(0)=T(\theta)$, so (4.62) is satisfied.

Statements i) and iii) of the theorem and the equality in the statement (ii) of the theorem now follow from the corresponding statements of Theorem 4.8 and its analog for $t \leqq 0$.

To prove statement iv) of the theorem we remark that we have already fixed $\mathcal{O}_{N}^{0}$, by the definition of $\mathcal{O}_{N}^{\prime}$ in the proof of Theorem 4.8, such that

$$
\left\|(1-\Delta)\left(a_{1}^{+}\left(\Omega_{+}^{-1}(\theta)\right)\right)(t)\right\|_{E} \leqq \wp_{N_{0}}\left(\left(a\left(\Omega_{+}^{-1}(\theta)\right)(t)\right) \leqq K\right.
$$

for $t \geqq 0$ and $\theta \in \Omega_{+}\left(\mathcal{O}_{N}^{+}\right)=\mathcal{O}_{N}^{0}$ and similarly for $t<0$. Here $K$ is given by Theorem 2.15. Hence $\left\|(1-\Delta)\left(u_{1}(\theta)\right)(t)\right\|_{E} \leqq K$ for $t \in \mathbb{R}$. By statement i) it follows that $\wp_{N}((u(\theta))(t))<\infty$ for $\theta \in \mathcal{O}_{N}^{0}$ and $t \in \mathbb{R}$, so by Theorem 2.15 we have $\left\|\left(u_{1}(\theta)\right)(t)\right\|_{E_{N}}$ $<\infty$. The map $\theta \mapsto(u(\theta))(t)$ is analytic according to statement i). The continuity follows from the integral equation corresponding to Eq. (1.15). This proves the theorem.

Theorem 4.10. There exists $N^{\prime} \geqq 0$ such that, if $\mathcal{O}_{N^{\prime}}^{0}, \mathcal{O}_{N^{\prime}}^{+}, \mathcal{O}_{N^{\prime}}^{-}$are given by Theorem 4.9, then

i) it is possible to choose $\mathcal{O}_{N}^{0}=E_{N} \cap \mathcal{O}_{N^{\prime}}^{0}, \mathcal{O}_{N}^{+}=E_{N} \cap \mathcal{O}_{N^{\prime}}^{+}, \mathcal{O}_{N}^{-}=E_{N} \cap \mathcal{O}_{N^{\prime}}^{-}$in Theorem 4.9 for $N \geqq N^{\prime}$,

ii) the invertible analytic maps $\Omega_{+}: \mathcal{O}_{N^{\prime}}^{+} \rightarrow \mathcal{O}_{N^{\prime}}^{0}, \Omega_{-}: \mathcal{O}_{N^{\prime}}^{-} \rightarrow \mathcal{O}_{N^{\prime}}^{0}$ satisfy

$$
D \Omega_{\varepsilon} \cdot T_{X}^{1}=T_{X} \circ \Omega_{\varepsilon} \quad \text { on } \mathcal{O}_{N^{\prime}+1}^{\varepsilon} \text { and for } X \in \mathfrak{p},
$$

where $\varepsilon= \pm$,

iii) if $\mathcal{O}_{\infty}^{0}=\mathcal{O}_{N^{\prime}}^{0} \cap E_{\infty}, \mathcal{O}_{\infty}^{+}=\mathcal{O}_{N^{\prime}}^{+} \cap E_{\infty}, \mathcal{O}_{\infty}^{-}=\mathcal{O}_{N^{\prime}}^{-} \cap E_{\infty}$ then $\Omega_{( \pm)}\left(\mathcal{O}_{\infty}^{( \pm)}\right)=\mathcal{O}_{\infty}^{0}$. 
Proof. Let $N^{\prime}=N_{0}+2$, where $N_{0}$ is given by Theorem 4.9 and redefine $\mathcal{O}_{N_{0}+i}^{\varepsilon}$, $i=0,1,2, \varepsilon=0,+,-$ to be the interior of the closure in $E_{N_{0}+i}$ of $E_{N_{0}+i} \cap \mathcal{O}_{N^{\prime}}^{\varepsilon}$. The open neighbourhood $\mathcal{O}_{N_{0}+i}^{\varepsilon}$ of zero in $E_{N_{0}+i}$ is included in the one defined in Theorem 4.9. Let $\mathcal{O}_{N}^{\varepsilon}$ be given by Theorem 4.9 for $N \geqq N^{\prime}$. Then the conclusion of that theorem is valid for the sequence $\mathcal{O}_{N_{0}}^{\varepsilon}, \mathcal{O}_{N_{0}+1}^{\varepsilon}, \ldots, \mathcal{O}_{N}^{\varepsilon}, \ldots, \varepsilon=0,+,-$.

Let us first consider statement ii) for $\Omega_{+}$. Let $\theta \in \mathcal{O}_{N_{0}+1}$. Then $\Omega_{+}(\theta) \in \mathcal{O}_{N_{0}+1}$ and $T_{X}^{1} \theta \in E_{N_{0}+1}$, so $\theta \mapsto D \Omega_{+}(\theta) \cdot\left(T_{X}^{1} \theta\right)$ and $\theta \mapsto\left(T_{X} \circ \Omega_{+}\right)(\theta)$ are analytic functions from $\mathcal{O}_{N_{0}+1}$ to $E_{N_{0}}$. According to the definition of $\mathcal{O}_{N_{0}+2}$, it is dense in $\mathcal{O}_{N_{0}+1}$. Let $\theta \in \mathcal{O}_{N_{0}+2}$ and let $X \in \mathfrak{p}$. It follows from definition (1.10) and statement ii) and iii) of Theorem 4.7 that $a_{X}^{+}(\theta)$ is the unique solution of the equation

$$
\frac{d}{d t}\left(a_{X}^{+}(\theta)\right)(t)=D T_{P_{0}}\left(\left(a_{1}^{+}(\theta)(t)\right) \cdot\left(a_{X}^{+}(\theta)\right)(t), \quad t \geqq 0,\right.
$$

with $\left.\| a_{X}^{+}(\theta)\right)(t)-V_{t} T_{X}^{1} \theta \|_{E} \rightarrow 0$ as $t \rightarrow \infty$. Introduce $\alpha_{X}^{+}(\theta)=D a_{1}^{+}(\theta) \cdot\left(T_{X}^{1} \theta\right)$. Then differentiation in $\theta$ of the equation

$$
\frac{d}{d t}\left(a_{1}^{+}(\theta)\right)(t)=T_{P_{0}}\left(\left(a_{1}^{+}(\theta)\right)(t)\right)
$$

gives

$$
\frac{d}{d t}\left(\alpha_{X}^{+}(\theta)\right)(t)=D T_{P_{0}}\left(\left(a_{1}^{+}(\theta)\right)(t)\right) \cdot\left(\alpha_{X}^{+}(\theta)\right)(t) .
$$

Since $\lim _{t \rightarrow \infty} V_{-t}\left(a_{1}^{+}(\theta)\right)(t)=\theta$, it follows that

$$
\left\|\left(\alpha_{X}^{+}(\theta)\right)(t)-V_{t} T_{X}^{1} \theta\right\|_{E} \rightarrow 0 \quad \text { as } t \rightarrow \infty .
$$

It follows from Eq. (4.61), condition (4.62) and the uniqueness of the solution of Eq. (4.66) that $a_{X}^{+}(\theta)=\alpha_{X}^{+}(\theta)$.

Since according to $(4.2),\left(a_{X}^{+}(\theta)\right)(0)=T_{X}\left(\left(a_{1}^{+}(\theta)\right)(0)\right.$, we get by the definition of $\alpha_{X}^{+}(\theta)$ and by the definition $\Omega_{+}(\theta)=\left(a_{1}^{+}(\theta)\right)(0)$ that

$$
T_{X}\left(\left(a_{1}^{+}(\theta)\right)(0)\right)=T_{X}\left(\Omega_{+}(\theta)\right)=\left(D a_{1}^{+}(\theta) \cdot\left(T_{X}^{1} \theta\right)\right)(0)=D \Omega_{+}(\theta) \cdot\left(T_{X}^{1} \theta\right),
$$

$\theta \in \mathcal{O}_{N_{0}+2}$. By continuity it now follows that this inequality is true for $\theta \in \mathcal{O}_{N_{0}+1}$ as $\mathcal{O}_{N_{0}+2}$ is dense in $\mathcal{O}_{N_{0}+1}$. This proves the statement ii) for the case of $\Omega_{+}$. The case of $\Omega_{-}$is so similar that we omit it.

We next consider statement i) in the case of $\mathcal{O}_{N}^{+}$. Since the map $\Omega_{+}: \mathcal{O}_{N^{\prime}}^{+} \rightarrow \mathcal{O}_{N^{\prime}}$ is analytic, so is the map $\Omega_{+}: \mathcal{O}_{N}^{+} \rightarrow \mathcal{O}_{N^{\prime}}$ for $N \geqq N^{\prime}$. Let $\theta \in \mathcal{O}_{N^{\prime}+L}^{+}, L \geqq 1$ and let $X_{1}, \ldots, X_{L} \in \mathfrak{p}$. The map $\theta \mapsto F_{X_{1}, X_{2}, \ldots, X_{L}}(\theta)$, obtained by differentiation of $\Omega_{+}: \mathcal{O}_{N^{\prime}+L}^{+} \rightarrow \mathcal{O}_{N^{\prime}}$ at $\theta$, first in the direction $T_{X_{1}}^{1} \theta$, then in the direction $T_{X_{2}}^{1} \theta, \ldots$, and finally, in the direction $T_{X_{L}}^{1}$ is analytic from $\mathcal{O}_{N^{\prime}+L}^{+}$to $E_{N^{\prime}}$. We prove that in $E$ :

$$
F_{X_{1}, \ldots, X_{L}}(\theta)=T_{Y} \circ A(\theta), \quad Y=X_{1} X_{2} \ldots X_{L}, \quad \theta \in \mathcal{O}_{N^{\prime}+L}, \quad A=\Omega_{+} .
$$

For $L=1$ it follows from Theorem 4.9 that (4.68) is true. Suppose it is true for $L$. Then, for $Y=X_{1}, \ldots, X_{L}$ :

$$
\begin{aligned}
F_{X_{1}, \ldots, X_{L+1}}(\theta) & =\left(D F_{X_{1}, \ldots, X_{L}}(\theta)\right) \cdot\left(T_{X_{L+1}}^{1} \theta\right) \\
& =\left(D\left(T_{Y} \circ A\right)\right)(\theta) \cdot\left(T_{X_{L+1}}^{1} \theta\right)=\left(D T_{Y}(A(\theta))\right) \cdot\left(D A(\theta) \cdot T_{X_{L+1}}^{1} \theta\right) .
\end{aligned}
$$


By statement ii) and definition (1.10) we get:

$$
F_{X_{1}, \ldots, X_{L+1}}(\theta)=\left(D T_{Y}(A(\theta))\right) \cdot T_{X_{L+1}}(A(\theta))=T_{Y X_{L+1}}(A(\theta)),
$$

which proves (4.68) by induction.

We had already chosen $\mathcal{O}_{N_{0}}$ in the proof of Theorem 4.8 such that $\left\|(1-\Delta) \Omega_{+}(\theta)\right\|_{E} \leqq K$, where $K$ is given by Theorem 2.15. If $\theta \in \mathcal{O}_{N}, N \geqq N^{\prime}$, then it follows from (4.68) that $\wp_{N}\left(T\left(\Omega_{+}(\theta)\right)<\infty\right.$. Theorem 2.15 now gives that $\left\|\Omega_{+}(\theta)\right\|_{E_{N}} \leqq \infty$. This proves that $\Omega_{+}: \mathcal{O}_{N}^{+} \rightarrow E_{N}$ is analytic. Furthermore, by the definition of $\mathcal{O}_{N}^{+}$and $\mathcal{O}_{N}^{0}$ it follows that $\Omega_{+}\left(\mathcal{O}_{N}^{+}\right)=\mathcal{O}_{N}^{0}$ as $\Omega_{+}\left(\mathcal{O}_{N^{\prime}}^{+}\right)=\mathcal{O}_{N^{\prime}}^{0}$. This proves together with (4.68) that statement ii) of Theorem 4.9 is true. We omit to prove the remaining points of statement i). Statement iii) is evident as $\Omega_{+}\left(\mathcal{O}_{\infty}^{+}\right)$ $=\bigcap_{N \geqq N^{\prime}} \Omega_{+}\left(\mathcal{O}_{N}^{+}\right)$. This proves the theorem.

We next turn to the proof of the results stated in paragraph one.

Proof of Theorem 1.1. Statement i) is a direct consequence of Theorem 2.3.

Let $\varphi \in C^{\infty}\left(\mathbb{R} \times \mathbb{R}^{2}\right)$ be a solution of Eq. (1.1). The map $g \mapsto \varphi_{g}$ defined by

$$
\varphi_{g}(z)=\varphi\left(\Lambda^{-1}(z-a)\right), \quad g=(a, \Lambda), \quad z=(t, x)
$$

defines an action $\mathscr{P}_{0}$ on solutions of Eq. (1.1), which by the transformation (1.2) defines a continuous action $g \mapsto v_{g}$ of $\mathscr{P}_{0}$ on solutions $v$ of

$$
\frac{d}{d t} v(t)=T_{P_{0}}(v(t)), \quad t \in \mathbb{R}, \quad v(t) \in E_{N_{0}+1} .
$$

Let us define $U_{g}(v(0))=v_{g}(0)$ and we redefine $\mathcal{O}_{N_{0}}^{0}$ as the union of the sets $U_{g}\left(\mathcal{O}_{N_{0}}^{\prime}\right)$ over $g \in \mathscr{P}_{0}$, where $\mathcal{O}_{N_{0}}^{\prime}$ is $\mathcal{O}_{N_{0}}^{0}$ given by Theorem 4.10 and $N_{0}$ is $N^{\prime} . \mathcal{O}_{N}^{0}=E_{N} \cap \mathcal{O}_{N_{0}}^{0}$ is then an open neighbourhood of zero in $E_{N}$ for $N \geqq N_{0}$. It follows from statements i) and iv) of Theorem 4.9 and from statement i) of Theorem 4.10 applied to $\mathcal{O}_{N_{0}}^{\prime}$ that the map $g \mapsto U_{g^{-1}}^{1} U_{g}$ is continuous from $\mathscr{P}_{0}$ into the space of analytic functions from $\mathcal{O}_{N}^{0}$ into $E_{N}$. By construction $U_{g}: \mathcal{O}_{N_{0}}^{0} \rightarrow \mathcal{O}_{N_{0}}^{0}$, so $U_{g^{-1}}^{1} U_{g}$ is an analytic map from $\mathcal{O}_{N}^{0}$ onto $E_{N}$. We have by construction $\frac{d}{d s} U_{g(s)}=T_{X} \circ U_{g(s)}$ for $g(s)=\exp (s X)$ and $X \in \mathfrak{p}$. This proves statements ii) and iii). Statement iv) follows from Corollary 2.16 and by translation by $U_{g}$. This proves Theorem 1.1.

Proof of Theorem 1.2. Since the differential of $U$ is $T$ and the differential of $U^{1}$ is $T^{1}$ it follows from part ii) of Theorem 4.10 , where we have chosen $N_{0} \geqq N^{\prime}$, that for given $g \in \mathscr{P}_{0}$ there exists a neighbourhood $\mathcal{O}_{g}$ of zero in $E_{N_{0}}$ such that $\Omega_{+}^{-1} \circ U_{g}$ $=U_{g}^{1} \circ \Omega_{+}^{-1}$ in $\mathcal{O}_{g}$. By analytic extension this equality is true on $\mathcal{O}_{N_{0}}^{0}$, which also proves that $\Omega_{+}^{-1}$ is defined on $\mathcal{O}_{N_{0}}^{0}$. In fact, this follows from the construction of $\mathcal{O}_{N_{0}}^{0}$ and the uniqueness of the solutions of the scattering problem in statement ii) of Theorem 4.9. We define $\mathcal{O}_{N_{0}}^{+}$by $\mathcal{O}_{N_{0}}^{+}=\Omega_{+}^{-1}\left(\mathcal{O}_{N_{0}}^{0}\right)$. Similarly we define $\Omega_{-}$and $\mathcal{O}_{N_{0}}^{-}$. It follows from statement ii) of Theorem 4.9 that $\Omega_{\varepsilon}: \mathcal{O}_{N}^{\varepsilon} \rightarrow \mathcal{O}_{N}^{0}$ is analytic as well as its inverse. This proves statements i) and ii) of the theorem. Statement iii) follows from statement ii) of Theorem 4.9 and by the construction of $\mathcal{O}_{N_{0}}^{0}$. This proves Theorem 1.2.

Proof of Theorem 1.3 and Theorem 1.4. Theorem 1.3 is a particular case of Theorem 1.2. Let $\theta \in \mathcal{O}_{\infty}^{0}$ and let its image under the transformation (1.3) be $\varphi_{0}, \dot{\varphi}_{0}$. After a change of $\varphi_{0}, \dot{\varphi}_{0}$ on a set of measure zero, $\varphi_{0}, \dot{\varphi}_{0} \in \mathscr{S}\left(\mathbb{R}^{2}\right)$. The map 
$\theta \mapsto\left(\varphi_{0}, \dot{\varphi}_{0}\right)$ so defined is continuous and invertible. Define $\mathcal{O}^{\prime} \subset \mathscr{S}\left(\mathbb{R}^{2}\right) \times \mathscr{S}\left(\mathbb{R}^{2}\right)$ as the image of this map. $\mathcal{O}^{\prime}$ is an open neighbourhood of zero in $\mathscr{S}\left(\mathbb{R}^{2}\right) \times \mathscr{S}\left(\mathbb{R}^{2}\right)$. There exist two neighbourhoods $\mathcal{O}, \dot{\mathcal{O}}$ of zero in $\mathscr{S}\left(\mathbb{R}^{2}\right)$ such that $\mathcal{O} \times \mathcal{O} \subset \mathcal{O}^{\prime}$. For $\left(\varphi_{0}, \dot{\varphi}_{0}\right) \in \mathcal{O} \times \dot{\mathcal{O}}$ it follows from Theorem 1.3 that there exists a $C^{1}$ solution $t \mapsto v(t) \in E_{N}, t \in \mathbb{R}$, for each $N \geqq 0$ of the equation $\frac{d}{d t} v(t)=T_{P_{0}}(v(t))$. Differentiation in $t$ of this equation shows that the map $t \mapsto v(t) \in E_{N}, N \geqq 0$ is $C^{\infty}$. Hence by transformation (1.3) we obtain (after a change on a set of measure zero) a solution $\varphi \in C^{\infty}\left(\mathbb{R} \times \mathbb{R}^{2}\right)$ of Eq. (1.1) which satisfies the given initial conditions.

\section{Appendix}

As it was already mentioned in the introduction, the methods developed in this paper also give existence of global solutions for time $t \in \mathbb{R}$ and asymptotic completeness for Eq. (1.1) when it is not covariant under the action of the Poincare group. In this case the inverse of the wave operator only linearizes the nonlinear representation of the space-time translation group $\mathbb{R}^{n+1}$. From the point of view of fundamental physics, the Poincare covariant case is certainly more natural than the $\mathbb{R}^{n+1}$ covariant case. Moreover, the stronger results in the Poincare covariant case are more difficult to prove, although the hypothesis in the $\mathbb{R}^{n+1}$ covariant case are weaker. But, as the results for the $\mathbb{R}^{n+1}$ covariant case follow, without any essential change in the proof of this paper and as the $\mathbb{R}^{n+1}$ covariant case could be interesting for readers mainly focused on partial differential equations, we give an outline of the proof when Eq. (1.1) is not necessarily covariant under the Poincaré group $\mathscr{P}_{0}$.

Suppose that $P$ is such that Eq. (1.1) is not Poincare covariant. Even in this case Eq. (1.1) is $\mathbb{R}^{n+1}$ covariant. Let first $P$ be an analytic function. We define the Lie algebra representation $T_{X}^{1}, X \in \mathfrak{p}$ on $E_{\infty}$ as in (1.6) and the Hilbert spaces $E_{i}, i \in \mathbb{N}$ as in (1.7). $T_{X}=T_{X}^{1}+\widetilde{T}_{X}, X \in \mathfrak{p}$ is defined by formulas (1.8) and (1.9). If $X, Y \in \mathbb{R}^{n+1}$, the radical of $\mathfrak{p}$, then $\left[T_{X}, T_{Y}\right]=0$, where $\left[T_{X}, T_{Y}\right]=D T_{X} \cdot T_{Y}-D T_{Y} \cdot T_{X}$. Hence $T_{X}$ is a nonlinear representation of $\mathbb{R}^{n+1}$. But, according to the hypothesis that Eq. (1.1) is not Poincaré covariant, $\left[T_{X}, T_{Y}\right] \neq T_{[X, Y]}$, for some $X, Y \in \mathfrak{p}$, so $T_{X}$ is no more a nonlinear representation of $\mathfrak{p}$. The linear map $X \mapsto T_{X}, X \in \mathfrak{p}$ is extended to the tensor algebra $t(\mathfrak{p})$ by formula (1.10): $T_{Y X}=D T_{Y} \cdot T_{X}, X \in \mathfrak{p}, Y \in t(\mathfrak{p}), T_{1}=I$. Here it is not possible to pass to the quotient space to obtain a map from $U(\mathfrak{p})$ to the space of polynomials on $E_{\infty}$. Now we consider that $Y(t) \in t(\mathfrak{p})$. Then (1.12) to (1.15) are true, but there is no chance to find a solution $A$ of (1.16) for all $X \in \mathfrak{p}$. However, (1.16) turn out to have a solution for any $X \in \mathbb{R}^{n+1}$, the radical of $\mathfrak{p}$. Formulas (1.17) to (1.19) still hold. To obtain Theorem 2.15, we change definition (2.38) of $\wp_{N}$ as

$$
\wp_{N}(a)=\left(\sum_{Y}\left\|a_{Y}\right\|_{E}^{2}\right)^{1 / 2},
$$

where the sum is taken over all elements of degree at most $N$ belonging to the basis of $t(\mathfrak{p})$ built from the standard basis of $\mathfrak{p}$ by tensor products.

Theorem 3.9 is obtained by a direct calculation of the commutator $T_{Y}^{1} A^{2}-A^{2} S_{Y}, Y \in t(\mathfrak{p})$, where $S_{X}=T_{X}^{1} \otimes I+I \otimes T_{X}^{1}$. Theorem 1.1 has the following analog: 
Theorem 1.1'. For $n \geqq 2$ there exists $N_{0} \geqq 0$ and a neighbourhood $\mathcal{O}_{N_{0}}^{0}$ of zero in $E_{N_{0}}$ such that, if $\mathcal{O}_{N}^{0}=E_{N} \cap \mathcal{O}_{N_{0}}^{0}$ for $N \geqq N_{0}$ and $\mathcal{O}_{\infty}^{0}=E_{\infty} \cap \mathcal{O}_{N_{0}}^{0}$, then:

i) $T_{X}, X \in \mathbb{R}^{n+1}$ defined by (1.8) is a nonlinear analytic Lie-algebra representation on $\mathcal{O}_{\infty}^{0}$. For $X \in \mathbb{R}^{n+1} \in \operatorname{so}(n, 1)=\mathfrak{p}, T_{X}: \mathcal{O}_{N+1}^{0} \rightarrow E_{N}$ and $\widetilde{T}_{X}: \mathcal{O}_{N}^{0} \rightarrow E_{N}$ are analytic maps.

ii) $T_{X}, X \in \mathbb{R}^{n+1}$ is the differential of a unique global nonlinear analytic group representation $U$ of $\mathbb{R}^{n+1}$, i.e. $U_{g}(\theta) \in \mathcal{O}_{N_{0}}^{0}$ for $g \in \mathbb{R}^{n+1}, \theta \in \mathcal{O}_{N_{0}}^{0}$ and the map $g \mapsto U_{g^{-1}}^{1} U_{g}$ is continuous from $\mathbb{R}^{n+1}$ into the space $\mathscr{H}\left(\mathcal{O}_{N_{0}}^{0}, E_{N_{0}}\right)$, where $U^{1}$ is the linear part of $U$.

iii) For $N \geqq N_{0}$, the map $g \mapsto U_{g^{-1}}^{1} U_{g}$ is continuous from $\mathbb{R}^{n+1}$ into the space $\mathscr{H}\left(\mathcal{O}_{N}^{0}, E_{N}\right)$.

We note that the counterpart of statement iv) of Theorem 1.1 is no longer true for the $\mathbb{R}^{n+1}$ covariant case. We can only conclude that $\mathcal{O}_{\infty}^{0}$ is a set of differentiable vectors for $g \mapsto U_{g}, g \in \mathbb{R}^{n+1}$, but not the set of all differentiable vectors.

Theorem 1.2, stating the existence of wave operators, still holds if $\mathscr{P}_{0}$ is replaced by $\mathbb{R}^{n+1}$. Theorem 1.3 and Theorem 1.4, stating the existence of global solutions for $t \in \mathbb{R}$, then remain true as they are formulated. They are as a matter of fact particular cases of Theorem 1.1' and Theorem 1.2. We also note that Theorem 1.4 can be formulated with Hilbert space neighbourhoods of initial conditions $\mathcal{O}_{N+1} \times \dot{\mathcal{O}}_{N+1}, N \geqq N_{0}$ being the image of $\mathcal{O}_{N+1}^{0}$ under the transformation (1.3) and solution $t \mapsto \varphi(t) \in \mathcal{O}_{N}, t \in \mathbb{R}$. This follows immediately from Theorem 1.3. Theorem 1.4 as it is formulated with $\mathcal{O} \times \dot{\mathcal{O}}$, is as a matter of fact more difficult, because one has to prove that the intersection of the family $\left\{\mathcal{O}_{N} \times \dot{\mathcal{O}}_{N}\right\}_{N \geqq N_{0}}$ is a neighbourhood in $\mathscr{S}\left(\mathbb{R}^{3}\right) \times \mathscr{S}\left(\mathbb{R}^{3}\right)$. The sets $\mathcal{O}_{N} \times \dot{\mathcal{O}}_{N}$ are neighbourhoods in weighted energy spaces.

Let us finally relax the hypothesis of analyticity of $P$ in Eq. (1.1) and only require that $P$ is $C^{\infty}$. Then the above modified results, i.e., Theorem 1.1', Theorem 1.2 with $\mathbb{R}^{n+1}$ instead of $\mathscr{P}_{0}$, and Theorems 1.3 and 1.4 , are still true if, in their formulation, analytic is systematically replaced by $C^{\infty}$.

To sum up, as it was noted in the introduction, we have proved the existence of global solutions for $t \in \mathbb{R}$, the existence of $C^{\infty}$ invertible wave operators and asymptotic completeness for the massive Klein-Gordon equation (1.1) with the most general $C^{\infty}$ non-linearity $P$ on a set of small initial conditions, being a neighbourhood of zero in a weighted energy space.

Acknowledgements. The authors wish to thank Professor M. Flato for stimulating discussions. We would like also to thank the referee of this paper for pointing out the existence of the reference: V. Georgiev, P. Popivanov: Commun. Part. Diff. Eq. 16 (6, 7), 941-995 (1991). Existence of global solutions for the particular case of Eq. (1.1) with

$$
P\left(\varphi, \frac{\partial}{\partial t} \varphi, \nabla \varphi\right)=\varphi^{2}+\left(\frac{\partial}{\partial} \varphi\right)^{2}-\left(\partial_{1} \varphi\right)^{2}-\left(\partial_{2} \varphi\right)^{2}
$$

is studied in that reference for $n=2$ and for smooth initial conditions with compact support.

\section{References}

1. Flato, M., Pinczon, G., Simon, J.C.H.: Non-linear representations of Lie groups. Ann. Sci. Ec. Norm. Super. 10, 405-418 (1977)

2. Hörmander, L.: Remarks on the Klein-Gordon equation. Journée Equations aux dérivées partielles. Saint-Jean de Monts 1987, Soc. Math. France (1987) 
3. Hörmander, L.: The analysis of linear partial differential operators, Vol. III. Berlin, Heidelberg, New York: Springer 1985

4. Klainerman, S.: Global existence of small amplitude solutions to nonlinear Klein-Gordon equations in four space-time dimensions. Commun. Pure Appl. Math. 38, 631-641 (1985)

5. Shatah, J.: Normal forms and quadratic non-linear Klein-Gordon equations. Commun. Pure Appl. Math. 38, 685-696 (1985)

6. Simon, J.C.H.: A wave operator for a non-linear Klein-Gordon equation. Lett. Math. Phys. 7, 387-398 (1983)

7. Simon, J.C.H., Taflin, E.: Wave operators and analytic solutions for systems of non-linear Klein-Gordon equations and non-linear Schrödinger equations. Commun. Math. Phys. 99, 541-562 (1985)

8. Warner, G.: Harmonic analysis on semi-simple Lie groups I, Vol. 188. Berlin, Heidelberg, New York: Springer 1972

Communicated by A. Jaffe 